\title{
Measuring the Market Risk of Freight Rates: A Forecast Combination Approach
}

\author{
Christos Argyropoulos* \\ Kent Business School, UK \\ Ekaterini Panopoulou ${ }^{\dagger}$ \\ Kent Business School, UK \& University of Piraeus, Greece
}

May 10, 2015

\begin{abstract}
This paper aims at contributing to the literature in three ways: First, we re-evaluate the performance of popular Value-at-Risk (VaR) estimation methods on freight rates amid the adverse economic consequences of the recent financial and sovereign debt crisis. Second, we provide a detailed and extensive backtesting and evaluation methodology. Last, we propose a forecast combination approach for estimating VaR. Our findings suggest that our combination methods produce more accurate estimates for all the sectors under scrutiny, while in some cases they may be viewed as conservative since they tend to overestimate nominal VaR.
\end{abstract}

Keywords: Backtesting; Combination Forecasts; Volatility Forecasts; Freight Rates; Performance Evaluation; Value-at-Risk;

Acknowledgements: We thank the participants of the 7th International Conference on Computational and Financial Econometrics and the 3rd National Conference of the Financial Engineering and Banking Society for their helpful comments and suggestions. The usual disclaimer applies.

* Argyropoulos Christos, Kent Business School, University of Kent, Canterbury CT2 7PE, United Kingdom. E-mail: c.argyropoulos@kent.ac.uk.

${ }^{\dagger}$ Correspondence to: Ekaterini Panopoulou, Kent Business School, University of Kent, Canterbury CT2 7PE, United Kingdom, Tel.: 00441227 824469, Email: A.Panopoulou@kent.ac.uk. 


\section{Introduction}

Freight rate risk has always been one of the most important risk factors of the shipping industry mainly because it affects its primary source of income. In addition, the uprising interest of participants in the shipping industry such as shipping companies, shipping hedge funds and shipping banks makes the accurate measurement of freight risk a procedure of high importance. However the intrinsic characteristics of the shipping industry hinder the accurate measurement of market risk. The cyclicality of maritime economy alongside the shipping markets mechanics create a complex profile for the freight rates and their volatility. ${ }^{1}$ For instance Alizadeh and Nomikos (2011) argue that volatility dynamics vary depending on shipping market conditions. In the same vein, Alizadeh et al. (2014) implemented a regime switching multivariate approach in order to capture the volatility dynamics and possible correlations of spot and FFA prices. Consequently, the necessity of producing accurate estimates of freight risk is essential to freight market participants as it enables them to enhance their ability of sound strategic, investment and hedging decisions. This paper addresses the subject of freight rate risk measurement via Value at Risk (VaR) and forecast combination methodologies while focusing on detailed performance evaluation.

Despite the plethora of related research in the financial sector, the measurement of the market risk of freight rates has not been a popular subject. To the best of our knowledge the most recent contributions in the field of freight rate risk adopt the VaR methodology in order to measure the market risk of the dry bulk and tanker freight market. Specifically, Angelidis and Skiadopoulos (2008) applied a variety of parametric, non-parametric and hybrid methods in order to measure the market risk mainly for the dry bulk sector. Their findings suggest that in almost all cases the simplest non-parametric methods produce accurate results. On the other hand, Kavussanos and Dimitrakopoulos (2011) dealt with the selection of the appropriate freight rate risk model by applying a similar VaR methodology solely for the tanker sector. The authors find that parametric methods are more suitable for this sector. More recently Abouarghoub et al.(2014) utilized a two state Markov-Switching distinctive conditional variance model in order to improve the tanker sector VaR forecasts. Their results suggest that a regime switching approach can capture more precisely the tanker sector volatility dynamics, thus providing better VaR forecasts.

Calculating precisely freight rate risk is of utmost importance for at least three reasons. First, market participants can develop hedging schemes more effectively and efficiently when they are aware of the risk they are exposed to. Simple risk metrics, like volatility have not been proven adequate for this market due to deviation of normality, complexity, cyclicality and the existence of jumps during extreme events (see e.g. Kavussanos and Visvikis, 2004, Kavussanos and Dimitrakopoulos, 2011, Alizadeh and Nomikos, 2011). Second, during the last decade, the shipping freight market has transformed from a service market, where freight rate was the cost of transporting raw materials by sea to a market where freight rate is seen as an investment like any other asset or commodity (see e.g. Nomikos et al., 2013). Market participants now

\footnotetext{
${ }^{1}$ For an elaborate discussion on freight rate characteristics, see Stopford (2009), Alizadeh and Nomikos (2009).
} 
include investment banks and hedge funds, who are interested in quantifying the risk profile of this class having realized the potential benefit of this alternative asset class for both speculation and diversification. Alizadeh (2013) documents a positive contemporaneous relationship between trading volume, which has increased rapidly in the recent years, and volatility in the shipping forward freight market. Finally, VaR provides a mean of setting margin requirements in the freight exchange derivatives market, which is expanding fast. Given that forward freight agreements and freight options are employed to hedge freight rate risk and that trading of these derivatives can be done through an organized exchange, margin determination is a prerequisite. With the elimination of credit risk, margins reflect market liquidity and volatility of the underlying spot freight rates. ${ }^{2}$

In this paper, we contribute to the literature on freight rate risk forecasting in the following dimensions. First, we re-evaluate the performance of popular VaR estimation methods amid the adverse economic consequences of the recent financial and sovereign debt crisis. Second, we provide a detailed and extensive backtesting methodology in order to identify possible weaknesses associated with the standard backtesting criteria. Finally, we propose a forecast combination approach in estimating VaR, which provides more accurate VaR estimates while reducing the cost in time and resources.

More in detail, we calculate the daily $5 \%$ and $1 \%$ VaR of a long position comprising of the most important Baltic Exchange indices by applying a wide range of estimation methods. The aggregate freight rate indices employed are averages of individual route or time chartered indices and can be thought of as portfolios of freight rate positions covering large fleets of vessels. On the other hand, individual route indices are more relevant for market risk exposures of smaller companies, since these companies employ vessels in one of these routes which also serve as the underlying assets of freight rate derivatives (see Kavussanos and Dimitrakopoulos, 2011). ${ }^{3}$ To account for both types of risk exposure, we complement our analysis with the most actively utilized individual routes of both the dry bulk and the tanker sector. Detailing our methodology, we apply parametric, non-parametric, hybrid and a variety of combination methods on the logarithmic returns of the indices at hand and employ an evaluation sample that includes both blooming periods and the crises periods of 2007-2009. Given the plethora of VaR estimation methods, we go one step further and investigate whether combinations of VaR forecasts can lead to gains such as: diversification gains, robustness to model mispecification and structural breaks and bias correction of individual VaRs (see e.g. Timmerman, 2006 and Halbleid and Pohlmeier, 2012). To this end, we employ the mean, median and trimmed means (two versions) of individual VaR estimates aiming at producing a superior forecast. In order to

\footnotetext{
${ }^{2}$ Predictability in the underlying freight spot rate does not imply predictability of the corresponding derivative contract, since the standard cost-of-carry relationship for financial futures does not hold for the freight ones. This is because the underlying asset is not tradable, and hence the pricing by arbitrage argument cannot be applied. A series of papers have tested the unbiasedness hypothesis of the market, i.e. whether the freight rate market is efficient. Results are mixed mainly due to market segmentation (see Kavussanos and Visvikis, 2006, for a review). A more recent contribution by Goulas and Skiadopoulos (2012) points to the inefficiency of the International Maritime Exchange (IMAREX) freight futures markets over the daily horizon. The futures trading strategies based on the formed daily forecasts the authors develop yield a positive, economically significant risk premium.

${ }^{3}$ We thank an anonymous referee for bringing this issue to our attention.
} 
conduct a more reliable and in depth evaluation, we implement a battery of newly developed backtesting criteria, namely the Engle and Manganelli (2004) quantile regression approach, the Christoffersen and Pelletier (2004) duration approach and the Colletaz et al. (2013) test along with the standard Christoffersen (1998) approach. More importantly, we complement statistical evaluation with the performance evaluation methodology proposed by Sener et al. (2012) in order to rank the implemented methods.

To anticipate our key results, we find evidence that the combination methods constitute a strong alternative to the plethora of individual methods. Specifically we find that at the $5 \%$ VaR level, combination methods provide a globally accepted method which in every case under scrutiny produces equal or superior results to the highest ranked individual method. Quite importantly, our findings are more pronounced at the $1 \% \mathrm{VaR}$ level as we find that in the majority of cases combination methods are superior to the individual ones. We anticipate the empirical findings of the paper to be useful to a wider portion of market participants who undertake risks in markets that exhibit similar characteristics with freight rate markets such as energy and commodity markets.

The remainder of this paper is organized as follows; Sections 2 and 3 present the theoretical framework and the methodologies that we employ in order to compute and evaluate the VaR estimates. Section 4 presents the data and the empirical findings while Section 5 presents the related findings for individual route indices. Section 6 concludes the paper.

\section{VaR methodology}

\subsection{Value at Risk}

Materialization of risk can be an extremely challenging procedure as every case presents different needs and characteristics. However, there is a large arsenal of tools available for each case and it is up to the risk manager to pick the appropriate one. The core of these tools are the employed risk measures. There are three basic categories of risk measures; sensitivity measures, volatility measures and finally downside risk measures. ${ }^{4}$ Downside risk measures are thought to be the most comprehensive category as they combine both sensitivity and volatility measures with the adverse effect of uncertainty. A typical example of a downside risk measure is Value at Risk (VaR) which can be described schematically as the maximum potential deviation for a given significance level over a given time horizon. More in detail, let $p_{t}$ be the price of the asset at the end of the day $t$ and $r_{t}=\log \left(\frac{p_{t}}{p_{t-1}}\right)$ the daily $\log$ returns. For a long position, VaR is defined as the expected maximum loss over a specific horizon at a certain confidence level. Mathematically, it can be defined at a $q \%$ confidence level as follows:

$$
P\left(r_{t} \leq-\operatorname{VaR}(q)\right)=q
$$

\footnotetext{
${ }^{4}$ For an elaborate discussion, see Bessis (2002).
} 


\subsection{Value at Risk methods}

In our analysis, we implement a variety of methods which can be divided into four categories; parametric, non-parametric, hybrid and combination methods in order to estimate VaR. The first two categories can be characterized as the genuine individual methods that introduce different approaches based on specific concepts. The last two categories consist of methods that combine the components or the advantages of the individual methods in order to produce better results.

\subsubsection{Parametric methods}

The parametric approach assumes that the series of logarithmic returns is a stochastic process which is described by the following set of equations:

$$
\begin{gathered}
r_{t}=\mu_{t}+\varepsilon_{t}, \varepsilon_{t}=z_{t} \sigma_{t} \\
\left.z_{t} \sim f\left(E\left(z_{t}\right)=0\right), V\left(z_{t}\right)=1, \theta\right) \\
\sigma_{t}^{2}=g\left(I_{t-1}, w\right)
\end{gathered}
$$

where $\mu_{t}$ is the mean return, $\varepsilon_{t}$ is the time varying standard deviation error, $f($,$) denotes the$ probability function of $z_{t}$ given the parameter $\theta$ and $g($,$) denotes the variance process given the$ parameter $w$ and the past information $I_{t-1}$. Based on the above process, we can estimate VaR as follows:

$$
\widehat{\operatorname{VaR}_{t}}(q)=\widehat{\mu}_{t}+F_{q}^{-1}(z, \theta) \widehat{\sigma_{t}}
$$

where $\widehat{\mu_{t}}$ is the mean return estimate, $F_{q}^{-1}$ is the quantile of the standardized theoretical distribution given the estimated parameter $\theta$, and $\widehat{\sigma_{t}}$ is the estimate of the standard deviation given the estimated parameter $w$. If the series of returns present autocorrelation, while stationary, the AutoRegressive Moving Average (ARMA) model approach is suitable for the mean estimation. The $\operatorname{ARMA}(P, Q)$ process is given by the following equation:

$$
\mu_{t}=\varphi_{0}+\sum_{i=1}^{P} \varphi_{i} r_{t-i}+\sum_{j=1}^{Q} \psi_{j} \varepsilon_{t-j}
$$

where $\sum_{i=1}^{P} \varphi_{i} r_{t-i}$ denotes the dependence with lagged observations of returns and $\sum_{j=1}^{Q} \psi_{j} \varepsilon_{t-j}$ denotes the dependence of returns with lagged errors. To model volatility, we employ models that belong to the Generalized AutoRegressive Conditional Heteroskedasticity (GARCH) family. Specifically, we employ the AutoRegressive Conditional Heteroskedastic (ARCH) model proposed by Engle (1982) which can be considered a special case of the GARCH models proposed by Bollerslev (1986). A $\operatorname{GARCH}(P, Q)$ model is given by the following equation:

$$
\sigma_{t}^{2}=\alpha_{0}+\sum_{i=1}^{P} \alpha_{i} \varepsilon_{t-i}^{2}+\sum_{j=1}^{Q} b_{j} \sigma_{t-j}^{2}
$$




$$
\alpha_{0}>0, \alpha_{i} \geq 0, b_{j} \geq 0 \text { and } \sum_{i=1}^{\max (P, Q)}\left(\alpha_{i}+b_{i}\right)<1
$$

while ARCH models can be derived from equation (6) for $Q=0$. While the above models can sufficiently capture the volatility of returns, they cannot discriminate between negative or positive prior returns. In order to capture the potential leverage effect we employ the Exponential GARCH (EGARCH) models proposed by Nelson(1991). The $\operatorname{EGARCH}(P, Q)$ model is described by the following equation:

$$
\begin{gathered}
\log \left(\sigma_{t}^{2}\right)=\alpha_{0}+\sum_{i=1}^{P}\left(\alpha_{i}\left|\frac{\varepsilon_{t-i}}{\sigma_{t-i}}\right|+\gamma_{i} \frac{\varepsilon_{t-i}}{\sigma_{t-i}}\right)+\sum_{j=1}^{Q} b_{j} \log \left(\sigma_{t-j}^{2}\right) \\
\alpha_{0}>0, \alpha_{i} \geq 0, b_{j} \geq 0 \text { and } \sum_{i=1}^{\max (P, Q)}\left(\alpha_{i}+b_{i}\right)<1
\end{gathered}
$$

In order to estimate the parameters of models (5)-(7), we employ both the Normal and Student-t distributions as the theoretical return distribution.

Finally, we follow the Extreme Value Theory (EVT) approach in order to simulate the tail of the empirical returns distribution and avoid the implementation of symmetrical distributions that may not take account of the unique properties of the empirical distribution of returns. Specifically, we use the Hill estimator in order to attain the parameter of the generalized Pareto distribution while setting as a threshold the $5 \%$ quantile of the sample of returns. The mean return is calculated as described in equation (5), while the standard deviation is estimated by a GARCH(1,1)-t model. The EVT-VaR is estimated as follows;

$$
\begin{gathered}
\widehat{\operatorname{VaR}_{t}}(q)=\widehat{\mu_{t}}+\widehat{\sigma_{t}} u\left(\frac{q}{T_{u} / T}\right)^{(-\xi)} \\
\xi=\frac{1}{T_{u}} \sum_{i=1}^{T_{u}} \ln \left(\frac{y_{i}}{u}\right), y_{i}=r_{i}-u
\end{gathered}
$$

where $\left(\frac{q}{T_{u} / T}\right)^{(-\xi)}$ is the $q$ quantile of the generalized Pareto distribution, $u$ denotes the threshold and finally $T$ and $T_{u}$ are the number of sample observations included in the evaluation sample and the calculation of the Hill estimator (Equation 9) respectively.

\subsubsection{Variance Covariance and Riskmetrics Methods}

While Variance Covariance and Riskmetrics can be thought as parametric methods, they have by definition some unique characteristics that hinder their classification as pure parametric. The Variance Covariance method utilizes the assumptions of homoscedasticity and normality of returns and uses the sample standard deviation as a proxy for the volatility of returns. The VaR estimates are produced as follows:

$$
\widehat{\operatorname{VaR}_{t}}(q)=F_{q}^{-1}(z, \theta) \widehat{\sigma}
$$


where $F_{q}^{-1}(z, \theta)$ denotes the $q$ quantile of the normal distribution and $\widehat{\sigma}$ is the sample standard deviation.

On the other hand, Riskmetrics assumes normality of returns but rejects the homoscedasticity. In addition to that, returns depend only on the past volatility as captured by an Integrated GARCH (IGARCH) model with fixed parameters. The VaR estimates are produced by the following set of equations

$$
\begin{gathered}
r_{t}=\varepsilon_{t}, \varepsilon_{t}=z_{t} \sigma_{t} \\
\sigma_{t}^{2}=0.04 \varepsilon_{t-1}^{2}+0.96 \sigma_{t-1}^{2} \\
\widehat{\operatorname{VaR}}(q)=F_{q}^{-1}(z, \theta) \widehat{\sigma_{t}}
\end{gathered}
$$

where $F_{q}^{-1}(z, \theta)$ denotes the $q$ quantile of the normal distribution and $\widehat{\sigma}_{t}$ is the estimated conditional standard deviation.

\subsubsection{Non Parametric Methods- Historical Simulation}

In contrast to the parametric methods, the non-parametric ones calculate VaR using exclusively the empirical distribution of returns. The idea underlying the non-parametric processes is that of the repetition of past losses. Based on this dogma, VaR is calculated as:

$$
\widehat{\operatorname{VaR}_{t}}(q)=F_{q}^{-1}\left(\left\{r_{i}\right\}_{i=1}^{t-1}\right)
$$

where $F_{q}^{-1}$ denotes the $q$ quantile of the sample of returns. The main advantage of this approach, except for its simplicity, is the use of the actual fat tails and skewness of the empirical distribution instead of the theoretical one. In our study, we employ three versions of Historical Simulation estimates based on data samples of 250, 500 and $\mathrm{T}$ observations corresponding to a window of one year, two years and the whole sample.

\subsubsection{Hybrid Methods}

The hybrid methods have been developed in order to address some disadvantages associated with the individual methods. Such disadvantages are the inability of the parametric methods to incorporate the properties of the empirical returns distribution and the Historical Simulation's inability to capture a sudden change in the volatility. In this paper we employ the Filtered Historical Simulation proposed by Barone-Adesi et al. (1999) and a Monte Carlo Simulation method. Filtered Historical Simulation was proposed as an improvement of the Historical Simulation. It employs the mean and standard deviation procedure of the parametric methods (Equations (5)-(7)) in conjunction with the properties of the empirical distribution of returns. Essentially this method can be viewed as a parametric method without the theoretical distribution hypothesis. In this paper, we model returns via an ARMA-GARCH(1,1)- $t$ model and the VaR is calculated as follows:

$$
\widehat{\operatorname{VaR}_{t}}(q)=\widehat{\mu_{t}}+F_{q}^{-1}\left(\left\{z_{i}\right\}_{i=1}^{t-1}\right) \widehat{\sigma_{t}}
$$


where $F_{q}^{-1}\left(\left\{z_{i}\right\}_{i=1}^{t-1}\right)$ denotes the $q$ quantile of the standardized residuals. The main advantage of this approach is the use of the empirical distribution properties embedded in the standardized residuals, by which the properties of the empirical distribution are depicted on the calculation of VaR. Monte Carlo simulation estimates VaR as a quantile of a simulated empirical distribution of returns. Specifically an ARMA-GARCH(1,1) - $t$ model is employed in order to produce 10000 daily pseudo returns and VaR is produced as the $q t h$ quantile of these simulated returns.

\subsection{Combination Methods}

The methods described so far have been designed to take into account certain empirical properties which in turn lend to each method specific characteristics and weaknesses. So the question is whether appropriate combination of these methods can eliminate inefficiencies and ultimately produce improved results compared to the individual methods. Timmermann (2006) suggests that combination estimators display superior performance because they combine the information embedded into each individual estimator while they are less sensitive to structural breaks and mispecification of individual methods. Profits from combination forecasts also arise from diversification gains and correction for bias of individual forecasts.

So far the macroeconomic and financial literature suggests that the combination of individual forecasts produce superior results (see for example Stock and Watson, 2004; Huang and Lee, 2010; Rapach et al., 2010). In the field of combination forecasts and especially volatility forecasts, the most recent contributors (Becker and Clements, 2008; Patton and Sheppard, 2009; Andreou et al., 2012) have addressed the issue of the performance of volatility combinations concluding that in most of the cases the combination estimators surpass the individual methods. Specifically Becker and Clements (2008) investigate the performance of the S\&P 500 Implied Volatility forecasts relative to model based forecasts and their combinations. Their results suggest that the combinations of model based forecasts are superior. In the same vein, Patton and Sheppard (2009) investigate the performance of Realized Volatility combination estimators relative to the individual ones and find that the simplest combination schemes produce superior results in most of the cases. Finally, Andreou et al. (2012) address the issue of model uncertainty in volatility by using a comprehensive model space and investigate whether a combination framework can improve volatility forecasts. More precisely, the authors consider the simple AutoRegressive models of Realized Volatility (AR-RV), the Heterogeneous Autoregressive model of Realized Volatility (HAR-RV) (Corsi, 2009) and the Leverage HAR-RV (LHAR-RV)(Corsi and Reno, 2009) in addition to GARCH-type volatility models and nonparametric models of volatility using rolling volatility estimators. Their results suggest that forecast combinations based on an homogeneous robust loss function significantly outperform simple forecast combination methods, especially during the period of the recent financial crisis.

Risk management practices during the recent financial crisis is the focus of two recent papers closely related to our approach. ${ }^{5}$ McAleer et al. (2013) suggest using a combination of VaR forecasts to obtain a crisis-robust risk management strategy for a variety of international

\footnotetext{
${ }^{5}$ We thank the editor for bringing these papers to our attention.
} 
stock market indices. The authors develop VaR forecasts using combinations of the forecasts of individual VaR models, namely the $r$ th percentiles of the VaR forecasts of a set of univariate conditional volatility models. Their findings suggest that the median of the point VaR forecasts is a risk management strategy that is a superior risk management strategy compared to strategies based on single and composite model alternatives. Relaxing the assumption of deterministic weights on individual forecasts, Halbleib and Pohlmeier (2012) propose a new methodology of computing VaRs based on the principle of optimal combination that accurately and robustly forecasts losses during periods of high risk. They consider two ways of computing optimal weights; first by minimizing the distance between the population and empirical moments derived from Basel II rules and other VaR evaluation techniques and second by employing simple quantile regressions on stand-alone VaR forecasts. Their combination forecasts exhibit a stable in-sample and out-of-sample performance across both calm and turbulent evaluation periods.

Our approach is more related to McAleer et al. (2013). In the same spirit, we aim at dealing with the adverse effects of the recent financial crisis on the potential mispecification and inaccuracy of individual VaR methods by appropriately combining them. More precisely, we employ the mean, median and trimmed mean combination schemes of a plethora of individual VaR forecasts. From an empirical point of view, the risk associated with the selection of a specific individual model is eliminated, volatility of forecasts is reduced and structural instability is minimized. Moreover, our approach does not suffer from estimation error since weights are not estimated and is relevant to a wider audience due to its computational tractability.

Specifically, let us denote with $\widehat{V a R_{i t}}, i=1,2, \ldots, n$ the $n$ individual VaR methods. Then the mean, trimmed mean and median combining schemes for the VaR forecasts are the following:

$$
\begin{gathered}
\widehat{V a R}_{\text {Mean }, t}(q)=\frac{\sum_{i=1}^{n} \widehat{V a R_{i t}}}{n} \\
\widehat{V a R}_{\text {Trimmed } 1, t}(q)=\frac{\sum_{i^{\prime}=1}^{n-1} \widehat{V a R_{i^{\prime}} t}}{n-2} \\
\widehat{V a R}_{\text {Trimmed } 2, t}(q)=\frac{\sum_{i^{\prime}=2}^{n-2} \widehat{V a R_{i^{\prime}} t}}{n-4} \\
\widehat{V a R}_{\text {Median }, t}(q)=\operatorname{Median}\left(\left\{\widehat{\operatorname{VaR} i^{\prime} t}\right\}_{i^{\prime}=1}^{n}\right)
\end{gathered}
$$

where $\widehat{V a R_{i^{\prime}} t}$ signifies the sorted, in an ascending order, individual estimates.

\section{Evaluation Framework}

In order to evaluate the implemented methodology and compare the individual methods with the combination methods, we use a two-step evaluation framework. First, we examine in detail the statistical accuracy of each method by applying a battery of backtesting criteria. Secondly, we evaluate the forecasting performance by utilizing the newly proposed methodology of Sener et al. (2012) in order to rank the VaR methods. These steps are considered independently while the final results are produced by ranking only the statistically accepted methods. 


\subsection{Statistical Evaluation}

To statistically evaluate the employed VaR methods we use three different approaches plus an additional test. First we employ the approach proposed by Christoffersen (1998) which consists of three criteria, the unconditional coverage $\left(L R_{u c}\right)$, the independency of the violations $\left(L R_{\text {ind }}\right)$ and the conditional coverage criteria $\left(L R_{c c}\right)$. To perform these tests a violations sequence is defined as follows:

$$
I_{t}(q)=\left\{\begin{array}{l}
1, \text { if } r_{t}<-\operatorname{VaR} R_{t}(q) \\
0, \text { if } r_{t}>-\operatorname{VaR} R_{t}(q)
\end{array}\right\}
$$

The unconditional coverage criterion tests whether the empirical violations are consistent with the expected ones. Thus this criterion tests the null hypothesis $H_{0, u c}: E\left[I_{t}(q)\right]=q$ against the $H_{1, u c}: E\left[I_{t}(q)\right] \neq q$. This hypothesis test is based on the following likelihood ratio test:

$$
L R_{u c}=-2 \ln \left(\frac{(1-q)^{T_{0}} q^{T_{1}}}{\left(1-\frac{T_{1}}{T}\right)^{T_{0}}\left(\frac{T_{1}}{T}\right)^{T 1}}\right) \sim \chi_{1}^{2}
$$

where $T$ is the number of out-of-sample observations, $T_{0}$ the number of non violations and $T_{1}$ the number of violations. The independence criterion tests the interrelationship between the empirical violations. This test is performed using the following likelihood ratio:

$$
L R_{\text {ind }}=-2 \ln \left(\left(1-\frac{T_{1}}{T}\right)^{T_{0}}\left(\frac{T_{1}}{T}\right)^{T 1}\right)+2 \ln \left(\left(1-\pi_{01}\right)^{T_{00}} \pi_{01}^{T_{01}}\left(1-\pi_{11}\right)^{T_{10}} \pi_{11}^{T_{11}} \sim \chi_{1}^{2}\right.
$$

where $T_{i j}$ with $i, j=0$ (noviolation), 1 (violation) is the number of observed events with the $j$ event following the $i$ event. The estimates of the probabilities of $T_{i j}$ are marked as $\pi_{01}$ and $\pi_{11}$. The conditional coverage criterion is essentially a synthetic criterion which tests the unconditional coverage and independence simultaneously. This test is performed using the following likelihood ratio:

$$
L R_{c c}=L R_{u c}+L R_{i n d} \sim \chi_{2}^{2}
$$

Another approach of evaluating the statistical performance of the VaR methods is the Engle and Manganelli (2004) Dynamic Quantile (DQ) approach. This method is based on a quantile regression model, by which the observed violations are associated with past violations and past information according to the following procedure:

$$
\begin{gathered}
\operatorname{Hit}_{t}(q)=I_{t}(q)-q \\
\operatorname{Hit}_{t}(q)=\left\{\begin{array}{l}
1-q, i f r_{t}<-\operatorname{VaR} R_{t}(q) \\
-q, i f r_{t}>-\operatorname{VaR}(q)
\end{array}\right\} \\
\operatorname{Hit}_{t}(q)=\delta+\sum_{j=1}^{K} \beta_{j} H i t_{t-j}(q)+\sum_{j=1}^{K} \gamma_{j} \zeta_{t-j}+\varepsilon_{t}
\end{gathered}
$$

where $\operatorname{Hit}_{t}(q)$ denotes the modified violations sequence, $\delta$ is a constant term and $\zeta_{t-j}$ the variable that corresponds to any information that can be derived from the existing sample 
of observations. The null hypothesis of independence, $D Q_{i n d}$, dictates that $\beta_{j}=\gamma_{j}=0$, $\forall j=1 \ldots K$., while the null hypothesis, $D Q_{u c}$, for the number of violations dictates that $\delta=0$. Therefore, the null hypothesis of the conditional coverage criterion $D Q_{c c}$ is defined as follows:

$$
D Q_{c c}=\left(\frac{\Psi^{\prime} Z^{\prime} Z \Psi}{q(1-q)}\right) \sim \chi_{2 K+1}^{2}
$$

where $\Psi$ is the $2 K+1$ parameter vector and $Z$ denotes the matrix of the regression variables. In this paper we set the maximum number of $K$ lags equal to 3 .

Another approach of evaluating the statistical performance of the VaR methods is the Christoffersen and Pelletier (2004) duration approach. While the Christoffersen and EngleManganelli approaches view the violation as the event, the duration approach takes into account the time interval between two violations. In other words, it evaluates the independence and conditional coverage hypothesis by testing the statistical properties of the sequence of time intervals between violations. The duration approach is based on the rationale of dependence causing violations clusters. In order to perform the tests, we define $d_{v}$ as the time interval between the $v-1$ and the $v$ violation. In the conditional coverage case, the evaluated method should produce exactly $q$ violations equally time distanced. Consequently the violation sequence will be characterized by a distribution with no memory. This entails that the $d_{v}$ sequence will follow the binomial distribution, i.e.

$$
f\left(d_{v}, q\right)=q(1-q)^{d_{v}-1}, d_{v} \in N
$$

It is obvious that this evaluation method is based on determining a no memory distribution for the time intervals between the violations. In other words the probability of a violation at time $t$ does not depend on the number of days that have passed since the previous violation. The only continuous distribution which is characterized by lack of memory is the exponential distribution given by the following formula

$$
f\left(d_{v}, q\right)=q e^{-q d_{v}}
$$

Christoffersen and Pelletier (2004) considered the Weibull distribution with parameters $a, b$ for the alternative hypothesis of the non conditional coverage; ${ }^{6}$

$$
w\left(d_{v}, a, b\right)=a^{b} b d_{v}^{b-1} e^{-a d_{v}^{b}}
$$

In other words, the duration approach tests of independence and conditional coverage are converted in a Weibull parameter estimation procedure. Consequently the null hypothesis of the independence $\left(D u r_{i n d}\right)$ is not rejected if $b=1$. In addition the conditional coverage $\left(D u r_{c c}\right)$ hypothesis is not rejected if $b=1$ and $a=q$.

So far the unconditional coverage tests outlined have taken into account only the number of violations, while disregarding the severity of the violations, which might prove significant in

\footnotetext{
${ }^{6}$ The Exponential distribution can be derived from the Weibull distribution for $b=1$.
} 
the case of extreme losses. To address this shortcoming, Colletaz et al. (2013) proposed a test which takes into account the severity of each violation. Specifically a second violation sequence is defined as follows

$$
J_{t}=\left\{\begin{array}{l}
1, \text { if } r_{t}<-V a R_{t}\left(q^{\prime}\right) \\
0, \text { if } r_{t}>-\operatorname{VaR}\left(R_{t}\right)
\end{array}\right\}, q^{\prime}<q
$$

where $q^{\prime}$ is a stricter significance level. The second violations or super exemptions sequence aims at measuring the number of initial violations that exceed the second threshold $V a R_{t}(q)$. Thus, if the VaR method produces an acceptable number of violations in conjunction with an increased number of super exemptions the null hypothesis of the test will be rejected. To perform the test, three indicator functions are introduced :

$$
\begin{gathered}
g_{0, t}=1-g_{1, t}-g_{2, t}=1-I_{t} \\
g_{1, t}=I_{t}-J_{t}=\left\{\begin{array}{c}
1, i f-V a R_{t}\left(q^{\prime}\right)<r_{t}<-\operatorname{VaR} R_{t}(q) \\
0, \text { if } r_{t}<-\operatorname{Va} R_{t}\left(q^{\prime}\right)
\end{array}\right\} \\
g_{2, t}=J_{t}=\left\{\begin{array}{c}
1, \text { if } r_{t}<-\operatorname{VaR} R_{t}\left(q^{\prime}\right) \\
0, \text { if } r_{t}>-\operatorname{VaR} R_{t}\left(q^{\prime}\right)
\end{array}\right\}
\end{gathered}
$$

The above random variables follow the Bernoulli distribution with $1-q, q-q^{\prime}, q^{\prime}$ parameters respectively. The null hypothesis of the test is defined as $H_{0, m u c}: E\left[I_{t}(q)\right]=q$ and $E\left[J_{t}\left(q^{\prime}\right)\right]=q^{\prime}$ and the test is performed via the following likelihood ratio:

$$
L R_{\text {muc }}=-2 \ln \left((1-q)^{N_{0}}\left(q-q^{\prime}\right)^{N_{1}}\left(q^{\prime}\right)^{N_{2}}\right)+2 \ln \left(\left(1-\frac{N_{0}}{T}\right)^{N_{0}}\left(\frac{N_{1}}{T}\right)^{N_{1}}\left(\frac{N_{2}}{T}\right)^{N_{2}}\right) \sim \chi_{2}^{2}
$$

where $N_{i, t}=\sum_{t=1}^{T} g_{i, t}, i=0,1,2$.

\subsection{Performance Evaluation}

The statistical evaluation tests sort the VaR methods according to their ability to produce the correct number of uncorrelated violations. However, they cannot provide information about the performance of the methods both in terms of underestimation or overestimation of the required capital. Another issue that arises is which method is the appropriate one in the case of multiple methods meeting the statistical criteria. To address these issues numerous loss functions and tests have been proposed. ${ }^{7}$ In this paper we adopt the newly proposed approach of Sener et al. (2012) in order to rank the VaR methods. The main advantage of this approach is that it allows for a weighting between the underestimation and overestimation error. In order to rank the methods, penalization functions grade the underestimation error $\left(r_{t}-(-V a R)<0\right)$ and the overestimation error $\left(r_{t}-(-V a R)>0\right)$. These quantities represent, for a long position, the unexpected loss and the excessive allocated capital, respectively.

\footnotetext{
${ }^{7}$ See for example, Angelidis and Benos (2008) who employ standard forecast evaluation methods in order to examine whether any differences between competing models are statistically significant.
} 
For the violation space, i.e. for all the violations, clusters of successive violations are constructed in order to compute the severity of the unexpected loss associated with each string of violations. For non-successive violations, the clusters are single elements. Let us assume that the $i-t h$ cluster has $z_{i}$ successive violations. The severity of unexpected losses for the $i-t h$ cluster of violations is calculated as follows:

$$
C_{i}=\prod_{b=1}^{z_{i}}\left(1+\left(V a R_{b, i}-r_{b, i}\right)\right)-1, i=1,2, \ldots, \alpha
$$

where $V a R_{b, i}-r_{b, i}$ denotes the error corresponding to each violation $\left(\right.$ for $\left.b=1,2, \ldots, z_{i}\right)$ of the $i$-thcluster and $\alpha$ is the number of clusters. In addition to the severity of each cluster the proposed methodology takes into consideration the correlation between clusters. So the penalization function for the unexpected loss is calculated as

$$
\begin{aligned}
\Phi\left(V a R, r_{t}\right) & =\sum_{i=1}^{\alpha-1} \sum_{m=1}^{\alpha-i} C_{i} C_{i+m} \\
& =\sum_{i=1}^{\alpha-1} \sum_{m=1}^{\alpha-i} \frac{1}{d_{i, i+m}}\left(\prod_{b=1}^{z_{i}}\left(1+\left(V a R_{b, i}-r_{b, i}\right)\right) \prod_{b=1}^{z_{i+m}}\left(1+\left(V a R_{b, i+m}-r_{b, i+m}\right)\right)-1\right)
\end{aligned}
$$

where $d_{i, i+m}$ is the distance in days between the clusters. The penalization function of overestimation error is derived in a similar way. However for this type of error the correlation between the excessive allocations does not affect directly the position, so it is not important to the computation of the excessive allocated capital penalization function. The penalization function for the overestimation of VaR is calculated as

$$
\Psi\left(V a R, r_{t}\right)=\sum_{t=1}^{T}\left[I\left(r_{t}>-V a R \mid r_{t}<0\right)\right]\left(V a R-r_{t}\right)
$$

The penalization measure is calculated as

$$
P M\left(\Theta, r_{t}, V a R\right)=\frac{1}{T^{*}}\left[(1-\Theta) \Phi\left(V a R, r_{t}\right)+\Theta \Psi\left(V a R, r_{t}\right)\right]
$$

where $\Theta$ denotes the weight on each penalization function and $T^{*}$ denotes the number on non positive returns. For this paper $\Theta$ has been set equal to the VaR estimates significance level $(q)$. The $P M$ ratio for the $j$ th method is defined as

$$
P M_{j-\text { ratio }}=\frac{P M_{j}}{\sum_{j=1}^{n} P M_{j}}
$$

The method with the lowest ratio is the best performing one. Thus the ranking is a descending sort of the ratios. In addition to the ranking methodology, Sener et al. (2012) propose a supplementary test in order to test statistically the equality of performances between candidate methods. The main advantage of this test is the use of a ratio which eliminates the use of a benchmark method and reduces the computation complexity. For the supplementary test, the 
PM ratio is set as the loss function and the loss series for the $j$-th method is defined as

$$
\left\{k_{j, t}\right\}_{t=1}^{T}=\left\{\frac{P M_{j, t}}{\sum_{j=1}^{n} P M_{j, t}}\right\}_{t=1}^{T}
$$

If all the methods perform equally well, then the above ratio should be equal to $1 / n$. Hence the null hypothesis for the $j$-th method is defined as

$$
H_{0}: E\left[k_{j, t}\right] \leq \frac{1}{n}, j=1, \ldots, n
$$

and the test statistic is defined as:

$$
\begin{gathered}
W_{j}=\sum_{t=1}^{T} I\left(k_{j, t}>\frac{1}{n}\right) \\
\widehat{W}_{j}=\frac{W_{j}-p T}{\sqrt{p(1-p)}} \sim N(0,1)
\end{gathered}
$$

where $p$ is the probability of $k_{j, t}>\frac{1}{n}$ and is set at 0.5 . The above procedure reduces significantly the computational complexity. However if a number of methods produce relative high ratios, a false non rejection may occur. To remedy this disadvantage, we evaluate the methods' performance in two steps. First we calculate the PM ratios and the corresponding tests for all the implemented methods. Second, we exclude the methods with the highest PM ratio and perform the evaluation process again. Finally it has to be noted that the Ratio ranking and the supplementary test may point to different directions.

\section{Empirical Findings}

\subsection{Dataset and Descriptive statistics}

The bulk shipping sector consists the major form of sea transportation. This sector is particular segmented with the most notable categories being the dry bulk sector, which is responsible for raw non liquid materials transportation and the tanker sector which is for liquids. Each freight depends on the type of the contract, ${ }^{8}$ the size of the vessel, the type of the cargo and the route followed. This heterogeneity makes freight rate dynamics more complex than those of traditional asset classes (stocks, stock indices, etc.). A major provider of these rates is the Baltic Exchange, which publishes daily a plethora of spot freight rates calculated by members (panelists) of the exchange.

The dataset used in this study consists of eleven indices of the Baltic Exchange, describing both the aggregate state of the market and individual routes of the dry and wet cargo sectors. The aggregate indices we employ are the Baltic Dry Index (BDI), the Baltic Panamax Index

\footnotetext{
${ }^{8}$ The type of contract entails specific characteristics for the chartering of a vessel. There are four types, voyage, bareboat, time charter and contract of affreightment (COA). The voyage type consists of the simplest contract where the vessel is chartered for a specific voyage and the rate is calculated by the weight of the goods. Bareboat type contracts lease only the equipment while Time Charter contracts lease the equipment and the corresponding services. Finally, COA consists of a series of voyage contracts.
} 
(BPI), the Baltic Capesize Index (BCI), the Baltic Dirty Tanker Index (BDTI) and the Baltic Clean Tanker Index (BCTI). BDI is the general index of the dry bulk freight market and is expressed in index points. It is calculated as the non equal weighted sum of BCI, BPI and BHMI (Baltic Handymax Index) time charter averages. ${ }^{9}$ These sub-indices are composite drycargo indices across international routes that correspond to vessels of size 30-49,999, 50-79,999 and over 80,000 deadweight tones (dwt henceforth) for the BHMI, BPI and BCI, respectively. With respect to the tanker shipping sector, we employ the Baltic Dirty Tanker Index (BDTI) and the Baltic Clean Tanker Index (BCTI), which are the two major subsector indices of this market. The distinction between 'clean' and 'dirty' comes from the type of cargo and specifically from whether the oil product carried can be classified as clean or dirty. ${ }^{10}$

The aforementioned indices are aggregate indices and as such we expect their behavior to be smoother compared to their constituents similarly to the diversification effect present in a portfolio of assets. However as they are averages of individual route or time charter contract indices, they do capture the general dynamics of the segmented freight market and can be thought of as portfolios of freight rate positions covering large fleets of vessels. On the other hand, individual route indices are more relevant for market risk exposures of smaller companies, since these companies employ vessels in one of these routes which also serve as the underlying assets of freight rate derivatives. To this end, we employ two popular averages of the time chartered indices included in the calculation of BPI and BCI; namely the 4 Time Charter Average Capesize (4TC AVG CAPE) and the 4 Time Charter Average Panamax (4TC AVG PANAM). They are averages of specific routes, expressed in US dollars and measure the cost to hire the vessel per day. ${ }^{11}$ We complement our dataset with four popular Worldscale routes of the Baltic Dirty Index; ${ }^{12}$ namely route TD3 (Middle East Gulf to Japan, for Very Large Crude Carriers (VLCC) vessel sizes of 250,000 dwt), the TD5 route (West Africa to US Atlantic Coast (USAC), for Suezmax vessel sizes of 130,000 dwt), route TD7 (North Sea to Continent, for Aframax vessel sizes of 80,000 dwt) and route TD9 (Caribbean to US Gulf, for Panamax vessel sizes of 70,000 dwt). The choice of these routes and time charter averages stems from their liquidity in the freight forward and option markets. In what follows we concentrate on the behavior and analysis of the aggregate sector indices. The analysis and findings for the individual routes are presented and discussed in Section 5.

Our dataset covers the period from 1/11/1999 to 13/03/2012 including 3091 daily observations. The dataset is obtained from Clarkson's Shipping Intelligence Network. Table 1 presents the descriptive statistics of the daily logarithmic returns of the indices at hand. All series display means statistically equal to zero as it is typical with daily returns of financial assets.

\footnotetext{
${ }^{9}$ The Baltic Handymax index was replaced by the Supramax index in 2006.

${ }^{10}$ Clean products consist of lighter (sweet) distillates, such as gasoline and kerosene, which are usually shipped via vessels with coated tanks to ensure the cleanliness of the product. Dirty products involve lower distillates and residual oil which is usually shipped in conventional tankers.

${ }^{11}$ Admittedly, these indices do not respresent individual routes; rather they represent averages of individual ones. However, we loosely refer to them as 'individual' routes since they are more disaggregate indices.

For a description of the specific time chartered routes, see Table 1 in Angelidis and Skiadopoulos(2008).

${ }^{12}$ Worldscale is a freight rate measurement. It is used in the tanker sector and is calculated every year by the World Scale Association. Each rate is quoted as a precentage of 100 Worldscale.
} 
Moreover, all return series are highly volatile and leptokurtic. The tanker sector displays higher kurtosis and is more negatively skewed compared to the bulk dry sector. These features indicate an increased probability for extreme events and given the negative asymmetry in their empirical distribution (with the exception of BCI) the odds are in favour of negative outcomes. As expected, the null hypothesis of normality is rejected as indicated by the Jarque-Bera test. In addition, contrary to more traditional asset classes (stocks, stock indices, etc.) freight rate returns display significant autocorrelation as indicated by the Ljung Box statistic. Finally, the return series exhibit significant heteroscedasticity as suggested by the Ljung Box statistic for the squared return series. These characteristics of the freight return series call for a VaR methodology that can adequately capture tail risk.

\section{[TABLE 1 AROUND HERE]}

\subsection{Statistical Evaluation}

Before presenting our findings, it is useful to briefly describe our forecast construction methodology. Given a total sample of $K$ observations, we must determine the way to split the sample into the estimation part (say $R$ observations) and the out-of-sample part (say $T:=K-R$ observations). Obviously, there is a trade-off, since a large $R$ improves the quality of the estimated parameters of the model but, at the same time, leaves few observations for the out-of-sample forecast exercise making the evaluation of the predictive ability of the model difficult. In our analysis, we keep about $1 / 3$ of the available sample (1029 observations) for out-of-sample forecasting, which gives us a sufficient number of forecasts to evaluate the estimated models, while keeping enough observations to obtain reliable in sample parameter estimates. We employ a rolling forecasting scheme, i.e. the size of the estimation sample remains fixed (equal to $R=2061$ observations) and produce forecasts for $5 \%$ and $1 \%$ VaR, which are used to conduct both the statistical and the performance evaluation.

Tables 2 to 6 present the backtesting results by providing the p-values associated with the statistical evaluation of our methods (see Section 3.1). More in detail, for the BDI index and the $5 \%$ estimates (Table 2, Panel A) our results suggest that the hybrid and parametric GARCHtype methods (AR-GARCH(1,1)-Normal, AR-GARCH(1,1)-Student, AR-EGARCH(1,1)-Normal, AR-EGARCH(1,1)-Student) pass all the conditional coverage criteria while the Historical SimulationAll and Variance Covariance methods fail on the basis of all tests. Moreover, the Combination Median method succeeds in meeting all the backtesting criteria, while the remaining combination methods fail to respond adequately to the violation clustering. The failure of the mean and trimmed mean combination methods are in a sense expected, since almost half of the individual methods exhibit non tolerance to violation clustering.

\section{[TABLE 2 AROUND HERE]}

Our findings with respect to BPI (Table 3, Panel A) point to the superiority of the AR$\mathrm{ARCH}(1)-$ Normal model which succeeds in all the backtesting criteria. However, most of the parametric GARCH and hybrid methods meet the conditional coverage criteria. Despite the 
conditional coverage success, the rather low p-values of some parametric and hybrid methods in the LR and DQ independence tests suggest that the market returns display more volatile clustering. As for the combination methods we find similarities with the individual methods' results. In more detail, only the Combination Median produces statistically accurate forecasts while the remaining combination methods succeed in specific unconditional coverage tests.

\section{[TABLE 3 AROUND HERE]}

Turning to the BCI Index (Table 4 Panel A), our findings lead to similar results pointing to the superiority of parametric (GARCH type) and hybrid methods. In addition to GARCH volatility modelling, the Combination Median method meets all the criteria while the trimmed mean methods are successful in at least two conditional coverage criteria (Standard approach and Duration approach). The borderline p-values associated with the Combination Mean method and the $\mathrm{LR}_{c c}$ and $\mathrm{DQ}_{c c}$ tests are due to some extreme values produced by the individual methods. This is further supported by the success on the basis of Dur ${ }_{c c}$ and most importantly by the success of the trimmed methods which by construction exclude extreme forecasts.

\section{[TABLE 4 AROUND HERE]}

Moving to the $1 \%$ significance level, we find that the majority of individual methods are statistically rejected while the combination methods display superior performance. For the BDI index (Table 2, Panel B) we find that only the AR-GARCH(1,1)-Student and AR-EGARCH(1,1)Student models pass all the backtesting criteria while the Filtered Historical Simulation method fails only on the basis of the $\mathrm{LR}_{\text {muc }}$ test. For the combination methods we find that the Combination Mean and Combination Trim1 methods pass all the criteria while the Combination Median and Combination Trim2 methods fail the DQ2 $2_{c c}$ and DQ3 $3_{c c}$ tests, respectively. As far as the BPI index is concerned (Table 3, Panel B), mainly the parametric and hybrid methods produce statistically accurate forecasts with the AR-GARCH(1,1)-Student, AR-EGARCH(1,1)Student and hybrid methods passing all the criteria. The Combination Median method emerges as the best among the combination methods followed by the Combination Mean and Combination Trim2. Our findings regarding the BCI index (Table 4, Panel B) are similar to the BDI index, where the AR-GARCH(1,1)-Student, AR-EGARCH(1,1)-Student and hybrid methods pass all the backtesting criteria. More importantly, all the combination methods succeed in all the backtesting criteria.

Our findings for the tanker sector paint a different picture. Specifically, with respect to the BDTI index and the 5\% VaR level, (Table 5, Panel A) only the Filtered Historical Simulation method passes all the conditional coverage criteria. All the remaining individual methods, with the exception of EVT, exhibit fewer violations which eventually lead to rejection of the Conditional Coverage Hypothesis. Similarly, the combination methods overestimate VaR and lead to a significantly reduced number of violations. However the combination methods produce very good results in terms of independence, indicating their suitability to correctly capture 
violation clustering. Similar findings pertain to the case of BCTI with the addition of three more statistically accepted individual methods. More in detail, for the Clean tanker subsector (Table 6, Panel A) the Filtered Historical Simulation method passes all the backtesting criteria while the Monte Carlo Simulation passes all the conditional coverage criteria. Finally the AR-GARCH(1,1)-Student and the AR-EGARCH(1,1)-Student models pass two conditional coverage criteria. Despite the improved statistical performance of some individual methods, the performance of the combination methods does not show any signs of improvement. As in the BDTI case, the overestimation of market risk leads to a decreased number of violations.

\section{[TABLE 5 AROUND HERE]}

Moving to the $1 \%$ significance level we have to note that our findings are quite different compared to the 5\% significance level. For BDTI (Table 5, Panel B), eleven of the individual methods pass at least two conditional coverage criteria, while six pass all the backtesting criteria. In addition, Variance Covariance, Historical SimulationAll and Historical Simulation-500 pass all the LR criteria and individual DQ tests. More importantly, the combination methods display an improved performance. Specifically, the CombinationTrim2, Combination Mean and Combination Median methods fail only on the basis of the $\mathrm{LR}_{m u c}$ test. With respect to the Combination Trim1 method we should clarify that its failure at the $\mathrm{LR}_{u c}$ and $\mathrm{DUR}_{u c}$ test is attributed to the overestimation of risk. In addition, the successes at all the DQ $\mathrm{Q}_{c c}$ and independence tests, the borderline failure at the $\mathrm{LR}_{c c}$ and finally the second highest $\mathrm{LR}_{m u c} \mathrm{p}$-value suggest that Combination Trim1 can be considered as a statistically adequate method. The results for the BCTI Index (Table 6, Panel B) are quite similar but with fewer successes in the individual methods. All the parametric and hybrid methods pass at least two conditional coverage criteria while the Historical Simulation-250, Historical Simulation-500 and the Variance Covariance methods fail. With respect to the combination methods, we find an improved performance compared to BDTI since they all pass at least two conditional coverage criteria and three of them pass all the conditional coverage criteria.

\section{[TABLE 6 AROUND HERE]}

\subsection{Performance Evaluation}

While the statistical evaluation framework provides a way of examining the statistical accuracy of the VaR methods, it does not offer any insight on the economic performance of the alternative methods with the exception of the Colletaz et al. (2013) test. In other words, the statistical backtesting framework does not offer a measure of overestimation or underestimation of market risk, which in turn is crucial in selecting the most reliable/suitable method. In this paper, we employ the performance evaluation approach proposed by Sener et al. (2012), described in detail in Section 3.2. The evaluation proceeds in two steps. First, we derive the loss ratio and the supplementary test for all the methods. Our findings are reported in Table 7 (Panels A and B for the $5 \%$ and $1 \%$ VaR, respectively). At a second step, we repeat the analysis by excluding 
the worst performing methods, i.e. those with significantly high ratios. In this way, we make sure that we get more accurate results which are not affected by extreme ratios.

\section{[TABLE 7 AROUND HERE]}

For the BDI index and the 5\% VaR level, the Penalization Measure (PM) (Equation 34) ranges from 0.135 for the AR-EGARCH-N model to 0.592 for the HistoricalAll simulation method. The corresponding ratios (Equation 35) range from 3.255 to 14.265. Overall, our findings suggest that the non-parametric Variance Covariance methods and the AR-ARCH-t methods are outperformed by the parametric, hybrid and combination methods. With respect to the combination methods we have to note that they belong to the pool of the equally performing methods with the Combination median performing best. Excluding the methods with the relative worst PM ratios (Table 8, Panel A), we find that the AR-ARCH methods, the Riskmetrics and the EVT are outperformed by the remaining methods while the initial ranking is maintained. At the 1\% VaR level (Table 7, Panel B) our findings are similar with the Variance Covariance, the AR-ARCH-N and most of the non-parametric methods being outperformed by the remaining methods. However, at the $1 \%$ significance level the ranking of the accepted methods differ in comparison with the $5 \%$ level. Specifically the three highest performing methods are the mean combination methods while the median ranks fifth. Excluding the worst ranked methods (Table 8 Panel B), we find that the Riskmetrics, the Historical Simulation 500 and three of the parametric methods are statistically out performed while the equal performing methods maintain their ranking.

\section{[TABLE 8 AROUND HERE]}

For the BPI index, our 5\% VaR level results are generally similar to the BDI index. The majority of the parametric and hybrid methods outperform the non-parametric ones, the ARARCH-t and Variance Covariance methods, while the combination methods can not be rejected with the median method ranking third. Excluding the worst performing methods, we find that the GARCH-type methods, the Filtered Historical Simulation, the Monte Carlo Simulation, the Median and Trim2 Combination methods emerge as superior methods. Moving to the $1 \%$ VaR level (Table 7, Panel B) we find that the Historical SimulationAll, the Historical Simulation500 and the Variance Covariance methods are the worst performing closely followed by the Historical Simulation-250, the Riskmetrics, the AR-ARCH(1)-N and the mean Combination method. Excluding the worst performing methods (Table 8, Panel B) we find that the pool of rejected methods is joined by the one year Historical Simulation, Riskmetrics, AR-ARCH(1)-t, Mean and Trim2 combination methods.

Turning to the BCI index (Table 7, Panel A), our findings suggest that at the $5 \%$ significance level two of the nonparametric methods, the Variance Covariance plus the AR-ARCH methods are outperformed by the remaining ones. In addition we find that the Combination Median method ranks first and the alternative combination methods rank from the fourth to the sixth place. The re-evaluation of the methods (Table 8, Panel A) suggests that no specific group of 
methods is superior while the rejected methods consist of the non-parametric two year Historical Simulation, the Riskmetrics, the parametric EVT and the AR-ARCH-N methods. At the $1 \%$ VaR level (Table 7, Panel B) our findings are similar. The combination methods produce mixed signals since the Combination Trim1 method ranks first while the remaining combination methods rank from the seventh to the ninth position. The second step evaluation (Table 8, Panel B) does not yield any differences in the rankings of the methods with the combination methods being statistically equal to the best performing methods.

For the BDTI index at the 5\% significance level, we find that the non-parametric, the Variance Covariance, the EVT and AR-EGARCH-N are outperformed by the remaining methods, while the combination methods rank within the first five places with the Combination Mean method ranking first. Our second step evaluation findings suggest that the combination methods perform better than any other group of methods. Four of the parametric methods and one of the hybrid ones are outperformed by the remaining methods which in general attain their first step evaluation ranking. Our $1 \%$ significance level results show that the rejected methods are the same as previously with the exception of the EVT and Historical Simulation One Year. The second step evaluation (Table 8 Panel A) does not yield any substantial differences. However the combination methods do not perform as well as at the $5 \%$ significance level despite the third place of the Trim1 method and the fact that only the median method is rejected.

For the BCTI index and the 5\% VaR level (Table 7, Panel A) six of the individual methods are rejected including the non-parametric methods, the Variance Covariance and representatives from the other groups of methods with the exception of the combination methods. Specifically, the combination methods occupy the four top positions with the Combination Median method ranking first. Excluding the worst performing methods (Table 8 Panel A) we find that the combination methods and the parametric ones with normal realizations outperform the remaining methods. With respect to the $1 \%$ significance level (Table 7, Panel B) we show that except for the non-parametric methods and the Variance Covariance methods, the remaining methods perform equally well. Moreover, the first two positions are occupied by the Trimmed Combination methods while the Combination Median and Combination Mean rank sixth and seventh respectively. At the second step evaluation (Table 8, Panel B) we find that six methods are rejected including the parametric ones with normal errors, the EVT, the Riskmetrics and finally the Filtered Historical Simulation.

\subsection{Joint Evaluation}

The findings presented so far are indicative of the statistical and performance evaluation independently. However, in order to evaluate our methods in a comprehensive way and judge their overall ability to measure market risk accurately and efficiently, we have to combine the results of the statistical and performance evaluation. Towards this direction we rank the statistically accepted methods, presented in Section 4.2 by employing the performance evaluation ranking results presented in Section 4.3. As already mentioned, we consider as a statistically accepted method any method that has passed at least two conditional coverage criteria. Table 9 presents 
the ranking of the statistically accepted methods for the two significance levels.

For the 5\% significance level (Table 9, Panel A) we could conjecture that due to the timevarying nature of volatility, the methods with a GARCH-EGARCH-Normal volatility mapping cope better and produce superior results in contrast with the non-parametric ones. Concerning the combination methods, the combination Median performs equally well in every case while it ranks first in the BCI case. Moving to the Tanker sector our findings differ mainly due to the nature of the returns of the corresponding indices. Because of the overestimation of risk, the final evaluation of the methods for the BDTI and BCTI indices include the methods that overestimate market risk. The respective findings show that combination methods outperform the individual ones. Overall the results in the dry and tanker sector suggest that the Combination Median can be considered as a global method of measuring market risk or at least as an accurate proxy for the expected daily $5 \% \mathrm{VaR}$ of the freight rates.

\section{[TABLE 9 AROUND HERE]}

Our findings with respect to the $1 \%$ significance level (Table 9, Panel B) paint a different picture. For both sectors the combination methods rank amongst the top positions ${ }^{13}$ alongside specific individual methods. Contrary to the $5 \%$ significance level, the parametric methods with GARCH-EGARCH-Student volatility mapping perform better than the non-parametric, other parametric methods and the hybrid methods. However the very good performance of these individual methods cannot mask the superior performance of the combination methods and especially the Combination Trim1 method, which ranks first in three indices and third in one. At this point, we should mention that the failure in BPI is due to overestimation of risk which in turn leads to the failure of the unconditional coverage hypothesis. However the independence criteria point to the adequacy of the method, a fact that is supported by both the PM ratio and the performance equality test. ${ }^{14}$

To conclude, we find that the combination methods are a strong alternative to the plethora of individual methods. At the $5 \% \mathrm{VaR}$ we find that the combination methodology provides a globally accepted method, namely the Combination Median method, which in every index under scrutiny performs equally well to the highly-ranked individual methods and thus can significantly reduce the cost of freight rate risk measurement. For the $1 \% \mathrm{VaR}$, we find that the combination methods display superiority over the individual methods with Combination Trim1 attaining the highest performing ranking in almost every case.

\footnotetext{
${ }^{13}$ The acceptance of the Combination Trim 1 method as an accurate method is due to the borderline backtesting results (see section 4.2) in conjunction with the superior results in the evaluation ranking.

${ }^{14}$ In contrast with the Combination Trim1 in the BDTI case, for the BPI the combined results of the statistical and performance evaluation are not sufficient to surpass the "at least two Conditional Coverage criteria pass" rule and therefore consider the method as an adequate one.
} 


\section{Robustness Checks: Individual routes}

In order to examine the robustness of our main findings we implement our analysis for the six individual routes already described in section 4.1. ${ }^{15}$ Quite importantly, individual routes are practically relevant from the shipping industry perspective since ships can be fixed on a floating rate charter based on the daily value of the Baltic time charter averages, for example.

Starting with the time chartered averages, we note that their heteroskedastic and heavy tailed profile leads to failure of the majority of the individual methods. For the 4 TC AVG CAPE, only the AR-GARCH, AR-EGARCH and Monte Carlo Simulations methods prove adequate. Despite the failure of the majority of the individual methods, the Combination Median method produces excellent results succeeding in every backtesting criteria. Similar results are reported for the 4 TC AVG PANAM 5\% statistical evaluation where the AR-ARCH-Normal, AR-GARCH, Filtered Historical and Monte Carlo Simulation methods pass all the backtesting criteria. However in this case none of the combination methods produce statistically accurate results. Turning to the individual tanker routes, our findings suggest that the majority of the parametric and hybrid methods are statistically accurate for the TD5, TD7 and TD9 routes. Quite importantly, the proposed combination methods produce excellent results. In contrast with the aforementioned three routes, only the student-t parametrized methods prove adequate for TD3, as the remaining methods overestimate risk.

Moving to the $1 \%$ statistical evaluation, the 4 TC AVG CAPE results suggest that there is a significant improvement in the statistical performance of the combination methods. Combination Mean, Combination Trim 1 and Combination Trim 2 produce statistically accurate results, while only the AR-GARCH-Student and the Montecarlo Simulation are qualified as statistically adequate individual methods. For the 4 TC AVG PANAM, the AR-GARCH-EGARCH and Hybrid methods, produce accurate results. In addition three of the combination methods, namely Combination Mean, Combination Median and Combination Trim 2, produce accurate results with Combination Median passing all the backtesting Criteria. A similar improvement is also evident for the individual tanker routes, where the majority of the individual methods and all the combination methods produce accurate results, even for the TD3 case.

The first step of the performance evaluation for both sectors produces results similar to the statistical evaluation ones. Specifically, at the 5\% significance level, the 4 TC AVG CAPE results suggest that the parametric (AR-GARCH, AR-EGARCH), hybrid and combination methods perform equally well to the best performing method. Similarly, the 4 TC AVG PANAM results are almost identical with the addition of EVT, Riskmetrics and AR-ARCH-Normal. For the Tanker Sector the student parameterization seems to underperform while in some cases (TD3, TD5) the non-parametric Historical Simulation 250 and Historical Simulation 500 perform well. In addition, the Riskmetrics is performing equally in every index while the Variance Covariance is not rejected only for the TD5 index. At the $1 \%$ significance level, our results are similar with the combination methodology producing non-rejection results in every case under scrutiny. The

\footnotetext{
${ }^{15}$ To save space, we do not include detailed tables in this paper. These tables are available from the authors upon request.
} 
hybrid and parametric GARCH-EGARCH-student methods produce a single rejection in the TD3 index while the Variance Covariance is rejected in every case. Finally it is worth mentioning that there is at least one non parametric method performing equally well in every index.

The second step evaluation results are consistent with the first step results. At the 5\% significance level the Dry Bulk results are almost identical for the parametric AR-GARCH, AR-EGARCH and Hybrid methods. However in the Panamax Sector there is a rejection of performance equality for the Combination Mean, Combination Trim 1 and Combination Trim 2 methods. At the tanker sector there are rejections of equal performance for the parametric methods with student-t parametrization, while the combination methodology performs equally in almost every case. An exception has to be made for the TD5 index where the Combinations Trim methods fail to perform equally well. At the $1 \%$ level evaluation only the Filtered Historical Simulation presents a non rejection in every case while the Montecarlo and the AR-GARCHStudent methods produce a rejection in TD3 index. The remaining individual methods do not produce a specific profile as there are random rejections and non-rejections. Finally the performance of the proposed combination methodology is encouraging with at least one nonrejection in every case and four non rejection in three routes (4 TC AVG CAPE, TD3, TD5).

Combining the results of the statistical and the performance evaluation clarifies the overall performance of the implemented methods and reveals that the combination methods pose a strong alternative especially at the $1 \%$ significance level. Specifically at the $5 \%$ significance level, there is not a single method that produces a global profile. Alongside the Combination Median, which is equally performing with the top ranked method in four indices, the ARGARCH-Normal produces similar results. In addition, our combination methods perform better in the tanker sector where for the TD5, TD7 and TD9 indices there are at least two methods in the best performers' club. Moving to the $1 \%$ significance level, our results are in favor of the proposed combination methodology. Specifically in every case under scrutiny there is a statistically accepted best performing combination method with Combination Trim1 alongside AR-GARCH-Normal presenting a global profile.

To conclude, measuring the market risk of the individual routes is quite challenging due to the specific characteristics attached to each one of these routes. Our findings imply that although there is not a definite superiority of the combination methodology, it poses a reliable alternative that reduces significantly model uncertainty and parameter instability contained in the individual methods. This is even more prominent in the $1 \%$ VaR calculation where the combination methodology provides, in every case under scrutiny, at least one statistically accepted method performing equally well to the best performing one. These results confirm the superiority of the proposed combination methodology and the robustness of our approach, a finding that reinforces its potential suitability for other markets such as the freight derivatives market and a variety of financial markets. 


\section{Conclusions}

Freight rate risk is one of the most important risk factors of the shipping industry. In addition the profile of the distributions of returns complicate the measurement of freight risk leading to inefficient performances by the majority of the VaR methods. In this paper we provided a thorough insight of freight risk via a VaR methodology. Specifically we considered the performance of the standard VaR methods amid the recent adverse economic circumstances while most importantly we proposed a Combination approach aiming at superior VaR results. In order to evaluate the employed models/ methodologies, we implemented a two step evaluation methodology. For the backtesting of the implemented methods, three distinct approaches plus a newly proposed test were applied in order to achieve an in depth statistical evaluation. Specifically besides the standard approach proposed by Christoffersen (1998) we implemented the Dynamic Quantile approach proposed by Engle and Manganelli (2004), the Duration approach proposed by Christoffersen and Pelletier (2004) and finally the super exemption test proposed by Colletaz et al. (2013).

For the evaluation of the forecasting performance, we implemented a newly proposed methodology introduced by Sener et al. (2012). The main advantages of the this methodology, in contrast with the standard loss functions, are two. First it allows weights to each type of error, making it possible to distinguish between underestimation and overestimation errors. Secondly it significantly reduces the computation complexity associated with the testing of performance equality.

Our findings suggest that on the basis of individual methods only the parametric and hybrid methods produced acceptable results and seem to adapt better to the volatile nature of the freight market. More importantly we found that the combination methods produce better results than the individual methods posing a strong alternative to the plethora of individual methods. In addition we found combination methods that present a global profile applicable throughout the entire freight market. Therefore the combination methods we propose can provide accurate results while simultaneously reducing the cost of model evaluation and selection.

Further research should investigate the possibility of expanding the model space to incorporate models based on realized volatility estimators, such as the AR-RV, HAR-RV and LHAR-RV models (Andreou et al., 2012). In this respect intra-day data should be employed, which unfortunately are currently unavailable due to the nature of the spot freight rate market. In addition, such an approach can be pursued for the evaluation of risk in futures freight contracts for which it might be probable that IMAREX provides intra day data in the near future. Finally, current revisions to the Basel III framework suggest moving from VaR to Expected Shortfall. In this respect, it would be worth investigating whether our framework can be of sufficient value in this new era for risk management practices. 


\section{References}

[1]Abouarghoub, W., Mariscal, I. B.-F., \& Howells, P. (2014). A two-state Markov-switching distinctive conditional variance application for tanker freigth returns. International Journal of Financial Engineering and Risk Management, 1, 239-263.

[2] Alizadeh, A., H. (2013). Trading volume and volatility in the shipping forward freight market. Transportation Research Part E: Logistics and Transportation Review, 49, 250-265.

[3]Alizadeh, A., H. , Huang, C.-H., \& Dellen, S. (2014) A regime switching approach for hedging tanker shipping rates. Energy Economics, 49 (10), 44-59.

[4]Alizadeh, A., H., \& Nomikos N. (2009). Shipping dericatives and risk management. Palgrave Mcmillan, New York.

[5]Alizadeh, A., H., \& Nomikos N. (2011). Dynamics of the Term Structure and Volatility of Shipping Freight Rates. Journal of Trasport Economics and Policy, 45 (1), 105-128.

[6]Andreou, E., Kourouyiannis, C., \& Kourtellos, A. (2012). Volatility forecasts combinations using asymmetric loss functions. University of Cyprus, Working Paper in Economics, WP1712.

[7]Angelidis, T., \& Benos, A. (2008). Value-at-Risk for Greek Stocks. Multinational Finance Journal, $12(1 / 2) 67-104$.

[8]Angelidis, T., \& Skiadopoulos, G. (2008). Measuring the Market Risk of Freight Rates: A Value-At-Risk Approach. International Journal of Theoretical and Applied Finance, 11:5, 447-469.

[9]Barone-Adesi, G., Giannopoulos, K., \& Vosper, L. (1999). VaR without Correlations for Nonlinear Portfolios. Journal of Futures Market, 19, 583-602.

[10]Becker, R., \& Clements, A. (2008). Are Combination Forecasts of S\&P500 Volatility Superior? International Journal of Forecasting, 24, 122-133.

[11]Bessis, J. (2002). Risk Management in Banking 2nd Edition. Wiley.

[12]Bollerslev, T. (1986). Generalized Autoregressive Conditional Heteroskedasticity. Journal of Econometrics, 31, 307-327.

[13]Christoffersen, P. (1998). Evaluating Interval Forecast. International Economic Review, 39, 841-862.

[14]Christoffersen, P., Pelletier, D. (2004). Backtesting Value-at-Risk: A Duration-Based Approach. Journal of Financial Econometrics, 2, 84-108.

[15]Colletaz, G., Hurlin, C., \& Perignon, C. (2013). The Risk Map: A New Tool for Backtesting Value-at-Risk Models. Journal of Banking and Finance, 37 (10), 3843-3854. 
[16]Corsi, F. (2009). A Simple Approximate Long-Memory Model of Realized Volatility. Journal of Financial Econometrics, 7, 174-196.

[17]Corsi, F. \& R. Reno (2009). Har volatility modelling with heterogeneous leverage and jumps, Working paper, University of Siena.

[18]Engle, R. (1982). Autoregressive Conditional Heteroskedasticity with Estimates of the Variance of U.K Inflation. Econometrica, 50, 987-1008.

[19]Engle, R. F., \& Manganelli, S. (2004). CAViaR: Conditional Autoregressive Value at Risk by Regression Quantiles. Journal of Business and Economic Statistics, 22, 367-381.

[20]Goulas, L., \& Skiadopoulos, G. (2012). Are Freight Futures Markets Efficient? Evidence from IMAREX. International Journal of Forecasting, 28, 644-659.

[21]Halbleib-Chiriac, R., \& Winfried P. (2012). Improving the Value at Risk Forecasts: Theory and Evidence from the Financial Crisis. Journal of Economic Dynamics and Control, 36 (8), $1212-1228$.

[22]Huang, H., \& Lee, T., (2010). To combine Forecasts or to combine Information?. Econometric Reviews, 29, 534-570.

[23]Kavussanos, M., \& Dimitrakopoulos, D. (2011). Market Risk Model Selection and Medium Term Risk with Limited Data: Application to Ocean Tanker Freight Markets. International Review of Financial Analysis, 20, 258-268.

[24]Kavussanos, M., \& Visvikis, I. (2004). Market interactions in returns and volatilities between spot and forward shipping freight derivatives. Journal of Banking and Finance, 28, 20152049 .

[25]Kavussanos, M. G., \& Visvikis, I. D. (2006). Shipping freight derivatives: a survey of recent evidence. Maritime Policy and Management, 33, 233-255.

[26]McAleer, M., Jimenez-Martin, J. A., \& Perez-Amaral T. (2013). International Evidence on GFC-Robust Forecasts for Risk Management under the Basel Accord. Journal of Forecasting, $32(3), 267-288$.

[27]Nomikos N., Kyriakou, I., Papapostolou, N., \& Pouliasis P. (2013). Freight Options: Price Modelling and Empirical Analysis. Transportation Research Part E: Logistics and Transportation Review, 51, 82-94.

[28]Nelson, D. (1991). Conditional Heteroskedasticity in Asset Returns: A new approach. Econometrica, 59, 347-370.

[29]Patton, A., Sheppard, K., (2009). Optimal combinations of realised volatility estimators. International Journal of Forecasting, 25, 218-238. 
[30]Rapach, D., Strauss, J., \& Zhou, G. (2010). Out of Sample Equity Premium Prediction:Combination Forecasts and Links ti the Real Economy. The Review of Financial Studies, $23: 2,821-862$.

[31]Sener, E., Baronyan, S., \& Menguturk, L. (2012). Ranking the Predictive Performances of Value at Risk estimation methods. International Journal of Forecasting, 28, 849-873.

[32]Stock, J. H., \& Watson M.W. (2004). Combination Forecasts of Output Growth in a SevenCountry Data Set. Journal of Forecasting, 23, 405-430.

[33]Stopford, M. (2009). Maritime economics 3rd ed. Routledge, Oxon.

[34]Timmermann, A. (2006). Forecast combinations. In G.Elliott, C.W. J.Granger, \& A.G. Timmermann (EBS), Handbook of economic forecasting. Amsterdam : North Holland Press. 
Table 1. Descriptive Statistics

\begin{tabular}{|c|c|c|c|c|c|}
\hline & LBCI & "LBCTI & 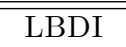 & "LBDTI & LBPI \\
\hline Mean & -0.006 & 0.000 & -0.014 & 0.004 & -0.010 \\
\hline Median & 0.000 & -0.120 & 0.057 & -0.060 & 0.048 \\
\hline Maximum & 16.502 & 25.359 & 13.658 & 22.951 & 12.836 \\
\hline Minimum & -19.215 & -29.647 & -11.953 & -38.122 & -21.623 \\
\hline Std. Dev. & 2.630 & 1.493 & 1.832 & 2.230 & 2.308 \\
\hline Skewness & 0.067 & -1.515 & -0.066 & -1.613 & -0.471 \\
\hline Kurtosis & 9.488 & 93.744 & 9.928 & 40.501 & 12.081 \\
\hline Jarque-Bera & 5423.5 & 1061713. & 6184.5 & 182467.8 & 10735.7 \\
\hline Probability & 0.000 & 0.000 & 0.000 & 0.000 & 0.000 \\
\hline $\mathrm{Q}(1)$ & 1724.3 & 783.6 & 2084.1 & 943.2 & 2229.0 \\
\hline Probability & 0.000 & 0.000 & 0.000 & 0.000 & 0.000 \\
\hline $\mathrm{Q}(10)$ & 2767.0 & 2741.4 & 4775.0 & 1974.4 & 3976.5 \\
\hline Probability & 0.000 & 0.000 & 0.000 & 0.000 & 0.000 \\
\hline $\mathrm{Q}(1) \mathrm{sq}$ & 738.38 & 0.471 & 1482.0 & 2.824 & 1642.4 \\
\hline Probability & 0.000 & 0.493 & 0.000 & 0.093 & 0.000 \\
\hline $\mathrm{Q}(10) \mathrm{sq}$ & 1781.8 & 2.327 & 4007.6 & 7.036 & 4115.9 \\
\hline Probability & 0.000 & 0.993 & 0.000 & 0.722 & 0.000 \\
\hline Observations & 3091 & 3091 & 3091 & 3091 & 3091 \\
\hline
\end{tabular}

Notes: Descriptive statistics are calculated for the whole sample of logarithmic returns covering the period 1/11/1999-12/03/2012.

The Jarque-Bera statistic for the normality test is $\chi^{2}(2)$ distributed. $\mathrm{Q}(\mathrm{i})$ and $\mathrm{Q}(\mathrm{i}) \mathrm{sq}$ are the Ljung-Box Q statistics for the returns and squared returns at the i lag. 
Table 2. Backtesting results BDI

\begin{tabular}{|c|c|c|c|c|c|c|c|c|c|c|c|c|c|c|c|}
\hline Panel A: 5\% & $\overline{L L R_{u c}}$ & $L R_{\text {ind }}$ & $L R_{c c}$ & $\overline{L R} R_{\text {muc }}$ & $\overline{D D Q 1 u c}$ & $\overline{D Q^{2}{ }_{\text {uc }}}$ & $\overline{D Q 3 u c}$ & $\overline{D D Q 1_{\text {ind }}}$ & $\overline{D Q Q^{2}{ }_{\text {ind }}}$ & $\overline{D Q 3_{\text {ind }}}$ & $\overline{D Q 1_{c c}}$ & $\overline{D Q 2 c c}$ & $\overline{D Q 3}{ }_{c c}$ & $\overline{D u r_{c c}}$ & Dur $_{\text {ind }}$ \\
\hline Filtered Historical Simulation & 0.820 & 0.866 & 0.961 & 0.126 & 0.820 & 0.822 & 0.814 & 0.863 & 0.248 & 0.497 & 0.960 & 0.526 & 0.650 & 0.947 & 0.750 \\
\hline Historical Simulation-250 & 0.108 & 0.000 & 0.000 & 0.003 & 0.400 & 0.462 & 0.520 & 0.000 & 0.000 & 0.000 & 0.000 & 0.000 & 0.000 & 0.000 & 0.000 \\
\hline Historical Simulation-500 & 0.002 & 0.000 & 0.000 & 0.000 & 0.189 & 0.275 & 0.276 & 0.000 & 0.000 & 0.000 & 0.000 & 0.000 & 0.000 & 0.000 & 0.000 \\
\hline Historical SimulationAll & 0.000 & 0.000 & 0.000 & 0.000 & 0.000 & 0.000 & 0.000 & 0.000 & 0.000 & 0.000 & 0.000 & 0.000 & 0.000 & 0.000 & 0.000 \\
\hline Riskmetrics & 0.841 & 0.000 & 0.000 & 0.813 & 0.919 & 0.924 & 0.927 & 0.000 & 0.000 & 0.000 & 0.000 & 0.000 & 0.000 & 0.000 & 0.000 \\
\hline Variance-Covariance & 0.000 & 0.000 & 0.000 & 0.000 & 0.000 & 0.000 & 0.000 & 0.000 & 0.000 & 0.000 & 0.000 & 0.000 & 0.000 & 0.000 & 0.000 \\
\hline Extreme Value Theory & 0.000 & 0.002 & 0.000 & 0.000 & 0.000 & 0.000 & 0.000 & 0.000 & 0.002 & 0.008 & 0.000 & 0.000 & 0.000 & 0.000 & 0.345 \\
\hline$A R-A R C H(1)-N$ & 0.033 & 0.461 & 0.078 & 0.000 & 0.023 & 0.029 & 0.040 & 0.428 & 0.602 & 0.684 & 0.060 & 0.048 & 0.116 & 0.138 & 0.697 \\
\hline$A R-A R C H(1)-t$ & 0.000 & 0.144 & 0.000 & 0.000 & 0.000 & 0.000 & 0.000 & 0.084 & 0.309 & 0.962 & 0.000 & 0.000 & 0.000 & 0.000 & 0.161 \\
\hline$A R-G A R C H(1,1)-N$ & 0.217 & 0.877 & 0.461 & 0.092 & 0.232 & 0.237 & 0.231 & 0.886 & 0.840 & 0.867 & 0.481 & 0.689 & 0.775 & 0.396 & 0.894 \\
\hline$A R-G A R C H(1,1)-t$ & 0.623 & 0.612 & 0.779 & 0.050 & 0.632 & 0.650 & 0.646 & 0.607 & 0.232 & 0.621 & 0.778 & 0.595 & 0.616 & 0.739 & 0.641 \\
\hline$A R-E G A R C H(1,1)-N$ & 0.350 & 0.428 & 0.472 & 0.422 & 0.349 & 0.364 & 0.358 & 0.498 & 0.977 & 0.726 & 0.522 & 0.618 & 0.700 & 0.570 & 0.956 \\
\hline$A R-E G A R C H(1,1)-t$ & 0.820 & 0.190 & 0.412 & 0.534 & 0.827 & 0.833 & 0.821 & 0.142 & 0.013 & 0.173 & 0.332 & 0.094 & 0.088 & 0.651 & 0.356 \\
\hline Monte Carlo Simulation & 0.729 & 0.661 & 0.855 & 0.064 & 0.735 & 0.754 & 0.752 & 0.655 & 0.117 & 0.436 & 0.853 & 0.323 & 0.357 & 0.720 & 0.513 \\
\hline Combination Mean & 0.432 & 0.000 & 0.000 & 0.078 & 0.527 & 0.585 & 0.587 & 0.000 & 0.000 & 0.000 & 0.000 & 0.000 & 0.000 & 0.002 & 0.001 \\
\hline Combination Median & 0.432 & 0.966 & 0.733 & 0.510 & 0.439 & 0.464 & 0.457 & 0.968 & 0.357 & 0.726 & 0.741 & 0.495 & 0.572 & 0.542 & 0.531 \\
\hline Combination Trim1 & 0.523 & 0.000 & 0.000 & 0.179 & 0.604 & 0.653 & 0.656 & 0.000 & 0.000 & 0.000 & 0.000 & 0.000 & 0.000 & 0.004 & 0.001 \\
\hline Combination Trim2 & 0.278 & 0.011 & 0.021 & 0.580 & 0.340 & 0.388 & 0.384 & 0.004 & 0.000 & 0.002 & 0.008 & 0.001 & 0.002 & 0.019 & 0.011 \\
\hline Panel B: 1\% & $L R_{u c}$ & $L R_{\text {ind }}$ & $L R_{c c}$ & $L R_{m u c}$ & $D Q 1_{u c}$ & $D Q^{2} u c$ & DQ3uc & $D Q 1_{\text {ind }}$ & $D Q^{2}{ }_{\text {ind }}$ & $D Q 3_{\text {ind }}$ & $D Q 1_{c c}$ & $D Q^{2} c c$ & $D Q 3_{c c}$ & $\operatorname{Dur}_{c c}$ & Dur $_{\text {ind }}$ \\
\hline Filtered Historical Simulation & 0.269 & 0.534 & 0.447 & 0.025 & 0.237 & 0.265 & 0.257 & 0.606 & 0.234 & 0.464 & 0.444 & 0.094 & 0.158 & 0.602 & 0.554 \\
\hline Historical Simulation-250 & 0.054 & 0.000 & 0.000 & 0.090 & 0.131 & 0.238 & 0.259 & 0.000 & 0.000 & 0.000 & 0.000 & 0.000 & 0.000 & 0.000 & 0.000 \\
\hline Historical Simulation-500 & 0.015 & 0.000 & 0.000 & 0.000 & 0.242 & 0.263 & 0.326 & 0.000 & 0.000 & 0.000 & 0.000 & 0.000 & 0.000 & 0.000 & 0.000 \\
\hline Historical SimulationAll & 0.000 & 0.000 & 0.000 & 0.000 & 0.000 & 0.005 & 0.010 & 0.000 & 0.000 & 0.000 & 0.000 & 0.000 & 0.000 & 0.000 & 0.000 \\
\hline Riskmetrics & 0.097 & 0.001 & 0.001 & 0.130 & 0.139 & 0.207 & 0.176 & 0.000 & 0.000 & 0.000 & 0.000 & 0.000 & 0.000 & 0.002 & 0.001 \\
\hline Variance-Covariance & 0.000 & 0.000 & 0.000 & 0.000 & 0.000 & 0.000 & 0.000 & 0.000 & 0.000 & 0.000 & 0.000 & 0.000 & 0.000 & 0.000 & 0.000 \\
\hline Extreme Value Theory & 0.003 & 0.445 & 0.010 & 0.004 & 0.001 & 0.002 & 0.001 & 0.206 & 0.078 & 0.376 & 0.002 & 0.002 & 0.004 & 0.027 & 0.822 \\
\hline$A R-A R C H(1)-N$ & 0.000 & 0.709 & 0.000 & 0.000 & 0.000 & 0.000 & 0.000 & 0.501 & 0.161 & 0.064 & 0.000 & 0.000 & 0.000 & 0.000 & 0.279 \\
\hline$A R-A R C H(1)-t$ & 0.000 & 0.210 & 0.000 & 0.000 & 0.000 & 0.000 & 0.000 & 0.142 & 0.467 & 0.424 & 0.000 & 0.000 & 0.000 & 0.000 & 0.548 \\
\hline$A R-G A R C H(1,1)-N$ & 0.015 & 0.359 & 0.033 & 0.021 & 0.008 & 0.011 & 0.009 & 0.131 & 0.036 & 0.220 & 0.008 & 0.008 & 0.012 & 0.094 & 0.936 \\
\hline$A R-G A R C H(1,1)-t$ & 0.269 & 0.534 & 0.447 & 0.083 & 0.237 & 0.265 & 0.257 & 0.606 & 0.234 & 0.464 & 0.444 & 0.094 & 0.158 & 0.602 & 0.554 \\
\hline$A R-E G A R C H(1,1)-N$ & 0.003 & 0.445 & 0.010 & 0.007 & 0.001 & 0.002 & 0.001 & 0.206 & 0.078 & 0.376 & 0.002 & 0.002 & 0.004 & 0.022 & 0.516 \\
\hline$A R-E G A R C H(1,1)-t$ & 0.413 & 0.564 & 0.605 & 0.080 & 0.388 & 0.418 & 0.411 & 0.645 & 0.180 & 0.374 & 0.625 & 0.093 & 0.160 & 0.829 & 0.749 \\
\hline Monte Carlo Simulation & 0.166 & 0.212 & 0.176 & 0.026 & 0.161 & 0.183 & 0.173 & 0.041 & 0.005 & 0.054 & 0.042 & 0.018 & 0.030 & 0.253 & 0.215 \\
\hline Combination Mean & 0.146 & 0.791 & 0.335 & 0.328 & 0.178 & 0.177 & 0.176 & 0.885 & 0.838 & 0.801 & 0.402 & 0.607 & 0.763 & 0.184 & 0.860 \\
\hline Combination Median & 0.930 & 0.658 & 0.903 & 0.387 & 0.929 & 0.938 & 0.940 & 0.756 & 0.068 & 0.164 & 0.949 & 0.038 & 0.076 & 0.881 & 0.767 \\
\hline Combination Trim1 & 0.146 & 0.791 & 0.335 & 0.398 & 0.178 & 0.177 & 0.176 & 0.885 & 0.838 & 0.801 & 0.402 & 0.607 & 0.763 & 0.184 & 0.860 \\
\hline Combination Trim2 & 0.682 & 0.690 & 0.849 & 0.655 & 0.686 & 0.685 & 0.718 & 0.791 & 0.706 & 0.135 & 0.891 & 0.960 & 0.043 & 0.261 & 0.144 \\
\hline
\end{tabular}

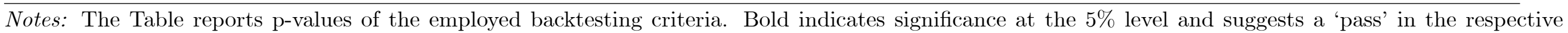
criterion. 
Table 3. Backtesting results BPI

\begin{tabular}{|c|c|c|c|c|c|c|c|c|c|c|c|c|c|c|c|}
\hline Panel A: 5\% & LR uc & $\overline{L L R_{\text {ind }}}$ & $\overline{L L R_{c c}}$ & 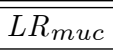 & $\overline{D D Q 1_{u c}}$ & DQ2uc & $\overline{D D Q 3 u c}$ & $D Q 1_{\text {ind }}$ & $D Q 2_{\text {ind }}$ & $D Q 3_{\text {ind }}$ & $\overline{D D Q 1_{c c}}$ & 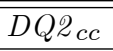 & $\overline{D D Q 3 c c}$ & $\operatorname{Dur}_{c c}$ & 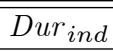 \\
\hline Filtered Historical Simulation & 0.712 & 0.080 & 0.201 & 0.843 & 0.727 & 0.732 & 0.727 & 0.042 & 0.019 & 0.055 & 0.119 & 0.119 & 0.211 & 0.826 & 0.564 \\
\hline Historical Simulation-250 & 0.082 & 0.000 & 0.000 & 0.000 & 0.589 & 0.571 & 0.626 & 0.000 & 0.000 & 0.000 & 0.000 & 0.000 & 0.000 & 0.000 & 0.000 \\
\hline Historical Simulation-500 & 0.002 & 0.000 & 0.000 & 0.000 & 0.226 & 0.249 & 0.257 & 0.000 & 0.000 & 0.000 & 0.000 & 0.000 & 0.000 & 0.000 & 0.000 \\
\hline Historical SimulationAll & 0.000 & 0.000 & 0.000 & 0.000 & 0.001 & 0.001 & 0.001 & 0.000 & 0.000 & 0.000 & 0.000 & 0.000 & 0.000 & 0.000 & 0.000 \\
\hline Riskmetrics & 0.712 & 0.000 & 0.000 & 0.036 & 0.839 & 0.838 & 0.834 & 0.000 & 0.000 & 0.000 & 0.000 & 0.000 & 0.000 & 0.000 & 0.000 \\
\hline Variance-Covariance & 0.000 & 0.000 & 0.000 & 0.000 & 0.013 & 0.015 & 0.014 & 0.000 & 0.000 & 0.000 & 0.000 & 0.000 & 0.000 & 0.000 & 0.000 \\
\hline Extreme Value Theory & 0.000 & 0.001 & 0.000 & 0.000 & 0.000 & 0.000 & 0.000 & 0.000 & 0.000 & 0.000 & 0.000 & 0.000 & 0.000 & 0.000 & 0.433 \\
\hline$A R-A R C H(1)-N$ & 0.354 & 0.872 & 0.643 & 0.350 & 0.343 & 0.345 & 0.376 & 0.866 & 0.844 & 0.137 & 0.631 & 0.768 & 0.165 & 0.186 & 0.096 \\
\hline$A R-A R C H(1)-t$ & 0.000 & 0.369 & 0.001 & 0.000 & 0.000 & 0.000 & 0.000 & 0.300 & 0.655 & 0.295 & 0.000 & 0.000 & 0.001 & 0.002 & 0.319 \\
\hline$A R-G A R C H(1,1)-N$ & 0.432 & 0.065 & 0.134 & 0.771 & 0.471 & 0.472 & 0.486 & 0.042 & 0.172 & 0.134 & 0.093 & 0.190 & 0.269 & 0.640 & 0.804 \\
\hline$A R-G A R C H(1,1)-t$ & 0.932 & 0.166 & 0.381 & 0.871 & 0.935 & 0.933 & 0.928 & 0.122 & 0.033 & 0.067 & 0.301 & 0.206 & 0.334 & 0.696 & 0.396 \\
\hline$A R-E G A R C H(1,1)-N$ & 0.841 & 0.039 & 0.116 & 0.813 & 0.853 & 0.859 & 0.868 & 0.018 & 0.061 & 0.035 & 0.059 & 0.127 & 0.153 & 0.570 & 0.315 \\
\hline$A R-E G A R C H(1,1)-t$ & 0.932 & 0.004 & 0.016 & 0.871 & 0.939 & 0.934 & 0.929 & 0.000 & 0.012 & 0.027 & 0.002 & 0.006 & 0.015 & 0.401 & 0.177 \\
\hline Monte Carlo Simulation & 0.820 & 0.067 & 0.183 & 0.716 & 0.830 & 0.829 & 0.824 & 0.034 & 0.045 & 0.088 & 0.104 & 0.173 & 0.289 & 0.620 & 0.330 \\
\hline Combination Mean & 0.030 & 0.000 & 0.000 & 0.067 & 0.084 & 0.125 & 0.169 & 0.000 & 0.000 & 0.000 & 0.000 & 0.000 & 0.000 & 0.000 & 0.000 \\
\hline Combination Median & 0.045 & 0.220 & 0.063 & 0.113 & 0.067 & 0.081 & 0.096 & 0.226 & 0.094 & 0.045 & 0.076 & 0.091 & 0.105 & 0.019 & 0.064 \\
\hline Combination Trim1 & 0.013 & 0.001 & 0.000 & 0.053 & 0.045 & 0.071 & 0.113 & 0.000 & 0.000 & 0.000 & 0.000 & 0.000 & 0.000 & 0.000 & 0.000 \\
\hline Combination Trim2 & 0.013 & 0.001 & 0.000 & 0.010 & 0.045 & 0.071 & 0.101 & 0.000 & 0.000 & 0.000 & 0.000 & 0.000 & 0.000 & 0.000 & 0.001 \\
\hline Panel B: 1\% & $L R_{u c}$ & $L R_{\text {ind }}$ & $L R_{c c}$ & $L R_{m u c}$ & $D Q 1_{u c}$ & $D Q^{2} u c$ & $D Q 3 u c$ & $D Q 1_{\text {ind }}$ & $D Q 2_{\text {ind }}$ & $D Q 3_{\text {ind }}$ & $D Q 1_{c c}$ & $D Q 2 c c$ & $D Q 3_{c c}$ & $\operatorname{Dur}_{c c}$ & Dur ${ }_{i n d}$ \\
\hline Filtered Historical Simulation & 0.413 & 0.564 & 0.605 & 0.641 & 0.388 & 0.380 & 0.410 & 0.645 & 0.511 & 0.407 & 0.625 & 0.761 & 0.158 & 0.832 & 0.759 \\
\hline Historical Simulation-250 & 0.097 & 0.000 & 0.000 & 0.007 & 0.306 & 0.380 & 0.344 & 0.000 & 0.000 & 0.000 & 0.000 & 0.000 & 0.000 & 0.000 & 0.000 \\
\hline Historical Simulation-500 & 0.054 & 0.000 & 0.000 & 0.002 & 0.314 & 0.282 & 0.333 & 0.000 & 0.000 & 0.000 & 0.000 & 0.000 & 0.000 & 0.000 & 0.000 \\
\hline Historical SimulationAll & 0.000 & 0.000 & 0.000 & 0.000 & 0.098 & 0.054 & 0.083 & 0.000 & 0.000 & 0.000 & 0.000 & 0.000 & 0.000 & 0.000 & 0.000 \\
\hline Riskmetrics & 0.007 & 0.000 & 0.000 & 0.017 & 0.063 & 0.074 & 0.092 & 0.000 & 0.000 & 0.000 & 0.000 & 0.000 & 0.000 & 0.000 & 0.000 \\
\hline Variance-Covariance & 0.000 & 0.000 & 0.000 & 0.000 & 0.000 & 0.000 & 0.000 & 0.000 & 0.000 & 0.000 & 0.000 & 0.000 & 0.000 & 0.000 & 0.000 \\
\hline Extreme Value Theory & 0.007 & 0.373 & 0.018 & 0.021 & 0.002 & 0.003 & 0.004 & 0.377 & 0.720 & 0.266 & 0.007 & 0.008 & 0.007 & 0.044 & 0.554 \\
\hline$A R-A R C H(1)-N$ & 0.000 & 0.264 & 0.000 & 0.000 & 0.000 & 0.000 & 0.000 & 0.216 & 0.752 & 0.825 & 0.000 & 0.000 & 0.000 & 0.001 & 0.240 \\
\hline$A R-A R C H(1)-t$ & 0.097 & 0.477 & 0.196 & 0.024 & 0.069 & 0.064 & 0.060 & 0.528 & 0.368 & 0.266 & 0.164 & 0.257 & 0.345 & 0.037 & 0.030 \\
\hline$A R-G A R C H(1,1)-N$ & 0.054 & 0.450 & 0.118 & 0.125 & 0.032 & 0.040 & 0.050 & 0.490 & 0.443 & 0.097 & 0.086 & 0.046 & 0.023 & 0.254 & 0.873 \\
\hline$A R-G A R C H(1,1)-t$ & 0.930 & 0.658 & 0.903 & 0.664 & 0.929 & 0.931 & 0.940 & 0.756 & 0.659 & 0.188 & 0.949 & 0.977 & 0.076 & 0.899 & 0.827 \\
\hline$A R-E G A R C H(1,1)-N$ & 0.097 & 0.477 & 0.196 & 0.197 & 0.069 & 0.083 & 0.099 & 0.528 & 0.365 & 0.064 & 0.164 & 0.066 & 0.026 & 0.194 & 0.240 \\
\hline$A R-E G A R C H(1,1)-t$ & 0.599 & 0.594 & 0.756 & 0.768 & 0.585 & 0.579 & 0.604 & 0.683 & 0.560 & 0.324 & 0.795 & 0.889 & 0.142 & 0.972 & 0.927 \\
\hline Monte Carlo Simulation & 0.930 & 0.658 & 0.903 & 0.998 & 0.929 & 0.931 & 0.933 & 0.756 & 0.659 & 0.586 & 0.949 & 0.977 & 0.990 & 0.625 & 0.379 \\
\hline Combination Mean & 0.457 & 0.048 & 0.107 & 0.679 & 0.529 & 0.575 & 0.613 & 0.001 & 0.000 & 0.000 & 0.003 & 0.000 & 0.000 & 0.081 & 0.050 \\
\hline Combination Median & 0.457 & 0.723 & 0.712 & 0.530 & 0.471 & 0.470 & 0.468 & 0.824 & 0.752 & 0.698 & 0.756 & 0.895 & 0.957 & 0.554 & 0.975 \\
\hline Combination Trim1 & 0.007 & 0.895 & 0.027 & 0.037 & 0.023 & 0.024 & 0.135 & 0.959 & 0.943 & 0.001 & 0.074 & 0.157 & 0.000 & 0.001 & 0.025 \\
\hline Combination Trim2 & 0.457 & 0.723 & 0.712 & 0.679 & 0.471 & 0.470 & 0.526 & 0.824 & 0.752 & 0.092 & 0.756 & 0.895 & 0.019 & 0.290 & 0.255 \\
\hline
\end{tabular}

Notes: See Table 2. 
Table 4. Backtesting results BCI

\begin{tabular}{|c|c|c|c|c|c|c|c|c|c|c|c|c|c|c|c|}
\hline Panel A: 5\% & 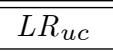 & $\overline{L L R_{\text {ind }}}$ & $\overline{L L R_{c c}}$ & 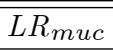 & $\overline{D D Q 1_{u c}}$ & DQ2uc & $\overline{D D Q 3 u c}$ & $D Q 1_{\text {ind }}$ & $D Q 2_{\text {ind }}$ & $D Q 3_{\text {ind }}$ & $\overline{D D Q 1_{c c}}$ & 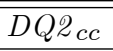 & $\overline{D D Q 3 c c}$ & $\operatorname{Dur}_{c c}$ & 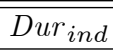 \\
\hline Filtered Historical Simulation & 0.431 & 0.630 & 0.653 & 0.158 & 0.430 & 0.433 & 0.436 & 0.600 & 0.463 & 0.374 & 0.632 & 0.755 & 0.834 & 0.818 & 0.967 \\
\hline Historical Simulation-250 & 0.516 & 0.000 & 0.000 & 0.087 & 0.682 & 0.688 & 0.685 & 0.000 & 0.000 & 0.000 & 0.000 & 0.000 & 0.000 & 0.000 & 0.000 \\
\hline Historical Simulation-500 & 0.061 & 0.000 & 0.000 & 0.001 & 0.251 & 0.275 & 0.288 & 0.000 & 0.000 & 0.000 & 0.000 & 0.000 & 0.000 & 0.000 & 0.000 \\
\hline Historical SimulationAll & 0.000 & 0.000 & 0.000 & 0.000 & 0.000 & 0.000 & 0.000 & 0.000 & 0.000 & 0.000 & 0.000 & 0.000 & 0.000 & 0.000 & 0.000 \\
\hline Riskmetrics & 0.278 & 0.000 & 0.000 & 0.469 & 0.497 & 0.544 & 0.511 & 0.000 & 0.000 & 0.000 & 0.000 & 0.000 & 0.000 & 0.000 & 0.000 \\
\hline Variance-Covariance & 0.000 & 0.000 & 0.000 & 0.000 & 0.000 & 0.000 & 0.000 & 0.000 & 0.000 & 0.000 & 0.000 & 0.000 & 0.000 & 0.000 & 0.000 \\
\hline Extreme Value Theory & 0.000 & 0.150 & 0.000 & 0.000 & 0.000 & 0.000 & 0.000 & 0.047 & 0.090 & 0.285 & 0.000 & 0.000 & 0.000 & 0.000 & 0.528 \\
\hline$A R-A R C H(1)-N$ & 0.004 & 0.562 & 0.012 & 0.000 & 0.002 & 0.003 & 0.003 & 0.510 & 0.519 & 0.618 & 0.007 & 0.006 & 0.014 & 0.023 & 0.646 \\
\hline$A R-A R C H(1)-t$ & 0.000 & 0.487 & 0.000 & 0.000 & 0.000 & 0.000 & 0.000 & 0.386 & 0.834 & 0.571 & 0.000 & 0.000 & 0.000 & 0.000 & 0.800 \\
\hline$A R-G A R C H(1,1)-N$ & 0.278 & 0.930 & 0.553 & 0.219 & 0.291 & 0.308 & 0.324 & 0.934 & 0.539 & 0.343 & 0.569 & 0.629 & 0.675 & 0.481 & 0.925 \\
\hline$A R-G A R C H(1,1)-t$ & 0.610 & 0.972 & 0.878 & 0.149 & 0.607 & 0.602 & 0.604 & 0.971 & 0.961 & 0.672 & 0.875 & 0.965 & 0.948 & 0.928 & 0.886 \\
\hline$A R-E G A R C H(1,1)-N$ & 0.841 & 0.329 & 0.609 & 0.429 & 0.846 & 0.850 & 0.860 & 0.297 & 0.600 & 0.300 & 0.569 & 0.747 & 0.672 & 0.944 & 0.971 \\
\hline$A R-E G A R C H(1,1)-t$ & 0.181 & 0.219 & 0.192 & 0.131 & 0.188 & 0.188 & 0.197 & 0.149 & 0.248 & 0.158 & 0.138 & 0.260 & 0.320 & 0.444 & 0.609 \\
\hline Monte Carlo Simulation & 0.354 & 0.343 & 0.416 & 0.092 & 0.361 & 0.363 & 0.365 & 0.284 & 0.290 & 0.268 & 0.361 & 0.527 & 0.661 & 0.724 & 0.811 \\
\hline Combination Mean & 0.165 & 0.030 & 0.037 & 0.434 & 0.217 & 0.256 & 0.281 & 0.018 & 0.001 & 0.001 & 0.024 & 0.007 & 0.008 & 0.114 & 0.148 \\
\hline Combination Median & 0.623 & 0.091 & 0.213 & 0.681 & 0.648 & 0.656 & 0.666 & 0.061 & 0.102 & 0.107 & 0.154 & 0.274 & 0.395 & 0.732 & 0.626 \\
\hline Combination Trim1 & 0.217 & 0.037 & 0.053 & 0.526 & 0.268 & 0.307 & 0.331 & 0.022 & 0.002 & 0.001 & 0.035 & 0.011 & 0.013 & 0.172 & 0.195 \\
\hline Combination Trim2 & 0.350 & 0.054 & 0.101 & 0.675 & 0.395 & 0.430 & 0.451 & 0.034 & 0.004 & 0.002 & 0.070 & 0.027 & 0.033 & 0.273 & 0.225 \\
\hline Panel B: 1\% & $L R_{u c}$ & $L R_{\text {ind }}$ & $L R_{c c}$ & $L R_{m u c}$ & $D Q 1_{u c}$ & $D Q^{2} u c$ & $D Q 3 u c$ & $D Q 1_{\text {ind }}$ & $D Q 2_{\text {ind }}$ & $D Q 3_{\text {ind }}$ & $D Q 1_{c c}$ & $D Q 2 c c$ & $D Q 3_{c c}$ & $\operatorname{Dur}_{c c}$ & Dur ${ }_{i n d}$ \\
\hline Filtered Historical Simulation & 0.166 & 0.505 & 0.307 & 0.077 & 0.134 & 0.127 & 0.147 & 0.567 & 0.414 & 0.600 & 0.284 & 0.413 & 0.137 & 0.534 & 0.830 \\
\hline Historical Simulation-250 & 0.269 & 0.012 & 0.023 & 0.207 & 0.311 & 0.410 & 0.415 & 0.000 & 0.000 & 0.000 & 0.000 & 0.000 & 0.000 & 0.017 & 0.006 \\
\hline Historical Simulation-500 & 0.166 & 0.001 & 0.001 & 0.335 & 0.230 & 0.353 & 0.338 & 0.000 & 0.000 & 0.000 & 0.000 & 0.000 & 0.000 & 0.000 & 0.000 \\
\hline Historical SimulationAll & 0.000 & 0.000 & 0.000 & 0.000 & 0.000 & 0.000 & 0.000 & 0.000 & 0.000 & 0.000 & 0.000 & 0.000 & 0.000 & 0.000 & 0.000 \\
\hline Riskmetrics & 0.269 & 0.000 & 0.001 & 0.406 & 0.353 & 0.361 & 0.381 & 0.000 & 0.000 & 0.000 & 0.000 & 0.000 & 0.000 & 0.005 & 0.002 \\
\hline Variance-Covariance & 0.000 & 0.000 & 0.000 & 0.000 & 0.000 & 0.000 & 0.000 & 0.000 & 0.000 & 0.000 & 0.000 & 0.000 & 0.000 & 0.000 & 0.000 \\
\hline Extreme Value Theory & 0.001 & 0.491 & 0.005 & 0.000 & 0.000 & 0.000 & 0.000 & 0.252 & 0.931 & 0.444 & 0.001 & 0.001 & 0.002 & 0.014 & 0.928 \\
\hline$A R-A R C H(1)-N$ & 0.000 & 0.038 & 0.000 & 0.000 & 0.000 & 0.000 & 0.000 & 0.002 & 0.334 & 0.334 & 0.000 & 0.000 & 0.000 & 0.000 & 0.508 \\
\hline$A R-A R C H(1)-t$ & 0.000 & 0.227 & 0.000 & 0.000 & 0.000 & 0.000 & 0.000 & 0.165 & 0.555 & 0.891 & 0.000 & 0.000 & 0.000 & 0.000 & 0.927 \\
\hline$A R-G A R C H(1,1)-N$ & 0.007 & 0.401 & 0.019 & 0.003 & 0.003 & 0.003 & 0.004 & 0.166 & 0.727 & 0.263 & 0.004 & 0.007 & 0.007 & 0.046 & 0.605 \\
\hline$A R-G A R C H(1,1)-t$ & 0.599 & 0.594 & 0.756 & 0.213 & 0.585 & 0.579 & 0.572 & 0.683 & 0.560 & 0.473 & 0.795 & 0.889 & 0.937 & 0.277 & 0.113 \\
\hline$A R-E G A R C H(1,1)-N$ & 0.015 & 0.398 & 0.035 & 0.004 & 0.005 & 0.005 & 0.006 & 0.414 & 0.243 & 0.939 & 0.017 & 0.031 & 0.026 & 0.088 & 0.723 \\
\hline$A R-E G A R C H(1,1)-t$ & 0.269 & 0.534 & 0.447 & 0.083 & 0.237 & 0.230 & 0.222 & 0.606 & 0.462 & 0.364 & 0.444 & 0.592 & 0.699 & 0.540 & 0.450 \\
\hline Monte Carlo Simulation & 0.413 & 0.564 & 0.605 & 0.452 & 0.388 & 0.380 & 0.372 & 0.645 & 0.511 & 0.417 & 0.625 & 0.761 & 0.845 & 0.358 & 0.182 \\
\hline Combination Mean & 0.823 & 0.626 & 0.866 & 0.827 & 0.820 & 0.815 & 0.810 & 0.720 & 0.610 & 0.529 & 0.914 & 0.958 & 0.978 & 0.882 & 0.622 \\
\hline Combination Median & 0.269 & 0.534 & 0.447 & 0.485 & 0.237 & 0.230 & 0.257 & 0.606 & 0.462 & 0.499 & 0.444 & 0.592 & 0.156 & 0.702 & 0.833 \\
\hline Combination Trim1 & 0.682 & 0.690 & 0.849 & 0.951 & 0.686 & 0.685 & 0.685 & 0.791 & 0.706 & 0.642 & 0.891 & 0.960 & 0.985 & 0.760 & 0.997 \\
\hline Combination Trim2 & 0.599 & 0.594 & 0.756 & 0.775 & 0.585 & 0.579 & 0.572 & 0.683 & 0.560 & 0.473 & 0.795 & 0.889 & 0.937 & 0.950 & 0.819 \\
\hline
\end{tabular}

Notes: See Table 2. 
Table 5. Backtesting results BDTI

\begin{tabular}{|c|c|c|c|c|c|c|c|c|c|c|c|c|c|c|c|}
\hline Panel A: 5\% & LR uc & $\overline{L L R_{\text {ind }}}$ & $\overline{L L R_{c c}}$ & 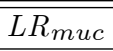 & $D Q 1 u c$ & DQ2uc & $\overline{D D Q 3 u c}$ & $D Q 1_{\text {ind }}$ & $D Q 2_{\text {ind }}$ & $D Q 3_{\text {ind }}$ & $\overline{D D Q 1_{c c}}$ & 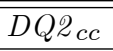 & $\overline{D D Q 3 c c}$ & $\operatorname{Dur}_{c c}$ & 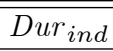 \\
\hline Filtered Historical Simulation & 0.045 & 0.220 & 0.063 & 0.158 & 0.067 & 0.073 & 0.064 & 0.226 & 0.251 & 0.745 & 0.076 & 0.153 & 0.167 & 0.104 & 0.815 \\
\hline Historical Simulation-250 & 0.030 & 0.000 & 0.000 & 0.036 & 0.120 & 0.166 & 0.203 & 0.000 & 0.000 & 0.000 & 0.000 & 0.000 & 0.000 & 0.000 & 0.000 \\
\hline Historical Simulation-500 & 0.008 & 0.000 & 0.000 & 0.017 & 0.070 & 0.098 & 0.111 & 0.000 & 0.000 & 0.000 & 0.000 & 0.000 & 0.000 & 0.000 & 0.000 \\
\hline Historical SimulationAll & 0.003 & 0.000 & 0.000 & 0.017 & 0.050 & 0.074 & 0.086 & 0.000 & 0.000 & 0.000 & 0.000 & 0.000 & 0.000 & 0.000 & 0.000 \\
\hline Riskmetrics & 0.000 & 0.000 & 0.000 & 0.000 & 0.004 & 0.010 & 0.010 & 0.000 & 0.000 & 0.000 & 0.000 & 0.000 & 0.000 & 0.000 & 0.005 \\
\hline Variance-Covariance & 0.000 & 0.000 & 0.000 & 0.000 & 0.011 & 0.022 & 0.034 & 0.000 & 0.000 & 0.000 & 0.000 & 0.000 & 0.000 & 0.000 & 0.000 \\
\hline Extreme Value Theory & 0.000 & 0.120 & 0.000 & 0.000 & 0.000 & 0.000 & 0.000 & 0.033 & 0.003 & 0.002 & 0.000 & 0.000 & 0.000 & 0.000 & 0.438 \\
\hline$A R-A R C H(1)-N$ & 0.000 & 0.211 & 0.000 & 0.000 & 0.000 & 0.000 & 0.000 & 0.351 & 0.644 & 0.806 & 0.000 & 0.000 & 0.000 & 0.000 & 0.021 \\
\hline$A R-A R C H(1)-t$ & 0.000 & 0.789 & 0.001 & 0.002 & 0.001 & 0.001 & 0.002 & 0.835 & 0.770 & 0.722 & 0.004 & 0.010 & 0.024 & 0.000 & 0.230 \\
\hline$A R-G A R C H(1,1)-N$ & 0.000 & 0.012 & 0.000 & 0.000 & 0.000 & 0.000 & 0.000 & 0.025 & 0.184 & 0.306 & 0.000 & 0.000 & 0.000 & 0.000 & 0.010 \\
\hline$A R-G A R C H(1,1)-t$ & 0.001 & 0.252 & 0.001 & 0.003 & 0.002 & 0.003 & 0.000 & 0.307 & 0.415 & 0.793 & 0.003 & 0.010 & 0.019 & 0.001 & 0.348 \\
\hline$A R-E G A R C H(1,1)-N$ & 0.000 & 0.022 & 0.000 & 0.000 & 0.000 & 0.000 & 0.000 & 0.043 & 0.052 & 0.166 & 0.000 & 0.000 & 0.000 & 0.000 & 0.018 \\
\hline$A R-E G A R C H(1,1)-t$ & 0.013 & 0.482 & 0.036 & 0.060 & 0.022 & 0.022 & 0.019 & 0.524 & 0.735 & 0.794 & 0.052 & 0.116 & 0.149 & 0.033 & 0.722 \\
\hline Monte Carlo Simulation & 0.000 & 0.789 & 0.001 & 0.002 & 0.001 & 0.001 & 0.001 & 0.835 & 0.744 & 0.514 & 0.004 & 0.009 & 0.017 & 0.001 & 0.687 \\
\hline Combination Mean & 0.000 & 0.359 & 0.000 & 0.000 & 0.000 & 0.000 & 0.000 & 0.491 & 0.090 & 0.082 & 0.000 & 0.000 & 0.000 & 0.000 & 0.134 \\
\hline Combination Median & 0.000 & 0.058 & 0.000 & 0.000 & 0.000 & 0.000 & 0.000 & 0.095 & 0.118 & 0.303 & 0.000 & 0.000 & 0.000 & 0.000 & 0.031 \\
\hline Combination Trim1 & 0.000 & 0.401 & 0.000 & 0.000 & 0.000 & 0.000 & 0.000 & 0.527 & 0.109 & 0.102 & 0.000 & 0.000 & 0.000 & 0.000 & 0.100 \\
\hline Combination Trim2 & 0.000 & 0.445 & 0.000 & 0.000 & 0.000 & 0.000 & 0.000 & 0.563 & 0.131 & 0.124 & 0.000 & 0.000 & 0.000 & 0.000 & 0.177 \\
\hline Panel B: 1\% & $L R_{u c}$ & $L R_{\text {ind }}$ & $L R_{c c}$ & $L R_{m u c}$ & $D Q 1 u c$ & $D Q^{2} u c$ & $D Q 3 u c$ & $D Q 1_{\text {ind }}$ & $D Q 2_{\text {ind }}$ & $D Q 3_{\text {ind }}$ & $D Q 1_{c c}$ & $D Q 2 c c$ & $D Q 3_{c c}$ & $\operatorname{Dur}_{c c}$ & Dur ${ }_{i n d}$ \\
\hline Filtered Historical Simulation & 0.146 & 0.791 & 0.335 & 0.127 & 0.178 & 0.177 & 0.176 & 0.885 & 0.838 & 0.801 & 0.402 & 0.607 & 0.763 & 0.173 & 0.695 \\
\hline Historical Simulation-250 & 0.275 & 0.757 & 0.526 & 0.272 & 0.301 & 0.299 & 0.297 & 0.856 & 0.796 & 0.751 & 0.580 & 0.773 & 0.887 & 0.279 & 0.501 \\
\hline Historical Simulation-500 & 0.275 & 0.757 & 0.526 & 0.272 & 0.301 & 0.458 & 0.458 & 0.856 & 0.000 & 0.000 & 0.580 & 0.000 & 0.000 & 0.048 & 0.046 \\
\hline Historical SimulationAll & 0.275 & 0.757 & 0.526 & 0.272 & 0.301 & 0.458 & 0.458 & 0.856 & 0.000 & 0.000 & 0.580 & 0.000 & 0.000 & 0.048 & 0.046 \\
\hline Riskmetrics & 0.275 & 0.757 & 0.526 & 0.272 & 0.301 & 0.299 & 0.297 & 0.856 & 0.796 & 0.751 & 0.580 & 0.773 & 0.887 & 0.129 & 0.157 \\
\hline Variance-Covariance & 0.275 & 0.757 & 0.526 & 0.272 & 0.301 & 0.458 & 0.458 & 0.856 & 0.000 & 0.000 & 0.580 & 0.000 & 0.000 & 0.048 & 0.046 \\
\hline Extreme Value Theory & 0.680 & 0.690 & 0.848 & 0.680 & 0.683 & 0.683 & 0.683 & 0.791 & 0.706 & 0.643 & 0.890 & 0.959 & 0.985 & 0.478 & 0.336 \\
\hline$A R-A R C H(1)-N$ & 0.066 & 0.825 & 0.179 & 0.007 & 0.097 & 0.096 & 0.096 & 0.913 & 0.877 & 0.849 & 0.252 & 0.430 & 0.597 & 0.052 & 0.344 \\
\hline$A R-A R C H(1)-t$ & 0.146 & 0.791 & 0.335 & 0.127 & 0.178 & 0.177 & 0.176 & 0.885 & 0.838 & 0.801 & 0.402 & 0.607 & 0.763 & 0.187 & 0.982 \\
\hline$A R-G A R C H(1,1)-N$ & 0.146 & 0.791 & 0.335 & 0.127 & 0.178 & 0.177 & 0.176 & 0.885 & 0.838 & 0.801 & 0.402 & 0.607 & 0.763 & 0.173 & 0.695 \\
\hline$A R-G A R C H(1,1)-t$ & 0.066 & 0.825 & 0.180 & 0.043 & 0.097 & 0.097 & 0.097 & 0.913 & 0.876 & 0.848 & 0.253 & 0.431 & 0.599 & 0.052 & 0.345 \\
\hline$A R-E G A R C H(1,1)-N$ & 0.457 & 0.723 & 0.712 & 0.464 & 0.471 & 0.527 & 0.526 & 0.824 & 0.027 & 0.075 & 0.756 & 0.008 & 0.019 & 0.279 & 0.241 \\
\hline$A R-E G A R C H(1,1)-t$ & 0.025 & 0.860 & 0.079 & 0.010 & 0.049 & 0.050 & 0.050 & 0.938 & 0.911 & 0.891 & 0.144 & 0.275 & 0.423 & 0.011 & 0.168 \\
\hline Monte Carlo Simulation & 0.066 & 0.825 & 0.180 & 0.043 & 0.097 & 0.097 & 0.097 & 0.913 & 0.876 & 0.848 & 0.253 & 0.431 & 0.599 & 0.052 & 0.345 \\
\hline Combination Mean & 0.066 & 0.825 & 0.180 & 0.043 & 0.097 & 0.097 & 0.097 & 0.913 & 0.876 & 0.848 & 0.253 & 0.431 & 0.599 & 0.052 & 0.345 \\
\hline Combination Median & 0.066 & 0.825 & 0.180 & 0.043 & 0.097 & 0.097 & 0.097 & 0.913 & 0.876 & 0.848 & 0.253 & 0.431 & 0.599 & 0.052 & 0.345 \\
\hline Combination Trim1 & 0.007 & 0.895 & 0.027 & 0.539 & 0.023 & 0.024 & 0.025 & 0.959 & 0.943 & 0.929 & 0.074 & 0.157 & 0.268 & 0.001 & 0.050 \\
\hline Combination Trim2 & 0.066 & 0.825 & 0.180 & 0.043 & 0.097 & 0.097 & 0.097 & 0.913 & 0.876 & 0.848 & 0.253 & 0.431 & 0.599 & 0.052 & 0.345 \\
\hline
\end{tabular}

Notes: See Table 2. 
Table 6. Backtesting results BCTI

\begin{tabular}{|c|c|c|c|c|c|c|c|c|c|c|c|c|c|c|c|}
\hline Panel A: 5\% & LR uc & $L R_{\text {ind }}$ & $\overline{L L R_{c c}}$ & 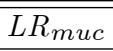 & $D Q 1 u c$ & DQ2uc & $\overline{D D Q 3 u c}$ & $D Q 1_{\text {ind }}$ & $D Q 2_{\text {ind }}$ & $D Q 3_{\text {ind }}$ & $\overline{D D Q 1_{c c}}$ & 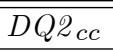 & $\overline{D D Q 3 c c}$ & $\operatorname{Dur}_{c c}$ & 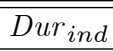 \\
\hline Filtered Historical Simulation & 0.432 & 0.395 & 0.511 & 0.665 & 0.429 & 0.443 & 0.426 & 0.464 & 0.952 & 0.394 & 0.567 & 0.676 & 0.483 & 0.325 & 0.234 \\
\hline Historical Simulation-250 & 0.431 & 0.000 & 0.000 & 0.606 & 0.563 & 0.603 & 0.592 & 0.000 & 0.000 & 0.000 & 0.000 & 0.000 & 0.000 & 0.000 & 0.000 \\
\hline Historical Simulation-500 & 0.932 & 0.000 & 0.000 & 0.848 & 0.915 & 0.907 & 0.904 & 0.000 & 0.000 & 0.000 & 0.000 & 0.000 & 0.000 & 0.000 & 0.000 \\
\hline Historical SimulationAll & 0.013 & 0.000 & 0.000 & 0.060 & 0.097 & 0.150 & 0.163 & 0.000 & 0.000 & 0.000 & 0.000 & 0.000 & 0.000 & 0.000 & 0.000 \\
\hline Riskmetrics & 0.000 & 0.027 & 0.000 & 0.000 & 0.001 & 0.002 & 0.002 & 0.033 & 0.023 & 0.141 & 0.000 & 0.000 & 0.001 & 0.000 & 0.081 \\
\hline Variance-Covariance & 0.000 & 0.037 & 0.000 & 0.000 & 0.000 & 0.000 & 0.001 & 0.066 & 0.001 & 0.004 & 0.000 & 0.000 & 0.000 & 0.000 & 0.001 \\
\hline Extreme Value Theory & 0.000 & 0.772 & 0.000 & 0.000 & 0.000 & 0.000 & 0.000 & 0.701 & 0.570 & 0.303 & 0.000 & 0.000 & 0.000 & 0.000 & 0.603 \\
\hline$A R-A R C H(1)-N$ & 0.000 & 0.534 & 0.000 & 0.000 & 0.000 & 0.000 & 0.000 & 0.814 & 0.587 & 0.738 & 0.000 & 0.000 & 0.000 & 0.000 & 0.051 \\
\hline$A R-A R C H(1)-t$ & 0.008 & 0.902 & 0.030 & 0.041 & 0.013 & 0.024 & 0.020 & 0.921 & 0.119 & 0.411 & 0.045 & 0.009 & 0.016 & 0.019 & 0.522 \\
\hline$A R-G A R C H(1,1)-N$ & 0.000 & 0.564 & 0.000 & 0.000 & 0.000 & 0.000 & 0.000 & 0.833 & 0.764 & 0.711 & 0.000 & 0.000 & 0.000 & 0.000 & 0.091 \\
\hline$A R-G A R C H(1,1)-t$ & 0.020 & 0.803 & 0.065 & 0.070 & 0.027 & 0.027 & 0.022 & 0.839 & 0.772 & 0.418 & 0.086 & 0.178 & 0.210 & 0.040 & 0.472 \\
\hline$A R-E G A R C H(1,1)-N$ & 0.000 & 0.423 & 0.000 & 0.000 & 0.000 & 0.000 & 0.000 & 0.731 & 0.774 & 0.955 & 0.000 & 0.000 & 0.000 & 0.000 & 0.287 \\
\hline$A R-E G A R C H(1,1)-t$ & 0.020 & 0.803 & 0.065 & 0.086 & 0.027 & 0.031 & 0.027 & 0.839 & 0.792 & 0.733 & 0.086 & 0.158 & 0.190 & 0.048 & 0.680 \\
\hline Monte Carlo Simulation & 0.030 & 0.756 & 0.092 & 0.120 & 0.038 & 0.043 & 0.037 & 0.799 & 0.855 & 0.665 & 0.116 & 0.208 & 0.235 & 0.069 & 0.657 \\
\hline Combination Mean & 0.000 & 0.423 & 0.000 & 0.000 & 0.000 & 0.000 & 0.000 & 0.731 & 0.287 & 0.460 & 0.000 & 0.000 & 0.000 & 0.000 & 0.074 \\
\hline Combination Median & 0.000 & 0.491 & 0.000 & 0.000 & 0.000 & 0.000 & 0.000 & 0.601 & 0.465 & 0.744 & 0.000 & 0.000 & 0.001 & 0.000 & 0.465 \\
\hline Combination Trim1 & 0.000 & 0.349 & 0.000 & 0.000 & 0.000 & 0.000 & 0.000 & 0.664 & 0.409 & 0.630 & 0.000 & 0.000 & 0.000 & 0.000 & 0.171 \\
\hline Combination Trim2 & 0.000 & 0.491 & 0.000 & 0.000 & 0.000 & 0.000 & 0.000 & 0.601 & 0.155 & 0.370 & 0.000 & 0.000 & 0.000 & 0.000 & 0.071 \\
\hline Panel B: 1\% & $L R_{u c}$ & $L R_{\text {ind }}$ & $L R_{c c}$ & $L R_{m u c}$ & $D Q 1 u c$ & $D Q^{2} u c$ & $D Q 3 u c$ & $D Q 1_{\text {ind }}$ & $D Q 2_{\text {ind }}$ & $D Q 3_{\text {ind }}$ & $D Q 1_{c c}$ & $D Q 2 c c$ & $D Q 3_{c c}$ & $\operatorname{Dur}_{c c}$ & Dur ${ }_{i n d}$ \\
\hline Filtered Historical Simulation & 0.930 & 0.658 & 0.903 & 0.126 & 0.929 & 0.931 & 0.933 & 0.756 & 0.659 & 0.586 & 0.949 & 0.977 & 0.990 & 0.591 & 0.347 \\
\hline Historical Simulation-250 & 0.054 & 0.029 & 0.014 & 0.090 & 0.059 & 0.110 & 0.093 & 0.000 & 0.000 & 0.000 & 0.000 & 0.000 & 0.000 & 0.003 & 0.003 \\
\hline Historical Simulation-500 & 0.269 & 0.012 & 0.023 & 0.377 & 0.311 & 0.331 & 0.352 & 0.000 & 0.000 & 0.000 & 0.000 & 0.000 & 0.000 & 0.031 & 0.012 \\
\hline Historical SimulationAll & 0.682 & 0.064 & 0.165 & 0.274 & 0.719 & 0.746 & 0.741 & 0.002 & 0.000 & 0.002 & 0.008 & 0.001 & 0.001 & 0.230 & 0.122 \\
\hline Riskmetrics & 0.682 & 0.690 & 0.849 & 0.655 & 0.686 & 0.685 & 0.685 & 0.791 & 0.706 & 0.642 & 0.891 & 0.960 & 0.985 & 0.622 & 0.526 \\
\hline Variance-Covariance & 0.146 & 0.024 & 0.027 & 0.026 & 0.261 & 0.335 & 0.311 & 0.000 & 0.000 & 0.000 & 0.000 & 0.000 & 0.000 & 0.027 & 0.048 \\
\hline Extreme Value Theory & 0.269 & 0.534 & 0.447 & 0.207 & 0.237 & 0.230 & 0.222 & 0.606 & 0.462 & 0.364 & 0.444 & 0.592 & 0.699 & 0.154 & 0.079 \\
\hline$A R-A R C H(1)-N$ & 0.066 & 0.825 & 0.180 & 0.006 & 0.097 & 0.097 & 0.097 & 0.913 & 0.876 & 0.848 & 0.253 & 0.431 & 0.599 & 0.062 & 0.455 \\
\hline$A R-A R C H(1)-t$ & 0.146 & 0.791 & 0.335 & 0.026 & 0.178 & 0.177 & 0.176 & 0.885 & 0.838 & 0.801 & 0.402 & 0.607 & 0.763 & 0.175 & 0.717 \\
\hline$A R-G A R C H(1,1)-N$ & 0.275 & 0.757 & 0.526 & 0.075 & 0.301 & 0.299 & 0.297 & 0.856 & 0.796 & 0.751 & 0.580 & 0.773 & 0.887 & 0.348 & 0.906 \\
\hline$A R-G A R C H(1,1)-t$ & 0.066 & 0.825 & 0.180 & 0.006 & 0.097 & 0.097 & 0.097 & 0.913 & 0.876 & 0.848 & 0.253 & 0.431 & 0.599 & 0.057 & 0.392 \\
\hline$A R-E G A R C H(1,1)-N$ & 0.457 & 0.723 & 0.712 & 0.161 & 0.471 & 0.470 & 0.468 & 0.824 & 0.752 & 0.698 & 0.756 & 0.895 & 0.957 & 0.339 & 0.322 \\
\hline$A R-E G A R C H(1,1)-t$ & 0.066 & 0.825 & 0.180 & 0.006 & 0.097 & 0.097 & 0.097 & 0.913 & 0.876 & 0.848 & 0.253 & 0.431 & 0.599 & 0.057 & 0.392 \\
\hline Monte Carlo Simulation & 0.066 & 0.825 & 0.180 & 0.006 & 0.097 & 0.097 & 0.097 & 0.913 & 0.876 & 0.848 & 0.253 & 0.431 & 0.599 & 0.057 & 0.392 \\
\hline Combination Mean & 0.066 & 0.825 & 0.180 & 0.006 & 0.097 & 0.097 & 0.097 & 0.913 & 0.876 & 0.848 & 0.253 & 0.431 & 0.599 & 0.057 & 0.392 \\
\hline Combination Median & 0.066 & 0.825 & 0.180 & 0.006 & 0.097 & 0.097 & 0.097 & 0.913 & 0.876 & 0.848 & 0.253 & 0.431 & 0.599 & 0.057 & 0.392 \\
\hline Combination Trim1 & 0.025 & 0.860 & 0.079 & 0.231 & 0.049 & 0.050 & 0.050 & 0.938 & 0.911 & 0.891 & 0.144 & 0.275 & 0.423 & 0.017 & 0.313 \\
\hline Combination Trim2 & 0.066 & 0.825 & 0.180 & 0.006 & 0.097 & 0.097 & 0.097 & 0.913 & 0.876 & 0.848 & 0.253 & 0.431 & 0.599 & 0.057 & 0.392 \\
\hline
\end{tabular}

Notes: See Table 2. 
Table 7. Performance Evaluation: All methods

\begin{tabular}{|c|c|c|c|c|c|c|c|c|c|c|c|c|c|c|c|}
\hline \multirow[t]{2}{*}{ Panel A: 5\% } & \multicolumn{3}{|c|}{$\overline{\mathrm{BDI}}$} & \multicolumn{3}{|c|}{$\overline{\mathrm{BPI}}$} & \multicolumn{3}{|c|}{ BCI } & \multicolumn{3}{|c|}{$\overline{\text { BDTI }}$} & \multicolumn{3}{|c|}{ BCTI } \\
\hline & 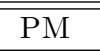 & Ratio & & $\overline{\overline{\mathrm{PM}}}$ & Ratio & & $\overline{\mathrm{PM}}$ & "Ratio & & $\overline{\overline{\mathrm{PM}}}$ & Ratio & & $\overline{\overline{\mathrm{PM}}}$ & Ratio & \\
\hline Filtered Historical Simulation & 0.150 & 3.614 & NR & 0.135 & 3.158 & NR & 0.276 & 4.195 & NR & 0.145 & 4.753 & NR & 0.106 & 5.682 & $\mathrm{R}$ \\
\hline Historical Simulation-250 & 0.313 & 7.538 & $\mathrm{R}$ & 0.301 & 7.072 & $\mathrm{R}$ & 0.326 & 4.956 & NR & 0.216 & 7.094 & $\mathrm{R}$ & 0.106 & 5.674 & $\mathrm{R}$ \\
\hline Historical Simulation-500 & 0.426 & 10.271 & $\mathrm{R}$ & 0.473 & 11.101 & $\mathrm{R}$ & 0.440 & 6.685 & $\mathrm{R}$ & 0.233 & 7.654 & $\mathrm{R}$ & 0.141 & 7.548 & $\mathrm{R}$ \\
\hline Historical SimulationAll & 0.592 & 14.265 & $\mathrm{R}$ & 0.643 & 15.091 & $\mathrm{R}$ & 0.936 & 14.231 & $\mathrm{R}$ & 0.240 & 7.866 & $\mathrm{R}$ & 0.094 & 5.058 & $\mathrm{R}$ \\
\hline Riskmetrics & 0.200 & 4.822 & NR & 0.212 & 4.967 & NR & 0.287 & 4.356 & NR & 0.132 & 4.325 & NR & 0.106 & 5.665 & NR \\
\hline Variance-Covariance & 0.489 & 11.773 & $\mathrm{R}$ & 0.592 & 13.889 & $\mathrm{R}$ & 0.692 & 10.513 & $\mathrm{R}$ & 0.221 & 7.243 & $\mathrm{R}$ & 0.138 & 7.386 & $\mathrm{R}$ \\
\hline Extreme Value Theory & 0.208 & 5.011 & NR & 0.181 & 4.238 & NR & 0.373 & 5.663 & NR & 0.289 & 9.469 & $\mathrm{R}$ & 0.166 & 8.910 & $\mathrm{R}$ \\
\hline AR-ARCH(1)-N & 0.210 & 5.052 & NR & 0.203 & 4.769 & NR & 0.404 & 6.141 & $\mathrm{R}$ & 0.141 & 4.616 & NR & 0.090 & 4.823 & NR \\
\hline $\operatorname{AR}-\operatorname{ARCH}(1)-\mathrm{t}$ & 0.234 & 5.628 & $\mathrm{R}$ & 0.239 & 5.611 & $\mathrm{R}$ & 0.472 & 7.176 & $\mathrm{R}$ & 0.141 & 4.626 & NR & 0.097 & 5.210 & NR \\
\hline AR-GARCH $(1,1)-\mathrm{N}$ & 0.137 & 3.291 & NR & 0.124 & 2.920 & NR & 0.260 & 3.945 & NR & 0.138 & 4.523 & NR & 0.098 & 5.273 & NR \\
\hline $\operatorname{AR}-\operatorname{GARCH}(1,1)-\mathrm{t}$ & 0.139 & 3.341 & NR & 0.133 & 3.117 & NR & 0.270 & 4.104 & NR & 0.142 & 4.647 & NR & 0.099 & 5.286 & NR \\
\hline AR-EGARCH $(1,1)-\mathrm{N}$ & 0.135 & 3.255 & NR & 0.130 & 3.063 & NR & 0.248 & 3.770 & NR & 0.204 & 6.676 & $\mathrm{R}$ & 0.089 & 4.777 & NR \\
\hline $\operatorname{AR}-\operatorname{EGARCH}(1,1)$-t & 0.147 & 3.540 & NR & 0.125 & 2.923 & NR & 0.274 & 4.162 & NR & 0.144 & 4.731 & NR & 0.109 & 5.826 & NR \\
\hline Monte Carlo Simulation & 0.143 & 3.447 & NR & 0.132 & 3.110 & NR & 0.277 & 4.209 & NR & 0.137 & 4.507 & NR & 0.101 & 5.399 & NR \\
\hline Combination Mean & 0.167 & 4.022 & NR & 0.180 & 4.215 & NR & 0.267 & 4.060 & NR & 0.130 & 4.278 & NR & 0.081 & 4.331 & NR \\
\hline Combination Median & 0.142 & 3.419 & NR & 0.129 & 3.032 & NR & 0.248 & 3.769 & NR & 0.132 & 4.339 & NR & 0.079 & 4.250 & NR \\
\hline Combination Trim 1 & 0.162 & 3.896 & NR & 0.169 & 3.971 & NR & 0.265 & 4.030 & NR & 0.132 & 4.331 & NR & 0.084 & 4.479 & NR \\
\hline Combination Trim2 & 0.158 & 3.815 & NR & 0.160 & 3.753 & NR & 0.266 & 4.036 & NR & 0.132 & 4.323 & NR & 0.082 & 4.422 & NR \\
\hline \multirow[t]{2}{*}{ Panel B: 1\% } & & BDI & & & BPI & & & BCI & & &  & & & 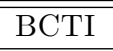 & \\
\hline & $\mathrm{PM}$ & Ratio & & $\mathrm{PM}$ & Ratio & & $\mathrm{PM}$ & Ratio & & $\mathrm{PM}$ & Ratio & & $\mathrm{PM}$ & Ratio & \\
\hline Filtered Histor & 0.023 & 2.699 & NR & 0.018 & 2.014 & NR & 0.036 & 2.812 & NR & 0.021 & 3.212 & NR & 0.021 & 4.273 & NR \\
\hline Historical Simulation-250 & 0.071 & 8.318 & $\mathrm{R}$ & 0.048 & 5.540 & NR & 0.076 & 5.899 & NR & 0.020 & 3.059 & NR & 0.072 & 14.673 & $\mathrm{R}$ \\
\hline Historical Simulation-500 & 0.053 & 6.234 & NR & 0.068 & 7.770 & $\mathrm{R}$ & 0.099 & 7.690 & $\mathrm{R}$ & 0.080 & 12.315 & $\mathrm{R}$ & 0.064 & 13.162 & $\mathrm{R}$ \\
\hline Historical SimulationAll & 0.193 & 22.680 & $\mathrm{R}$ & 0.153 & 17.646 & $\mathrm{R}$ & 0.086 & 6.697 & $\mathrm{R}$ & 0.080 & 12.377 & $\mathrm{R}$ & 0.058 & 11.901 & $\mathrm{R}$ \\
\hline Riskmetrics & 0.047 & 5.525 & NR & 0.036 & 4.125 & NR & 0.063 & 4.912 & NR & 0.027 & 4.176 & NR & 0.021 & 4.313 & NR \\
\hline Variance-Covariance & 0.160 & 18.772 & $\mathrm{R}$ & 0.251 & 28.834 & $\mathrm{R}$ & 0.333 & 26.003 & $\mathrm{R}$ & 0.089 & 13.683 & $\mathrm{R}$ & 0.060 & 12.287 & $\mathrm{R}$ \\
\hline Extreme Value Theory & 0.022 & 2.549 & NR & 0.018 & 2.030 & NR & 0.035 & 2.747 & NR & 0.019 & 2.844 & NR & 0.023 & 4.737 & NR \\
\hline AR-ARCH(1)-N & 0.057 & 6.668 & $\mathrm{R}$ & 0.050 & 5.767 & $\mathrm{R}$ & 0.158 & 12.347 & $\mathrm{R}$ & 0.026 & 3.969 & NR & 0.017 & 3.541 & NR \\
\hline $\operatorname{AR}-\operatorname{ARCH}(1)-\mathrm{t}$ & 0.032 & 3.776 & NR & 0.031 & 3.573 & NR & 0.056 & 4.393 & NR & 0.023 & 3.469 & NR & 0.014 & 2.778 & NR \\
\hline $\operatorname{AR}-\operatorname{GARCH}(1,1)-\mathrm{N}$ & 0.027 & 3.177 & NR & 0.023 & 2.639 & NR & 0.047 & 3.624 & NR & 0.027 & 4.194 & NR & 0.022 & 4.412 & NR \\
\hline AR-GARCH $(1,1)-\mathrm{t}$ & 0.022 & 2.634 & NR & 0.017 & 1.952 & NR & 0.035 & 2.710 & NR & 0.021 & 3.162 & NR & 0.014 & 2.844 & NR \\
\hline $\operatorname{AR-EGARCH}(1,1)-\mathrm{N}$ & 0.027 & 3.120 & NR & 0.022 & 2.568 & NR & 0.047 & 3.665 & NR & 0.093 & 14.399 & $\mathrm{R}$ & 0.020 & 4.011 & NR \\
\hline $\operatorname{AR-EGARCH}(1,1)$-t & 0.021 & 2.417 & NR & 0.017 & 1.908 & NR & 0.034 & 2.628 & NR & 0.019 & 2.981 & NR & 0.015 & 3.149 & NR \\
\hline Monte Carlo Simulation & 0.022 & 2.628 & NR & 0.017 & 1.936 & NR & 0.035 & 2.749 & NR & 0.021 & 3.158 & NR & 0.014 & 2.831 & NR \\
\hline Combination Mean & 0.018 & 2.079 & NR & 0.037 & 4.250 & NR & 0.037 & 2.847 & NR & 0.021 & 3.287 & NR & 0.014 & 2.875 & NR \\
\hline Combination Median & 0.021 & 2.478 & NR & 0.018 & 2.064 & NR & 0.037 & 2.876 & NR & 0.022 & 3.426 & NR & 0.014 & 2.845 & NR \\
\hline Combination Trim1 & 0.017 & 2.007 & NR & 0.022 & 2.573 & NR & 0.033 & 2.576 & NR & 0.020 & 3.053 & NR & 0.013 & 2.648 & NR \\
\hline Combination Trim2 & 0.019 & 2.239 & NR & 0.024 & 2.810 & NR & 0.036 & 2.825 & NR & 0.021 & 3.234 & NR & 0.013 & 2.720 & NR \\
\hline
\end{tabular}

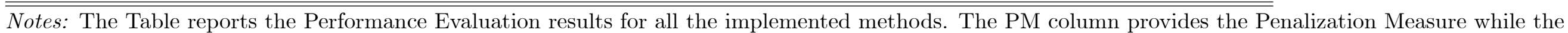
Ratio column provides the corresponding ratio. $R(N R)$ suggests rejection (non-rejection) with respect to the performance equality test. 
Table 8. Performance Evaluation: Second Stage

\begin{tabular}{|c|c|c|c|c|c|c|c|c|c|c|c|c|c|c|c|}
\hline \multirow[t]{2}{*}{ Panel A: 5\% } & \multicolumn{3}{|c|}{$\overline{\mathrm{BDI}}$} & \multicolumn{3}{|c|}{ BPI } & \multicolumn{3}{|c|}{$\overline{\mathrm{BCI}}$} & \multicolumn{3}{|c|}{$\overline{\text { BDTI }}$} & \multicolumn{3}{|c|}{ BCTI } \\
\hline & 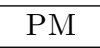 & Ratio & & $\overline{P M}$ & Ratio & & $\overline{\mathrm{PM}}$ & Ratio & & $\overline{\mathrm{PM}}$ & Ratio & & $\overline{\mathrm{PM}}$ & Ratio & \\
\hline Filtered Historical Simulation & 0.150 & 6.437 & NR & 0.135 & 5.976 & NR & 0.276 & 6.162 & NR & 0.145 & 7.834 & $\mathrm{R}$ & 0.106 & 7.461 & $\mathrm{R}$ \\
\hline Historical Simulation-250 & - & - & - & - & - & - & 0.326 & 7.280 & NR & - & - & - & 0.106 & 7.451 & $\mathrm{R}$ \\
\hline Historical Simulation-500 & - & - & - & - & - & - & 0.440 & 9.819 & $\mathrm{R}$ & - & - & - & - & - & - \\
\hline Historical SimulationAll & - & - & - & - & - & - & 0.000 & - & - & - & - & - & 0.094 & 6.642 & $\mathrm{R}$ \\
\hline Riskmetrics & 0.200 & 8.587 & $\mathrm{R}$ & 0.212 & 9.400 & $\mathrm{R}$ & 0.287 & 6.398 & $\mathrm{R}$ & 0.132 & 7.128 & NR & 0.106 & 7.439 & $\mathrm{R}$ \\
\hline Variance-Covariance & - & - & - & - & - & - & 0.000 & - & - & - & - & - & - & - & - \\
\hline Extreme Value Theory & 0.208 & 8.923 & $\mathrm{R}$ & 0.181 & 8.019 & $\mathrm{R}$ & 0.373 & 8.318 & $\mathrm{R}$ & - & - & - & - & - & - \\
\hline AR-ARCH(1)-N & .210 & 8.997 & $\mathrm{R}$ & .203 & 9.025 & $\mathrm{R}$ & 0.404 & 9.020 & $\mathrm{R}$ & 0.141 & 7.607 & NR & 0.090 & 6.333 & NR \\
\hline $\mathrm{AR}-\mathrm{ARCH}(1)-\mathrm{t}$ & 234 & 10.023 & $\mathrm{R}$ & 239 & 10.618 & $\mathrm{R}$ & 0.000 & - & - & 0.141 & 7.624 & $\mathrm{R}$ & 0.097 & 6.841 & $\mathrm{R}$ \\
\hline AR-C & 137 & 5.861 & NR & 24 & 5.525 & NR & 0.260 & 5.795 & NR & 0.138 & 7.455 & NR & 0.098 & 6.924 & NR \\
\hline $\mathrm{RCH}$ & 139 & 5.950 & NR & 133 & 5.898 & NR & 0.270 & 6.028 & NR & 0.142 & 7.659 & $\mathrm{R}$ & 0.099 & 6.941 & $\mathrm{R}$ \\
\hline AR-EGARCH(1,1)-N & 135 & 5.797 & NR & .130 & 5.795 & NR & 0.248 & 5.537 & NR & 0.204 & 11.002 & $\mathrm{R}$ & 0.089 & 6.272 & NR \\
\hline AR-EGARCH $(1,1)$-t & 147 & 6.304 & NR & .125 & 5.530 & NR & 0.274 & 6.113 & NR & 0.144 & 7.798 & $\mathrm{R}$ & 0.109 & 7.650 & $\mathrm{R}$ \\
\hline Monte Carlo Simulatio & 143 & 6.138 & NR & 32 & 5.884 & $\mathrm{NR}$ & 0.277 & 6.182 & NR & 0.137 & 7.428 & NR & 0.101 & 7.090 & $\mathrm{R}$ \\
\hline Com & 167 & 7.162 & NR & & 7.976 & $\mathrm{R}$ & 0.267 & 5.964 & NR & 0.130 & 7.050 & NR & 0.081 & 5.688 & NR \\
\hline Com & 142 & 6.089 & NR & 129 & 5.737 & NR & 0.248 & 5.536 & NR & 0.132 & 7.152 & $\mathrm{NR}$ & 0.079 & 5.581 & NR \\
\hline Com & 162 & 6.938 & NR & 169 & 7.514 & $\mathrm{R}$ & 0.265 & 5.920 & NR & 0.132 & 7.138 & NR & 0.084 & 5.882 & NR \\
\hline Combination Trim2 & 158 & 6.795 & NR & 0.160 & 7.102 & NR & 0.266 & 5.929 & NR & 0.132 & 7.124 & $\mathrm{NR}$ & 0.082 & 5.806 & NR \\
\hline \multirow[t]{2}{*}{ Panel B: 1\% } & & BDI & & & BPI & & & BCI & & & BDTI & & & BCTI & \\
\hline & $\overline{P \mathrm{PM}}$ & Ratio & & PM & Ratio & & PM & Ratio & & PM & Ratio & & PM & Ratio & \\
\hline Filte & 0.023 & 6.195 & $\mathrm{NR}$ & 0.018 & 5.037 & NR & 0.036 & 5.211 & NR & 0.021 & 6.802 & NR & 0.021 & 8.906 & $\mathrm{R}$ \\
\hline Histo & - & - & - & 0.048 & 13.85 & $\mathrm{R}$ & 0.076 & 10.933 & $\mathrm{R}$ & 0.020 & 6.478 & $\mathrm{R}$ & - & - & - \\
\hline Histo & 0.053 & 14.310 & $\mathrm{R}$ & - & - & - & - & - & - & - & - & - & - & - & - \\
\hline Historical Simu & - & - & - & - & - & - & 0.086 & 12.412 & $\mathrm{R}$ & - & - & - & - & - & - \\
\hline Riskmetrics & 0.047 & 2.683 & $\mathrm{R}$ & 0.036 & 10.31 & $\mathrm{R}$ & 0.063 & 9.103 & $\mathrm{R}$ & 0.027 & 8.842 & $\mathrm{R}$ & 0.021 & 8.989 & $\mathrm{R}$ \\
\hline Varia & - & - & - & - & - & - & - & - & - & - & - & - & - & - & - \\
\hline Extreme Value Theory & 0.022 & 5.851 & NR & 0.018 & 5.076 & NR & 0.035 & 5.090 & NR & 0.019 & 6.022 & NR & 0.023 & 9.875 & $\mathrm{R}$ \\
\hline AR-ARCH(1)-N & - & - & - & - & - & - & - & - & - & 0.026 & 8.404 & $\mathrm{R}$ & 0.017 & 7.380 & $\mathrm{R}$ \\
\hline $\mathrm{AR}-\mathrm{ARCH}(1)-\mathrm{t}$ & .032 & 8.668 & $\mathrm{R}$ & 0.031 & 8.936 & $\mathrm{R}$ & 0.056 & 8.141 & $\mathrm{R}$ & 0.023 & 7.346 & NR & 0.014 & 5.791 & NR \\
\hline $\operatorname{AR}-\operatorname{GARCH}(1,1$ & 0.027 & 7.294 & $\mathrm{R}$ & 0.023 & 6.601 & NR & 0.047 & 6.716 & $\mathrm{R}$ & 0.027 & 8.880 & $\mathrm{R}$ & 0.022 & 9.196 & $\mathrm{R}$ \\
\hline AR-GARCH & 0.022 & 6.047 & NR & 0.017 & 4.882 & NR & 0.035 & 5.022 & NR & 0.021 & 6.696 & NR & 0.014 & 5.927 & NR \\
\hline AR-EGARCH $(1,1)-\mathrm{N}$ & 0.027 & 7.162 & $\mathrm{R}$ & 0.022 & 6.423 & NR & 0.047 & 6.791 & $\mathrm{R}$ & - & - & - & 0.020 & 8.360 & $\mathrm{R}$ \\
\hline AR-EGARCH(1,1)-t & 0.021 & 5.548 & NR & 0.017 & 4.773 & NR & 0.034 & 4.871 & NR & 0.019 & 6.312 & NR & 0.015 & 6.563 & NR \\
\hline Monte Carlo Simulation & 0.022 & 6.032 & NR & 0.017 & 4.842 & NR & 0.035 & 5.095 & NR & 0.021 & 6.688 & NR & 0.014 & 5.901 & NR \\
\hline Combination Mean & 0.018 & 4.772 & NR & 0.037 & 10.631 & $\mathrm{R}$ & 0.037 & 5.277 & NR & 0.021 & 6.961 & NR & 0.014 & 5.993 & NR \\
\hline Combination Median & 0.021 & 5.688 & NR & 0.018 & 5.162 & NR & 0.037 & 5.330 & NR & 0.022 & 7.255 & $\mathrm{R}$ & 0.014 & 5.931 & NR \\
\hline Combination Trim1 & 0.017 & 4.608 & NR & 0.022 & 6.435 & $\mathrm{NR}$ & 0.033 & 4.773 & NR & 0.020 & 6.465 & $\mathrm{NR}$ & 0.013 & 5.519 & NR \\
\hline Combination Trim2 & 0.019 & 5.141 & NR & 0.024 & 7.028 & $\mathrm{R}$ & 0.036 & 5.235 & NR & 0.021 & 6.849 & NR & 0.013 & 5.670 & NR \\
\hline
\end{tabular}

Notes: The Table reports the second stage Performance Evaluation results. For each index the worst performing methods are excluded and the analysis is repeated with the remaining methods. The PM column provides the Penalization Measure while the Ratio column provides the corresponding ratio. $R(N R)$ suggests rejection (non-rejection) with respect to the performance equality test. 
Table 9. Ranking and Performance Equality Test

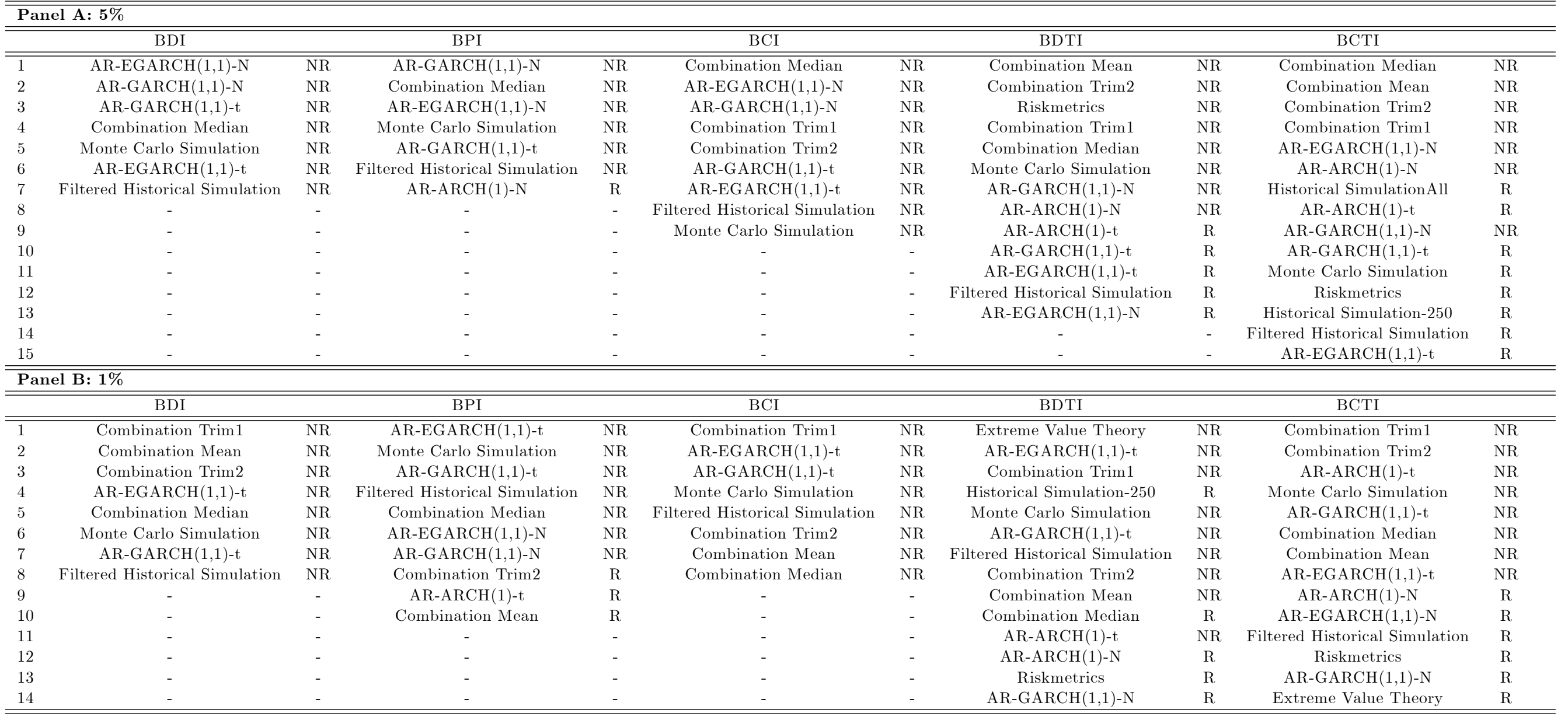

Notes: The Table reports the ranking of the statistically accepted methods according to the PM measure presented in Table 8 . $R(N R)$ suggests rejection (non-rejection) with respect to the performance equality test. 


\section{Appendix A: Individual Route Indices}

Table A1. Descriptive Statistics

\begin{tabular}{|c|c|c|c|c|c|c|}
\hline & 4TC AVG CAPE & 4TC AVG PANAM & $\overline{\text { TD3 }}$ & $\overline{\text { TD5 }}$ & $\overline{\text { TD7 }}$ & TD9 \\
\hline Mean & -0.0353 & -0.0051 & 0.0069 & 0.0093 & 0.0043 & 0.0001 \\
\hline Median & 0.0100 & 0.0483 & -0.2432 & -0.1363 & -0.1528 & -0.0881 \\
\hline Maximum & 31.497 & 13.112 & 39.961 & 55.587 & 42.700 & 47.923 \\
\hline Minimum & -34.203 & -21.663 & -50.199 & -40.180 & -49.959 & -51.748 \\
\hline Std. Dev. & 3.679 & 2.414 & 5.149 & 4.776 & 5.202 & 6.150 \\
\hline Skewness & 0.326 & -0.380 & 0.155 & 0.694 & 0.797 & 0.713 \\
\hline Kurtosis & 13.675 & 10.714 & 15.929 & 15.968 & 17.776 & 16.226 \\
\hline Jarque-Bera & 14731.09 & 7737.52 & 21539.86 & 21905.46 & 28445.34 & 22790.52 \\
\hline Probability & 0.00 & 0.00 & 0.00 & 0.00 & 0.00 & 0.00 \\
\hline $\mathrm{Q}(1)$ & 1734.30 & 2229.20 & 807.30 & 525.07 & 767.06 & 417.79 \\
\hline Probability & 0.00 & 0.00 & 0.00 & 0.00 & 0.00 & 0.00 \\
\hline $\mathrm{Q}(10)$ & 2992.50 & 3997.40 & 1228.10 & 726.69 & 1395.50 & 608.74 \\
\hline Probability & 0.00 & 0.00 & 0.00 & 0.00 & 0.00 & 0.00 \\
\hline $\mathrm{Q}(1) \mathrm{sq}$ & 630.94 & 1621.70 & 96.86 & 25.41 & 46.26 & 80.51 \\
\hline Probability & 0.00 & 0.00 & 0.00 & 0.00 & 0.00 & 0.00 \\
\hline $\mathrm{Q}(10) \mathrm{sq}$ & 1607.00 & 4013.20 & 208.61 & 36.39 & 103.27 & 104.48 \\
\hline Probability & 0.00 & 0.00 & 0.00 & 0.00 & 0.00 & 0.00 \\
\hline Observations & 3091 & 3091 & 3091 & 3091 & 3091 & 3091 \\
\hline
\end{tabular}

Notes: Descriptive statistics are calculated for the whole sample of logarithmic returns covering the period 1/11/1999 -12/03/2012.

The Jarque-Bera statistic for the normality test is $\chi^{2}(2)$ distributed.

$\mathrm{Q}(\mathrm{i})$ and $\mathrm{Q}(\mathrm{i})$ sq are the Ljung-Box Q statistics for the returns and squared returns at the $i$ lag. 
Table A2. Backtesting results 4 Time Charter Average Capesize

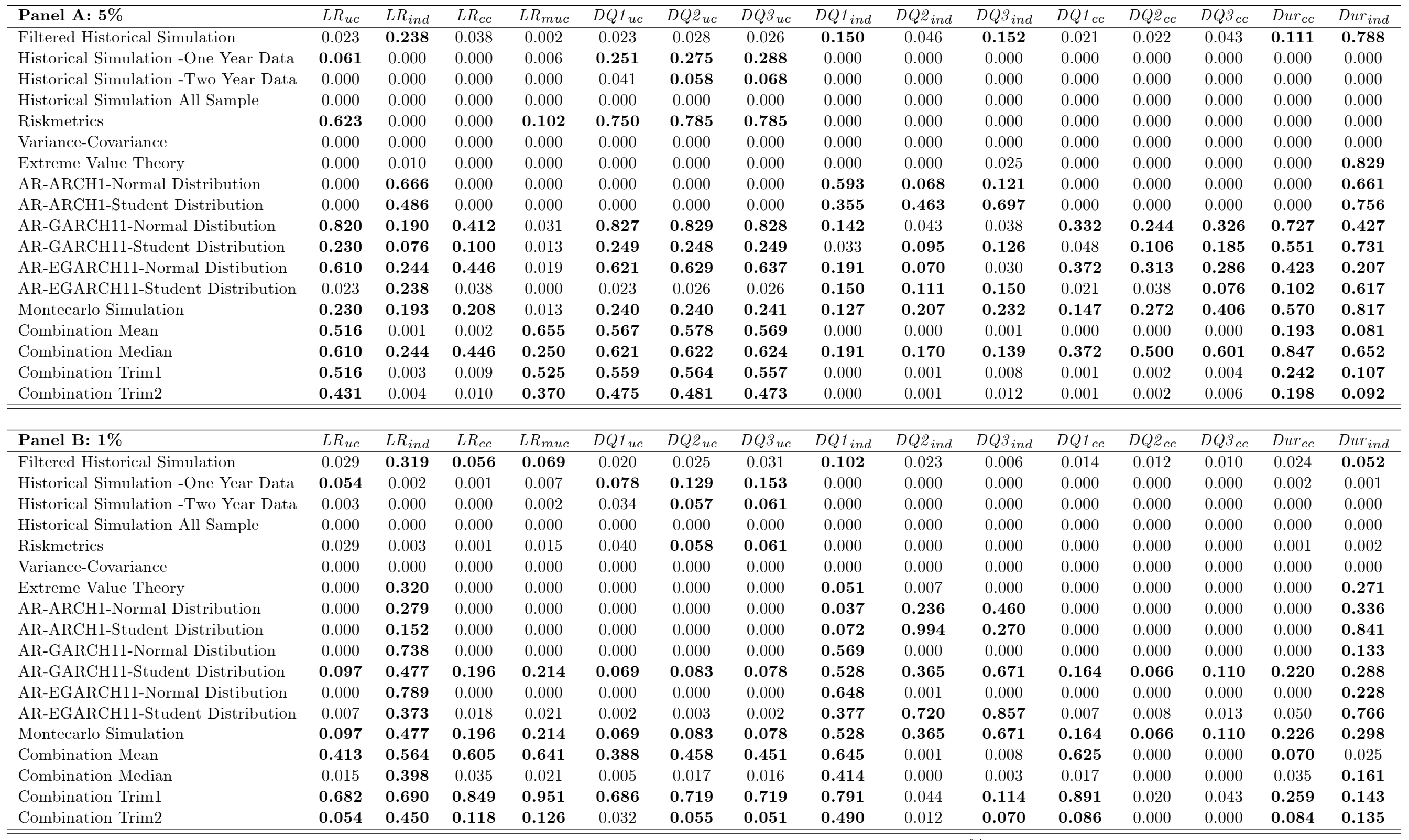

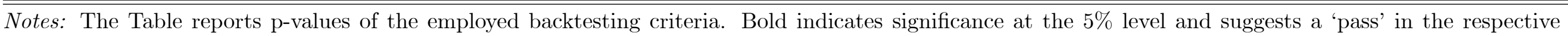
criterion. 
Table A3. Backtesting results 4 Time Charter Average Panamax

\begin{tabular}{|c|c|c|c|c|c|c|c|c|c|c|c|c|c|c|c|}
\hline Panel A: 5\% & $L R_{u c}$ & $L R_{\text {ind }}$ & $L R_{c c}$ & $L R_{m u c}$ & $D Q 1 u c$ & $\overline{D Q 2 u c}$ & DQ3uc & $D Q 1_{\text {ind }}$ & $D Q 2_{\text {ind }}$ & $D Q 3_{\text {ind }}$ & $D Q 1 c c$ & $D Q 2 c c$ & $D Q 3 c c$ & Dur $_{c c}$ & Dur $_{\text {ind }}$ \\
\hline Filtered Historical Simulation & 0.820 & 0.190 & 0.412 & 0.868 & 0.827 & 0.829 & 0.824 & 0.142 & 0.043 & 0.087 & 0.332 & 0.244 & 0.384 & 0.701 & 0.401 \\
\hline Historical Simulation -One Year Data & 0.061 & 0.000 & 0.000 & 0.000 & 0.566 & 0.535 & 0.603 & 0.000 & 0.000 & 0.000 & 0.000 & 0.000 & 0.000 & 0.000 & 0.000 \\
\hline Historical Simulation - Two Year Data & 0.002 & 0.000 & 0.000 & 0.000 & 0.226 & 0.249 & 0.257 & 0.000 & 0.000 & 0.000 & 0.000 & 0.000 & 0.000 & 0.000 & 0.000 \\
\hline Historical Simulation All Sample & 0.000 & 0.000 & 0.000 & 0.000 & 0.004 & 0.005 & 0.007 & 0.000 & 0.000 & 0.000 & 0.000 & 0.000 & 0.000 & 0.000 & 0.000 \\
\hline Riskmetrics & 0.712 & 0.000 & 0.000 & 0.036 & 0.839 & 0.838 & 0.830 & 0.000 & 0.000 & 0.000 & 0.000 & 0.000 & 0.000 & 0.000 & 0.000 \\
\hline Variance-Covariance & 0.000 & 0.000 & 0.000 & 0.000 & 0.051 & 0.041 & 0.038 & 0.000 & 0.000 & 0.000 & 0.000 & 0.000 & 0.000 & 0.000 & 0.000 \\
\hline Extreme Value Theory & 0.000 & 0.002 & 0.000 & 0.000 & 0.000 & 0.000 & 0.000 & 0.000 & 0.000 & 0.000 & 0.000 & 0.000 & 0.000 & 0.000 & 0.378 \\
\hline AR-ARCH1-Normal Distribution & 0.729 & 0.295 & 0.544 & 0.378 & 0.741 & 0.749 & 0.767 & 0.264 & 0.278 & 0.059 & 0.505 & 0.677 & 0.330 & 0.174 & 0.071 \\
\hline AR-GARCH11-Normal Distibution & 0.278 & 0.431 & 0.407 & 0.574 & 0.303 & 0.307 & 0.323 & 0.430 & 0.544 & 0.345 & 0.418 & 0.630 & 0.675 & 0.458 & 0.744 \\
\hline AR-GARCH11-Student Distribution & 0.954 & 0.366 & 0.664 & 0.853 & 0.956 & 0.963 & 0.969 & 0.333 & 0.071 & 0.105 & 0.624 & 0.322 & 0.474 & 0.813 & 0.541 \\
\hline AR-EGARCH11-Normal Distibution & 0.729 & 0.002 & 0.007 & 0.970 & 0.763 & 0.767 & 0.775 & 0.000 & 0.005 & 0.010 & 0.001 & 0.002 & 0.005 & 0.443 & 0.237 \\
\hline AR-EGARCH11-Student Distribution & 0.841 & 0.002 & 0.009 & 0.813 & 0.860 & 0.864 & 0.871 & 0.000 & 0.006 & 0.014 & 0.001 & 0.003 & 0.008 & 0.326 & 0.145 \\
\hline Montecarlo Simulation & 0.954 & 0.144 & 0.343 & 0.474 & 0.957 & 0.963 & 0.969 & 0.104 & 0.074 & 0.109 & 0.265 & 0.330 & 0.483 & 0.763 & 0.478 \\
\hline Combination Mean & 0.008 & 0.001 & 0.000 & 0.017 & 0.035 & 0.058 & 0.098 & 0.000 & 0.000 & 0.000 & 0.000 & 0.000 & 0.000 & 0.000 & 0.000 \\
\hline Combination Median & 0.045 & 0.012 & 0.006 & 0.147 & 0.085 & 0.088 & 0.104 & 0.006 & 0.032 & 0.015 & 0.004 & 0.011 & 0.015 & 0.046 & 0.192 \\
\hline Combination Trim1 & 0.013 & 0.001 & 0.000 & 0.022 & 0.045 & 0.071 & 0.113 & 0.000 & 0.000 & 0.000 & 0.000 & 0.000 & 0.000 & 0.000 & 0.000 \\
\hline Combination Trim2 & 0.013 & 0.001 & 0.000 & 0.010 & 0.045 & 0.062 & 0.093 & 0.000 & 0.000 & 0.000 & 0.000 & 0.000 & 0.000 & 0.000 & 0.001 \\
\hline Panel B: 1\% & $L R_{u c}$ & $L R_{\text {ind }}$ & $L R_{c c}$ & $L R_{m u c}$ & $D Q 1 u c$ & DQ2uc & $D Q 3 u c$ & $D Q 1_{\text {ind }}$ & $D Q^{2}$ ind & $D Q 3_{i n d}$ & $D Q 1 c c$ & $D Q^{2} c c$ & $D Q 3 c c$ & Dur $_{c c}$ & Dur $_{\text {ind }}$ \\
\hline Filtered Historical Simulation & 0.269 & 0.534 & 0.447 & 0.485 & 0.237 & 0.230 & 0.257 & 0.606 & 0.462 & 0.499 & 0.444 & 0.592 & 0.156 & 0.559 & 0.479 \\
\hline Historical Simulation -One Year Data & 0.029 & 0.000 & 0.000 & 0.015 & 0.172 & 0.253 & 0.201 & 0.000 & 0.000 & 0.000 & 0.000 & 0.000 & 0.000 & 0.000 & 0.000 \\
\hline Historical Simulation - Two Year Data & 0.054 & 0.000 & 0.000 & 0.007 & 0.314 & 0.282 & 0.333 & 0.000 & 0.000 & 0.000 & 0.000 & 0.000 & 0.000 & 0.000 & 0.000 \\
\hline Historical Simulation All Sample & 0.001 & 0.000 & 0.000 & 0.000 & 0.216 & 0.173 & 0.206 & 0.000 & 0.000 & 0.000 & 0.000 & 0.000 & 0.000 & 0.000 & 0.000 \\
\hline Riskmetrics & 0.015 & 0.000 & 0.000 & 0.036 & 0.108 & 0.119 & 0.143 & 0.000 & 0.000 & 0.000 & 0.000 & 0.000 & 0.000 & 0.000 & 0.000 \\
\hline Variance-Covariance & 0.000 & 0.000 & 0.000 & 0.000 & 0.000 & 0.001 & 0.002 & 0.000 & 0.000 & 0.000 & 0.000 & 0.000 & 0.000 & 0.000 & 0.000 \\
\hline Extreme Value Theory & 0.007 & 0.373 & 0.018 & 0.021 & 0.002 & 0.003 & 0.004 & 0.377 & 0.720 & 0.266 & 0.007 & 0.008 & 0.007 & 0.052 & 0.963 \\
\hline AR-ARCH1-Normal Distribution & 0.003 & 0.349 & 0.008 & 0.002 & 0.001 & 0.001 & 0.001 & 0.342 & 0.823 & 0.350 & 0.002 & 0.003 & 0.004 & 0.005 & 0.072 \\
\hline AR-ARCH1-Student Distribution & 0.166 & 0.505 & 0.307 & 0.026 & 0.134 & 0.127 & 0.121 & 0.567 & 0.414 & 0.313 & 0.284 & 0.413 & 0.520 & 0.013 & 0.006 \\
\hline AR-GARCH11-Normal Distibution & 0.054 & 0.450 & 0.118 & 0.125 & 0.032 & 0.040 & 0.050 & 0.490 & 0.443 & 0.097 & 0.086 & 0.046 & 0.023 & 0.256 & 0.929 \\
\hline AR-GARCH11-Student Distribution & 0.823 & 0.626 & 0.866 & 0.555 & 0.820 & 0.815 & 0.810 & 0.720 & 0.610 & 0.529 & 0.914 & 0.958 & 0.978 & 0.804 & 0.513 \\
\hline AR-EGARCH11-Normal Distibution & 0.097 & 0.477 & 0.196 & 0.130 & 0.069 & 0.083 & 0.099 & 0.528 & 0.365 & 0.064 & 0.164 & 0.066 & 0.026 & 0.194 & 0.240 \\
\hline AR-EGARCH11-Student Distribution & 0.930 & 0.658 & 0.903 & 0.788 & 0.929 & 0.931 & 0.933 & 0.756 & 0.659 & 0.586 & 0.949 & 0.977 & 0.990 & 0.707 & 0.468 \\
\hline Montecarlo Simulation & 0.930 & 0.658 & 0.903 & 0.664 & 0.929 & 0.931 & 0.933 & 0.756 & 0.659 & 0.586 & 0.949 & 0.977 & 0.990 & 0.332 & 0.153 \\
\hline Combination Mean & 0.457 & 0.048 & 0.107 & 0.679 & 0.529 & 0.575 & 0.613 & 0.001 & 0.000 & 0.000 & 0.003 & 0.000 & 0.000 & 0.081 & 0.050 \\
\hline Combination Median & 0.275 & 0.757 & 0.526 & 0.332 & 0.301 & 0.299 & 0.297 & 0.856 & 0.796 & 0.751 & 0.580 & 0.773 & 0.887 & 0.257 & 0.432 \\
\hline
\end{tabular}

Notes: See Table 2. 
Table A4. Backtesting results TD3

\begin{tabular}{|c|c|c|c|c|c|c|c|c|c|c|c|c|c|c|c|}
\hline Panel A: 5\% & $L R_{u c}$ & $L R_{\text {ind }}$ & $L R_{c c}$ & $L R_{m u c}$ & $D Q 1 u c$ & $\overline{D Q 2 u c}$ & DQ3uc & $D Q 1_{\text {ind }}$ & $D Q 2_{\text {ind }}$ & $D Q 3_{\text {ind }}$ & $D Q 1 c c$ & $D Q 2 c c$ & $D Q 3 c c$ & Dur $_{c c}$ & Dur $_{\text {ind }}$ \\
\hline Filtered Historical Simulation & 0.278 & 0.002 & 0.005 & 0.317 & 0.353 & 0.361 & 0.399 & 0.000 & 0.003 & 0.000 & 0.001 & 0.003 & 0.001 & 0.325 & 0.373 \\
\hline Historical Simulation -One Year Data & 0.217 & 0.000 & 0.000 & 0.293 & 0.398 & 0.421 & 0.477 & 0.000 & 0.000 & 0.000 & 0.000 & 0.000 & 0.000 & 0.000 & 0.000 \\
\hline Historical Simulation -Two Year Data & 0.064 & 0.000 & 0.000 & 0.202 & 0.203 & 0.255 & 0.269 & 0.000 & 0.000 & 0.000 & 0.000 & 0.000 & 0.000 & 0.000 & 0.000 \\
\hline Historical Simulation All Sample & 0.045 & 0.000 & 0.000 & 0.158 & 0.191 & 0.206 & 0.235 & 0.000 & 0.000 & 0.000 & 0.000 & 0.000 & 0.000 & 0.000 & 0.000 \\
\hline Riskmetrics & 0.432 & 0.000 & 0.000 & 0.771 & 0.574 & 0.593 & 0.598 & 0.000 & 0.000 & 0.000 & 0.000 & 0.000 & 0.000 & 0.012 & 0.004 \\
\hline Variance-Covariance & 0.000 & 0.000 & 0.000 & 0.000 & 0.003 & 0.007 & 0.012 & 0.000 & 0.000 & 0.000 & 0.000 & 0.000 & 0.000 & 0.000 & 0.001 \\
\hline Extreme Value Theory & 0.000 & 0.053 & 0.000 & 0.000 & 0.000 & 0.000 & 0.000 & 0.007 & 0.000 & 0.000 & 0.000 & 0.000 & 0.000 & 0.000 & 0.249 \\
\hline AR-ARCH1-Normal Distribution & 0.000 & 0.373 & 0.000 & 0.000 & 0.000 & 0.000 & 0.000 & 0.687 & 0.366 & 0.255 & 0.000 & 0.000 & 0.000 & 0.000 & 0.410 \\
\hline AR-GARCH11-Normal Distibution & 0.001 & 0.063 & 0.001 & 0.006 & 0.005 & 0.009 & 0.020 & 0.071 & 0.014 & 0.001 & 0.002 & 0.001 & 0.000 & 0.003 & 0.935 \\
\hline AR-GARCH11-Student Distribution & 0.841 & 0.765 & 0.937 & 0.915 & 0.840 & 0.857 & 0.867 & 0.774 & 0.139 & 0.067 & 0.940 & 0.125 & 0.141 & 0.810 & 0.579 \\
\hline AR-EGARCH11-Normal Distibution & 0.000 & 0.027 & 0.000 & 0.000 & 0.001 & 0.002 & 0.005 & 0.033 & 0.023 & 0.003 & 0.000 & 0.000 & 0.000 & 0.000 & 0.557 \\
\hline AR-EGARCH11-Student Distribution & 0.623 & 0.612 & 0.779 & 0.050 & 0.632 & 0.650 & 0.646 & 0.607 & 0.232 & 0.621 & 0.778 & 0.595 & 0.616 & 0.739 & 0.641 \\
\hline Montecarlo Simulation & 0.350 & 0.982 & 0.646 & 0.645 & 0.360 & 0.399 & 0.400 & 0.983 & 0.131 & 0.098 & 0.657 & 0.150 & 0.214 & 0.496 & 0.596 \\
\hline Combination Mean & 0.020 & 0.001 & 0.000 & 0.029 & 0.058 & 0.068 & 0.000 & 0.000 & 0.001 & 0.000 & 0.000 & 0.000 & 0.000 & 0.030 & 0.288 \\
\hline Combination Median & 0.003 & 0.090 & 0.003 & 0.008 & 0.010 & 0.016 & 0.029 & 0.099 & 0.024 & 0.002 & 0.005 & 0.005 & 0.001 & 0.009 & 0.880 \\
\hline Combination Trim1 & 0.020 & 0.001 & 0.000 & 0.029 & 0.058 & 0.068 & 0.000 & 0.000 & 0.001 & 0.000 & 0.000 & 0.000 & 0.000 & 0.030 & 0.288 \\
\hline Combination Trim2 & 0.020 & 0.001 & 0.000 & 0.029 & 0.058 & 0.068 & 0.000 & 0.000 & 0.001 & 0.000 & 0.000 & 0.000 & 0.000 & 0.030 & 0.288 \\
\hline Panel B: 1\% & $L R_{u c}$ & $L R_{\text {ind }}$ & $L R_{c c}$ & $L R_{m u c}$ & $D Q 1 u c$ & $D Q 2 u c$ & DQ3uc & $D Q 1_{\text {ind }}$ & $D Q 2_{\text {ind }}$ & $D Q 3_{\text {ind }}$ & $D Q 1_{c c}$ & $D Q 2 c c$ & $D Q 3 c c$ & Dur $_{c c}$ & Dur $_{\text {ind }}$ \\
\hline Filtered Historical Simulation & 0.066 & 0.825 & 0.180 & 0.043 & 0.097 & 0.097 & 0.097 & 0.913 & 0.876 & 0.848 & 0.253 & 0.431 & 0.599 & 0.053 & 0.355 \\
\hline Historical Simulation -One Year Data & 0.413 & 0.009 & 0.023 & 0.641 & 0.465 & 0.448 & 0.444 & 0.000 & 0.002 & 0.015 & 0.000 & 0.000 & 0.000 & 0.298 & 0.143 \\
\hline Historical Simulation - Two Year Data & 0.823 & 0.004 & 0.016 & 0.929 & 0.852 & 0.844 & 0.841 & 0.000 & 0.000 & 0.004 & 0.000 & 0.000 & 0.000 & 0.449 & 0.207 \\
\hline Historical Simulation All Sample & 0.066 & 0.015 & 0.010 & 0.152 & 0.185 & 0.167 & 0.171 & 0.000 & 0.006 & 0.018 & 0.000 & 0.000 & 0.000 & 0.061 & 0.447 \\
\hline Riskmetrics & 0.001 & 0.000 & 0.000 & 0.005 & 0.004 & 0.005 & 0.008 & 0.000 & 0.000 & 0.000 & 0.000 & 0.000 & 0.000 & 0.005 & 0.172 \\
\hline Variance-Covariance & 0.682 & 0.002 & 0.006 & 0.951 & 0.753 & 0.741 & 0.744 & 0.000 & 0.000 & 0.001 & 0.000 & 0.000 & 0.000 & 0.141 & 0.067 \\
\hline Extreme Value Theory & 0.413 & 0.564 & 0.605 & 0.452 & 0.388 & 0.380 & 0.410 & 0.645 & 0.511 & 0.407 & 0.625 & 0.761 & 0.158 & 0.743 & 0.572 \\
\hline AR-ARCH1-Normal Distribution & 0.823 & 0.626 & 0.866 & 0.827 & 0.820 & 0.815 & 0.827 & 0.720 & 0.610 & 0.250 & 0.914 & 0.958 & 0.112 & 0.894 & 0.643 \\
\hline AR-ARCH1-Student Distribution & 0.146 & 0.791 & 0.335 & 0.328 & 0.178 & 0.177 & 0.176 & 0.885 & 0.838 & 0.801 & 0.402 & 0.607 & 0.763 & 0.176 & 0.732 \\
\hline AR-GARCH11-Normal Distibution & 0.823 & 0.103 & 0.258 & 0.827 & 0.836 & 0.846 & 0.854 & 0.007 & 0.000 & 0.000 & 0.026 & 0.004 & 0.001 & 0.761 & 0.463 \\
\hline AR-GARCH11-Student Distribution & 0.457 & 0.723 & 0.712 & 0.464 & 0.471 & 0.470 & 0.526 & 0.824 & 0.752 & 0.092 & 0.756 & 0.895 & 0.019 & 0.395 & 0.410 \\
\hline AR-EGARCH11-Normal Distibution & 0.823 & 0.103 & 0.258 & 0.827 & 0.836 & 0.846 & 0.869 & 0.007 & 0.000 & 0.000 & 0.026 & 0.004 & 0.000 & 0.245 & 0.094 \\
\hline AR-EGARCH11-Student Distribution & 0.146 & 0.791 & 0.335 & 0.127 & 0.178 & 0.177 & 0.259 & 0.885 & 0.838 & 0.032 & 0.402 & 0.607 & 0.001 & 0.136 & 0.422 \\
\hline Montecarlo Simulation & 0.275 & 0.757 & 0.526 & 0.075 & 0.301 & 0.299 & 0.372 & 0.856 & 0.796 & 0.058 & 0.580 & 0.773 & 0.006 & 0.313 & 0.634 \\
\hline Combination Mean & 0.025 & 0.860 & 0.079 & 0.010 & 0.049 & 0.050 & 0.050 & 0.938 & 0.911 & 0.891 & 0.144 & 0.275 & 0.423 & 0.023 & 0.555 \\
\hline Combination Median & 0.066 & 0.825 & 0.180 & 0.043 & 0.097 & 0.097 & 0.097 & 0.913 & 0.876 & 0.848 & 0.253 & 0.431 & 0.599 & 0.076 & 0.715 \\
\hline
\end{tabular}

Notes: See Table 2. 
Table A5. Backtesting results TD5

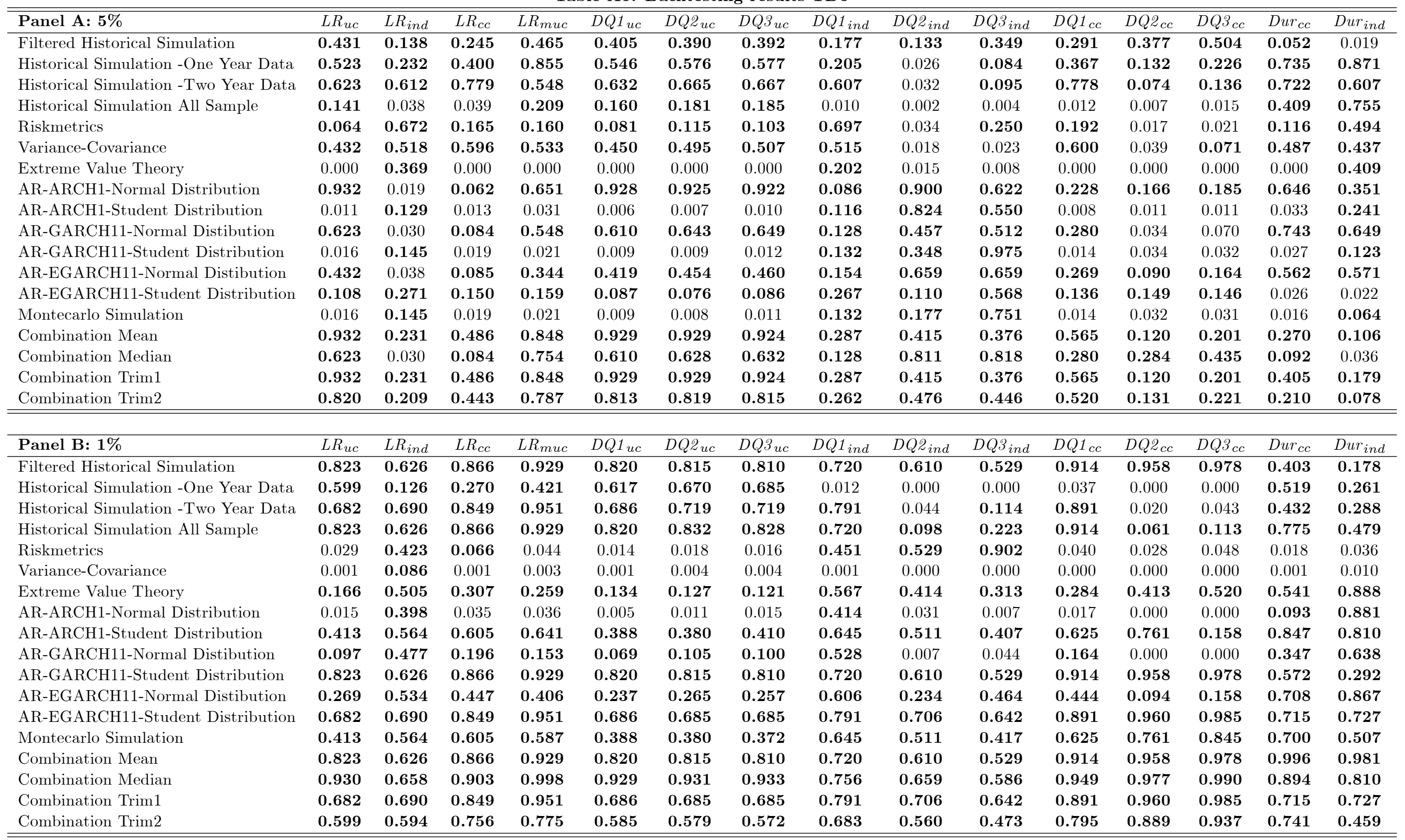

Notes: See Table 2 
Table A6. Backtesting results TD7

\begin{tabular}{|c|c|c|c|c|c|c|c|c|c|c|c|c|c|c|c|}
\hline Panel A: 5\% & 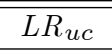 & 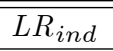 & $\overline{L L R_{c c}}$ & 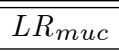 & $D Q 1 u c$ & $\overline{D Q 2 u c}$ & 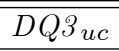 & $D Q 1_{\text {ind }}$ & $D Q 2_{\text {ind }}$ & $D Q 3_{\text {ind }}$ & 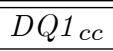 & 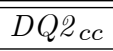 & 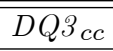 & Dur $_{c c}$ & 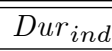 \\
\hline Filtered Historical Simulation & 0.712 & 0.919 & 0.929 & 0.544 & 0.711 & 0.701 & 0.811 & 0.917 & 0.759 & 0.809 & 0.928 & 0.931 & 0.918 & 0.874 & 0.638 \\
\hline Historical Simulation -One Year Data & 0.623 & 0.000 & 0.000 & 0.323 & 0.694 & 0.732 & 0.751 & 0.000 & 0.000 & 0.000 & 0.000 & 0.000 & 0.000 & 0.001 & 0.000 \\
\hline Historical Simulation -Two Year Data & 0.030 & 0.000 & 0.000 & 0.028 & 0.084 & 0.125 & 0.156 & 0.000 & 0.000 & 0.000 & 0.000 & 0.000 & 0.000 & 0.001 & 0.004 \\
\hline Historical Simulation All Sample & 0.932 & 0.000 & 0.000 & 0.510 & 0.950 & 0.954 & 0.933 & 0.000 & 0.000 & 0.000 & 0.000 & 0.000 & 0.000 & 0.000 & 0.000 \\
\hline Riskmetrics & 0.001 & 0.285 & 0.002 & 0.005 & 0.003 & 0.004 & 0.000 & 0.339 & 0.462 & 0.104 & 0.006 & 0.016 & 0.009 & 0.003 & 0.737 \\
\hline Variance-Covariance & 0.030 & 0.000 & 0.000 & 0.028 & 0.084 & 0.138 & 0.179 & 0.000 & 0.000 & 0.000 & 0.000 & 0.000 & 0.000 & 0.000 & 0.000 \\
\hline Extreme Value Theory & 0.000 & 0.038 & 0.000 & 0.000 & 0.000 & 0.000 & 0.000 & 0.002 & 0.000 & 0.000 & 0.000 & 0.000 & 0.000 & 0.000 & 0.090 \\
\hline AR-ARCH1-Normal Distribution & 0.003 & 0.152 & 0.004 & 0.006 & 0.004 & 0.004 & 0.004 & 0.412 & 0.238 & 0.961 & 0.015 & 0.028 & 0.015 & 0.009 & 0.979 \\
\hline AR-GARCH11-Normal Distibution & 0.001 & 0.179 & 0.002 & 0.006 & 0.002 & 0.003 & 0.000 & 0.457 & 0.880 & 0.220 & 0.007 & 0.013 & 0.004 & 0.003 & 0.743 \\
\hline AR-GARCH11-Student Distribution & 0.354 & 0.872 & 0.643 & 0.156 & 0.343 & 0.337 & 0.418 & 0.866 & 0.809 & 0.904 & 0.631 & 0.810 & 0.919 & 0.559 & 0.449 \\
\hline AR-EGARCH11-Normal Distibution & 0.001 & 0.194 & 0.001 & 0.003 & 0.001 & 0.002 & 0.003 & 0.480 & 0.822 & 0.184 & 0.005 & 0.008 & 0.002 & 0.002 & 0.965 \\
\hline AR-EGARCH11-Student Distribution & 0.610 & 0.244 & 0.446 & 0.385 & 0.621 & 0.629 & 0.744 & 0.191 & 0.070 & 0.026 & 0.372 & 0.313 & 0.274 & 0.935 & 0.949 \\
\hline Montecarlo Simulation & 0.610 & 0.972 & 0.878 & 0.149 & 0.607 & 0.595 & 0.694 & 0.971 & 0.689 & 0.804 & 0.875 & 0.888 & 0.975 & 0.716 & 0.463 \\
\hline Combination Mean & 0.064 & 0.079 & 0.039 & 0.161 & 0.066 & 0.087 & 0.086 & 0.268 & 0.575 & 0.099 & 0.112 & 0.051 & 0.014 & 0.146 & 0.972 \\
\hline Combination Median & 0.013 & 0.116 & 0.013 & 0.057 & 0.016 & 0.013 & 0.016 & 0.347 & 0.175 & 0.806 & 0.041 & 0.062 & 0.013 & 0.031 & 0.612 \\
\hline Combination Trim 1 & 0.064 & 0.079 & 0.039 & 0.161 & 0.066 & 0.079 & 0.078 & 0.268 & 0.984 & 0.234 & 0.112 & 0.135 & 0.039 & 0.145 & 0.911 \\
\hline Combination Trim2 & 0.064 & 0.079 & 0.039 & 0.161 & 0.066 & 0.079 & 0.078 & 0.268 & 0.984 & 0.234 & 0.112 & 0.135 & 0.039 & 0.145 & 0.911 \\
\hline Panel B: 1\% & $L R_{u c}$ & $L R_{\text {ind }}$ & $L R_{c c}$ & $L R_{m u c}$ & $D Q 1_{u c}$ & $D Q 2 u c$ & $D Q 3 u c$ & $D Q 1_{\text {ind }}$ & $D Q 2_{i n d}$ & $D Q 3_{\text {ind }}$ & $D Q 1_{c c}$ & $D Q 2 c c$ & $D Q 3 c c$ & $\operatorname{Dur}_{c c}$ & Dur $_{\text {ind }}$ \\
\hline Filtered Historical Simulation & 0.275 & 0.757 & 0.526 & 0.559 & 0.301 & 0.299 & 0.297 & 0.856 & 0.796 & 0.751 & 0.580 & 0.773 & 0.887 & 0.334 & 0.761 \\
\hline Historical Simulation -One Year Data & 0.413 & 0.152 & 0.256 & 0.292 & 0.426 & 0.453 & 0.477 & 0.019 & 0.001 & 0.000 & 0.045 & 0.011 & 0.004 & 0.439 & 0.241 \\
\hline Historical Simulation -Two Year Data & 0.682 & 0.690 & 0.849 & 0.951 & 0.686 & 0.685 & 0.685 & 0.791 & 0.706 & 0.642 & 0.891 & 0.960 & 0.985 & 0.705 & 0.699 \\
\hline Historical Simulation All Sample & 0.599 & 0.594 & 0.756 & 0.768 & 0.585 & 0.579 & 0.637 & 0.683 & 0.560 & 0.007 & 0.795 & 0.889 & 0.000 & 0.327 & 0.139 \\
\hline Riskmetrics & 0.097 & 0.477 & 0.196 & 0.214 & 0.069 & 0.064 & 0.060 & 0.528 & 0.368 & 0.266 & 0.164 & 0.257 & 0.345 & 0.046 & 0.039 \\
\hline Variance-Covariance & 0.007 & 0.058 & 0.004 & 0.021 & 0.005 & 0.015 & 0.023 & 0.000 & 0.000 & 0.000 & 0.000 & 0.000 & 0.000 & 0.000 & 0.002 \\
\hline Extreme Value Theory & 0.097 & 0.477 & 0.196 & 0.197 & 0.069 & 0.064 & 0.118 & 0.528 & 0.368 & 0.297 & 0.164 & 0.257 & 0.509 & 0.342 & 0.619 \\
\hline AR-ARCH1-Normal Distribution & 0.029 & 0.423 & 0.066 & 0.015 & 0.014 & 0.012 & 0.023 & 0.451 & 0.282 & 0.139 & 0.040 & 0.071 & 0.000 & 0.032 & 0.074 \\
\hline AR-ARCH1-Student Distribution & 0.269 & 0.534 & 0.447 & 0.025 & 0.237 & 0.230 & 0.257 & 0.606 & 0.462 & 0.499 & 0.444 & 0.592 & 0.156 & 0.487 & 0.379 \\
\hline AR-GARCH11-Normal Distibution & 0.097 & 0.477 & 0.196 & 0.214 & 0.069 & 0.064 & 0.060 & 0.528 & 0.368 & 0.266 & 0.164 & 0.257 & 0.345 & 0.193 & 0.238 \\
\hline AR-GARCH11-Student Distribution & 0.930 & 0.658 & 0.903 & 0.387 & 0.929 & 0.931 & 0.933 & 0.756 & 0.659 & 0.586 & 0.949 & 0.977 & 0.990 & 0.765 & 0.544 \\
\hline AR-EGARCH11-Normal Distibution & 0.166 & 0.505 & 0.307 & 0.335 & 0.134 & 0.127 & 0.179 & 0.567 & 0.414 & 0.040 & 0.284 & 0.413 & 0.000 & 0.444 & 0.519 \\
\hline AR-EGARCH11-Student Distribution & 0.413 & 0.564 & 0.605 & 0.641 & 0.388 & 0.380 & 0.410 & 0.645 & 0.511 & 0.407 & 0.625 & 0.761 & 0.158 & 0.603 & 0.390 \\
\hline Montecarlo Simulation & 0.413 & 0.564 & 0.605 & 0.641 & 0.388 & 0.380 & 0.372 & 0.645 & 0.511 & 0.417 & 0.625 & 0.761 & 0.845 & 0.821 & 0.727 \\
\hline Combination Mean & 0.413 & 0.564 & 0.605 & 0.587 & 0.388 & 0.380 & 0.450 & 0.645 & 0.511 & 0.013 & 0.625 & 0.761 & 0.000 & 0.568 & 0.354 \\
\hline Combination Median & 0.599 & 0.594 & 0.756 & 0.775 & 0.585 & 0.579 & 0.604 & 0.683 & 0.560 & 0.324 & 0.795 & 0.889 & 0.142 & 0.898 & 0.683 \\
\hline
\end{tabular}

Notes: See Table 2. 
Table A7. Backtesting results TD9

\begin{tabular}{|c|c|c|c|c|c|c|c|c|c|c|c|c|c|c|c|}
\hline Panel A: 5\% & $L R_{u c}$ & $L R_{\text {ind }}$ & $L R_{c c}$ & $L R_{m u c}$ & $D Q 1 u c$ & $\overline{D Q 2 u c}$ & DQ3uc & $D Q 1_{\text {ind }}$ & $D Q 2_{\text {ind }}$ & $D Q 3_{\text {ind }}$ & $D Q 1 c c$ & $D Q 2 c c$ & $D Q 3 c c$ & Dur $_{c c}$ & Dur $_{\text {ind }}$ \\
\hline Filtered Historical Simulation & 0.712 & 0.582 & 0.803 & 0.851 & 0.705 & 0.695 & 0.805 & 0.592 & 0.443 & 0.890 & 0.808 & 0.865 & 0.885 & 0.889 & 0.667 \\
\hline Historical Simulation -One Year Data & 0.431 & 0.000 & 0.000 & 0.665 & 0.503 & 0.527 & 0.627 & 0.000 & 0.000 & 0.000 & 0.000 & 0.000 & 0.000 & 0.001 & 0.000 \\
\hline Historical Simulation - Two Year Data & 0.954 & 0.003 & 0.012 & 0.700 & 0.959 & 0.965 & 0.972 & 0.000 & 0.002 & 0.001 & 0.002 & 0.004 & 0.004 & 0.005 & 0.001 \\
\hline Historical Simulation All Sample & 0.431 & 0.000 & 0.001 & 0.192 & 0.494 & 0.495 & 0.520 & 0.000 & 0.000 & 0.000 & 0.000 & 0.000 & 0.000 & 0.001 & 0.000 \\
\hline Riskmetrics & 0.020 & 0.007 & 0.002 & 0.029 & 0.050 & 0.054 & 0.052 & 0.004 & 0.019 & 0.074 & 0.001 & 0.004 & 0.009 & 0.018 & 0.139 \\
\hline Variance-Covariance & 0.278 & 0.000 & 0.001 & 0.079 & 0.366 & 0.395 & 0.426 & 0.000 & 0.000 & 0.000 & 0.000 & 0.000 & 0.000 & 0.000 & 0.000 \\
\hline Extreme Value Theory & 0.000 & 0.234 & 0.000 & 0.000 & 0.000 & 0.000 & 0.000 & 0.093 & 0.188 & 0.036 & 0.000 & 0.000 & 0.000 & 0.000 & 0.700 \\
\hline AR-ARCH1-Normal Distribution & 0.123 & 0.773 & 0.293 & 0.067 & 0.141 & 0.157 & 0.163 & 0.790 & 0.374 & 0.383 & 0.319 & 0.354 & 0.510 & 0.011 & 0.012 \\
\hline AR-GARCH11-Normal Distibution & 0.217 & 0.877 & 0.461 & 0.027 & 0.232 & 0.226 & 0.181 & 0.886 & 0.762 & 0.913 & 0.481 & 0.620 & 0.712 & 0.293 & 0.430 \\
\hline AR-GARCH11-Student Distribution & 0.002 & 0.826 & 0.006 & 0.004 & 0.001 & 0.001 & 0.002 & 0.795 & 0.445 & 0.798 & 0.003 & 0.007 & 0.010 & 0.013 & 0.905 \\
\hline AR-EGARCH11-Normal Distibution & 0.217 & 0.877 & 0.461 & 0.077 & 0.232 & 0.226 & 0.181 & 0.886 & 0.762 & 0.913 & 0.481 & 0.620 & 0.712 & 0.268 & 0.372 \\
\hline AR-EGARCH11-Student Distribution & 0.002 & 0.826 & 0.006 & 0.004 & 0.001 & 0.000 & 0.002 & 0.795 & 0.249 & 0.784 & 0.003 & 0.004 & 0.003 & 0.010 & 0.521 \\
\hline Montecarlo Simulation & 0.001 & 0.725 & 0.003 & 0.002 & 0.000 & 0.000 & 0.001 & 0.676 & 0.833 & 0.363 & 0.001 & 0.003 & 0.003 & 0.006 & 0.880 \\
\hline Combination Mean & 0.841 & 0.765 & 0.937 & 0.299 & 0.840 & 0.847 & 0.741 & 0.774 & 0.950 & 0.954 & 0.940 & 0.967 & 0.982 & 0.766 & 0.518 \\
\hline Combination Median & 0.523 & 0.914 & 0.811 & 0.345 & 0.528 & 0.540 & 0.454 & 0.919 & 0.735 & 0.785 & 0.816 & 0.865 & 0.916 & 0.385 & 0.251 \\
\hline Combination Trim1 & 0.954 & 0.762 & 0.954 & 0.272 & 0.955 & 0.961 & 0.853 & 0.757 & 0.665 & 0.561 & 0.952 & 0.979 & 0.985 & 0.662 & 0.375 \\
\hline Combination Trim2 & 0.712 & 0.490 & 0.736 & 0.179 & 0.716 & 0.712 & 0.817 & 0.455 & 0.553 & 0.550 & 0.706 & 0.872 & 0.952 & 0.598 & 0.322 \\
\hline Panel B: 1\% & $L R_{u c}$ & $L R_{\text {ind }}$ & $L R_{c c}$ & $L R_{m u c}$ & $D Q 1 u c$ & $D Q 2 u c$ & DQ3uc & $D Q 1_{\text {ind }}$ & $D Q 2_{\text {ind }}$ & $D Q 3_{\text {ind }}$ & $D Q 1_{c c}$ & $D Q 2 c c$ & $D Q 3 c c$ & Dur $_{c c}$ & Dur $_{\text {ind }}$ \\
\hline Filtered Historical Simulation & 0.457 & 0.723 & 0.712 & 0.679 & 0.471 & 0.470 & 0.468 & 0.824 & 0.752 & 0.698 & 0.756 & 0.895 & 0.957 & 0.257 & 0.215 \\
\hline Historical Simulation -One Year Data & 0.269 & 0.181 & 0.221 & 0.406 & 0.273 & 0.263 & 0.256 & 0.029 & 0.250 & 0.493 & 0.046 & 0.087 & 0.148 & 0.313 & 0.198 \\
\hline Historical Simulation - Two Year Data & 0.413 & 0.152 & 0.256 & 0.587 & 0.426 & 0.453 & 0.477 & 0.019 & 0.001 & 0.000 & 0.045 & 0.011 & 0.004 & 0.011 & 0.003 \\
\hline Historical Simulation All Sample & 0.166 & 0.212 & 0.176 & 0.259 & 0.161 & 0.216 & 0.235 & 0.041 & 0.000 & 0.000 & 0.042 & 0.000 & 0.000 & 0.006 & 0.002 \\
\hline Riskmetrics & 0.029 & 0.037 & 0.010 & 0.044 & 0.029 & 0.025 & 0.023 & 0.000 & 0.027 & 0.136 & 0.000 & 0.000 & 0.000 & 0.102 & 0.342 \\
\hline Variance-Covariance & 0.003 & 0.071 & 0.003 & 0.008 & 0.002 & 0.004 & 0.005 & 0.000 & 0.000 & 0.000 & 0.000 & 0.000 & 0.000 & 0.000 & 0.002 \\
\hline Extreme Value Theory & 0.097 & 0.477 & 0.196 & 0.061 & 0.069 & 0.064 & 0.060 & 0.528 & 0.368 & 0.266 & 0.164 & 0.257 & 0.345 & 0.341 & 0.613 \\
\hline AR-ARCH1-Normal Distribution & 0.007 & 0.373 & 0.018 & 0.010 & 0.002 & 0.002 & 0.001 & 0.377 & 0.207 & 0.118 & 0.007 & 0.012 & 0.019 & 0.052 & 0.886 \\
\hline AR-ARCH1-Student Distribution & 0.166 & 0.505 & 0.307 & 0.110 & 0.134 & 0.127 & 0.121 & 0.567 & 0.414 & 0.313 & 0.284 & 0.413 & 0.520 & 0.411 & 0.450 \\
\hline AR-GARCH11-Normal Distibution & 0.054 & 0.450 & 0.118 & 0.031 & 0.032 & 0.029 & 0.027 & 0.490 & 0.324 & 0.222 & 0.086 & 0.143 & 0.202 & 0.253 & 0.861 \\
\hline AR-GARCH11-Student Distribution & 0.097 & 0.477 & 0.196 & 0.061 & 0.069 & 0.064 & 0.060 & 0.528 & 0.368 & 0.266 & 0.164 & 0.257 & 0.345 & 0.341 & 0.613 \\
\hline AR-EGARCH11-Normal Distibution & 0.054 & 0.450 & 0.118 & 0.031 & 0.032 & 0.029 & 0.027 & 0.490 & 0.324 & 0.222 & 0.086 & 0.143 & 0.202 & 0.257 & 0.992 \\
\hline AR-EGARCH11-Student Distribution & 0.682 & 0.690 & 0.849 & 0.714 & 0.686 & 0.685 & 0.685 & 0.791 & 0.706 & 0.642 & 0.891 & 0.960 & 0.985 & 0.538 & 0.406 \\
\hline Montecarlo Simulation & 0.269 & 0.534 & 0.447 & 0.186 & 0.237 & 0.230 & 0.222 & 0.606 & 0.462 & 0.364 & 0.444 & 0.592 & 0.699 & 0.718 & 0.984 \\
\hline Combination Mean & 0.682 & 0.690 & 0.849 & 0.714 & 0.686 & 0.685 & 0.685 & 0.791 & 0.706 & 0.642 & 0.891 & 0.960 & 0.985 & 0.636 & 0.551 \\
\hline Combination Median & 0.930 & 0.658 & 0.903 & 0.664 & 0.929 & 0.931 & 0.933 & 0.756 & 0.659 & 0.586 & 0.949 & 0.977 & 0.990 & 0.797 & 0.591 \\
\hline
\end{tabular}

Notes: See Table 2 
Table A8. Performance Evaluation: All methods

\begin{tabular}{|c|c|c|c|c|c|c|c|c|c|c|c|c|c|c|c|c|c|c|}
\hline \multirow[t]{2}{*}{ Panel A: 5\% } & \multicolumn{3}{|c|}{4 TC CAPE } & \multicolumn{3}{|c|}{4 TC PAN } & \multicolumn{3}{|c|}{ TD3 } & \multicolumn{3}{|c|}{ TD5 } & \multicolumn{3}{|c|}{ TD7 } & \multicolumn{3}{|c|}{ TD9 } \\
\hline & 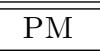 & Ratio & & $\overline{\overline{\mathrm{PM}}}$ & Ratio & & 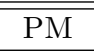 & Ratio & & 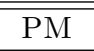 & Ratio & & $\overline{\mathrm{PM}}$ & Ratio & & $\overline{\overline{\mathrm{PM}}}$ & R Ratio & \\
\hline Filtered Historical Simulation & 0.046 & 3.973 & NR & 0.014 & 3.381 & NR & 0.033 & 4.762 & NR & 0.053 & 4.528 & NR & 0.056 & 5.529 & $\mathrm{R}$ & 0.059 & 4.826 & NR \\
\hline Historical Simulation-250 & 0.054 & 4.589 & NR & 0.031 & 7.583 & $\mathrm{R}$ & 0.040 & 5.725 & $\mathrm{R}$ & .062 & 5.268 & NR & 0.062 & 6.150 & NR & 0.072 & 5.970 & $\mathrm{R}$ \\
\hline Historical Simulation-500 & 0.068 & 5.853 & $\mathrm{R}$ & 0.048 & 11.88 & $\mathrm{R}$ & .046 & 6.551 & $\mathrm{R}$ & .064 & 5.444 & NR & 0.056 & 5.542 & NR & 0.078 & 6.416 & $\mathrm{R}$ \\
\hline Historical SimulationAll & 0.183 & 15.66 & $\mathrm{R}$ & 0.056 & 13.82 & $\mathrm{R}$ & .045 & 6.399 & $\mathrm{R}$ & .075 & 6.444 & $\mathrm{R}$ & 0.080 & 7.925 & $\mathrm{R}$ & 0.080 & 6.563 & $\mathrm{R}$ \\
\hline Riskmetrics & 0.048 & 4.104 & NR & 0.021 & 5.241 & NR & .038 & 5.429 & NR & .052 & 4.461 & NR & 0.038 & 3.739 & NR & 0.044 & 3.588 & NR \\
\hline Variance-Covariance & 0.162 & 13.92 & $\mathrm{R}$ & 0.048 & 11.86 & $\mathrm{R}$ & .046 & 6.611 & $\mathrm{R}$ & .066 & 5.631 & NR & 0.066 & 6.562 & $\mathrm{R}$ & 0.075 & 6.181 & $\mathrm{R}$ \\
\hline Extreme Value Theory & 0.069 & 5.907 & $\mathrm{R}$ & 0.018 & 4.478 & NR & .064 & 9.047 & $\mathrm{R}$ & .108 & 9.237 & $\mathrm{R}$ & 0.108 & 10.720 & $\mathrm{R}$ & 0.111 & 9.121 & $\mathrm{R}$ \\
\hline $\operatorname{AR}-\operatorname{ARCH}(1)-\mathrm{N}$ & 0.071 & 6.044 & $\mathrm{R}$ & 0.018 & 4.548 & NR & .036 & 5.081 & NR & .063 & 5.391 & $\mathrm{NR}$ & 0.046 & 4.580 & NR & 0.057 & 4.686 & NR \\
\hline $\operatorname{AR}-\operatorname{ARCH}(1)-\mathrm{t}$ & 0.08 & 6.87 & $\mathrm{R}$ & 0.023 & 5.618 & $\mathrm{R}$ & .039 & 5.566 & $\mathrm{R}$ & .083 & 7.073 & $\mathrm{R}$ & 0.063 & 6.251 & $\mathrm{R}$ & 0.084 & 6.885 & $\mathrm{R}$ \\
\hline AR-GARCH(1.1)-N & 0.043 & 3.649 & NR & 0.013 & 3.133 & NR & 35 & 4.998 & NR & 60 & 5.134 & NR & 0.039 & 3.910 & NR & .051 & 4.226 & NR \\
\hline$A R-$ & 044 & 3.731 & NR & 0.013 & 3.312 & NR & 039 & 5.590 & $\mathrm{R}$ & .069 & 5.917 & $\mathrm{R}$ & 0.057 & 5.692 & $\mathrm{R}$ & 0.077 & .373 & $\mathrm{R}$ \\
\hline AR-EGARCH(1.1)-N & 0.042 & 3.616 & NR & 0.012 & 3.058 & NR & .034 & 4.871 & NR & .056 & 4.816 & NR & 0.040 & 4.011 & NR & 0.050 & 4.107 & NR \\
\hline $\operatorname{AR}-\operatorname{EGARCH}(1.1)-\mathrm{t}$ & 0.045 & 3.887 & NR & 0.012 & 3.074 & NR & .035 & 4.919 & NR & .055 & 4.734 & NR & 0.056 & 5.585 & $\mathrm{R}$ & 0.074 & 6.119 & $\mathrm{R}$ \\
\hline Monte Carlo Sim & 0.044 & 3.778 & NR & 0.013 & 3.277 & NR & 037 & 5.245 & $\mathrm{NR}$ & & 5.664 & $\mathrm{R}$ & 0.055 & 5.463 & $\mathrm{R}$ & 0.080 & 6.555 & $\mathrm{R}$ \\
\hline Com & 041 & 3.553 & NR & 0.018 & 4.407 & NR & & & NR & & 5.067 & NR & 0.046 & 4.558 & NR & 0.055 & 4.549 & NR \\
\hline Coml & 043 & 3.678 & NR & 0.012 & 3.063 & NR & 034 & 4.863 & NR & 56 & 4.819 & NR & 0.044 & 4.321 & NR & 0.054 & 4.485 & NR \\
\hline Comb & 042 & 3.564 & NR & 0.017 & 4.246 & NR & 034 & 4.784 & NR & .061 & 5.177 & NR & 0.048 & 4.731 & NR & 0.056 & 4.599 & NR \\
\hline Combination Trim2 & 042 & 3.622 & NR & 0.016 & 4.009 & & & 4.759 & & & 5.197 & $\mathrm{NR}$ & 0.048 & 4.732 & NR & & 4.753 & NR \\
\hline \multirow[t]{2}{*}{ Panel B: 1\% } & \multicolumn{3}{|c|}{4 TC CAPE } & \multicolumn{3}{|c|}{4 TC PAN } & \multicolumn{3}{|c|}{ TD3 } & \multicolumn{3}{|c|}{ TD5 } & \multicolumn{3}{|c|}{ TD7 } & \multicolumn{3}{|c|}{ TD9 } \\
\hline & $\overline{\mathrm{PM}}$ & Ratio & & $\overline{\mathrm{PM}}$ & Ratio & & PM & Ratio & & $\overline{\mathrm{PM}}$ & Ratio & & $\overline{\mathrm{PM}}$ & Ratio & & $\overline{\mathrm{PM}}$ & Ratio & \\
\hline 110 & 0.007 & 2.580 & 10 & 0.002 & 1.989 & 1 & 0.004 & 3.949 & $\mathrm{~N}$ & 0.006 & 3.254 & $\mathrm{NR}$ & 0.005 & 3.400 & NR & 0.006 & 3.561 & NR \\
\hline Histc & 0.015 & 5.759 & NR & 0.005 & & NR & & & NR & & 7.028 & $\mathrm{NR}$ & 0.010 & 6.592 & $\mathrm{R}$ & 0.010 & 5.380 & NR \\
\hline Histc & 0.012 & 4.546 & NR & 0.007 & 7.843 & $\mathrm{R}$ & 0.006 & 5.262 & NR & 012 & 7.047 & NR & 0.006 & 4.041 & NR & 0.017 & 9.689 & $\mathrm{R}$ \\
\hline Historical Simu & 0.040 & 15.973 & $\mathrm{R}$ & 0.012 & 13.714 & $\mathrm{R}$ & 0.005 & 4.915 & NR & 0.012 & 6.662 & NR & 0.008 & 5.335 & NR & 0.018 & 10.253 & $\mathrm{R}$ \\
\hline Riskmetrics & 0.014 & 5.578 & NR & 0.004 & 4.078 & NR & 0.007 & 5.976 & $D$ & 0.010 & 5.440 & $\mathrm{R}$ & 0.007 & 4.809 & NR & 0.010 & 5.482 & NR \\
\hline Variance-Co & 0.050 & 19.601 & $\mathrm{R}$ & 0.027 & 31.344 & $\mathrm{R}$ & & 6.623 & $\mathrm{R}$ & & 12.178 & $\kappa$ & 0.022 & 14.986 & $\mathrm{R}$ & 0.024 & 13.603 & $\mathrm{R}$ \\
\hline Extreme Value & 0.009 & 3.453 & NR & 0.002 & 2.038 & NR & 0.006 & 5.036 & $\mathrm{R}$ & 0.005 & 2.872 & NR & 0.005 & 3.528 & NR & 0.005 & 2.859 & NR \\
\hline AR-ARCH(1)-N & 0.026 & 10.206 & $\mathrm{R}$ & 0.005 & 5.247 & NR & 0.009 & 8.358 & $\mathrm{R}$ & 0.019 & 10.710 & $\mathrm{R}$ & 0.013 & 9.009 & $\mathrm{R}$ & 0.012 & 6.801 & $\mathrm{R}$ \\
\hline $\operatorname{AR}-\operatorname{ARCH}(1)-\mathrm{t}$ & 0.011 & 4.266 & NR & 0.003 & 3.516 & NR & 0.004 & 3.998 & NR & 0.008 & 4.536 & $\mathrm{NR}$ & 0.008 & 5.742 & NR & 0.006 & 3.527 & NR \\
\hline AR-GARCH $(1.1$ & 0.011 & 4.259 & NR & 0.002 & 2.644 & NR & 0.007 & 6.765 & $\mathrm{R}$ & 0.017 & 9.810 & $\mathrm{R}$ & 0.008 & 5.481 & NR & 0.011 & 5.970 & $\mathrm{R}$ \\
\hline $\mathrm{AR}$ & 0.006 & 2.497 & NR & 0.002 & 1.946 & NR & 0.006 & 5.772 & $\mathrm{R}$ & 0.005 & 3.094 & NR & 0.005 & 3.689 & NR & 0.006 & 3.660 & NR \\
\hline AR-EGARCH(1.1)-N & 0.011 & 4.486 & NR & 0.002 & 2.530 & NR & 0.009 & 8.565 & $\mathrm{R}$ & 0.013 & 7.476 & $\mathrm{R}$ & 0.009 & 6.201 & $\mathrm{R}$ & 0.011 & 6.022 & $\mathrm{R}$ \\
\hline $\operatorname{AR-EGARCH}(1.1)-\mathrm{t}$ & 0.007 & 2.593 & NR & 0.002 & 1.905 & NR & 0.006 & 5.392 & $\mathrm{R}$ & 0.005 & 2.935 & NR & 0.006 & 4.420 & NR & 0.005 & 2.828 & NR \\
\hline Monte Carlo Simulation & 0.006 & 2.446 & NR & 0.002 & 1.932 & NR & 0.006 & 5.808 & $\mathrm{R}$ & 0.006 & 3.401 & NR & 0.006 & 4.418 & NR & 0.006 & 3.522 & NR \\
\hline Combination Mean & 0.008 & 3.104 & NR & 0.004 & 4.426 & NR & & 4.452 & NR & & 3.411 & NR & 0.007 & 4.936 & NR & 0.007 & 4.232 & NR \\
\hline Combination Median & 0.008 & 3.147 & NR & 0.002 & 2.096 & NR & 0.005 & 4.849 & NR & 0.006 & 3.553 & NR & 0.007 & 4.735 & NR & 0.008 & 4.466 & NR \\
\hline Combination Trim1 & 0.006 & 2.333 & NR & 0.003 & 3.376 & NR & 0.005 & 4.403 & NR & 0.005 & 3.135 & NR & 0.005 & 3.739 & NR & 0.007 & 3.948 & NR \\
\hline Combination Trim2 & 0.008 & 3.172 & NR & 0.003 & 3.745 & NR & 0.005 & 4.477 & NR & 0.006 & 3.460 & $\mathrm{NR}$ & 0.007 & 4.939 & NR & 0.007 & 4.197 & NR \\
\hline
\end{tabular}

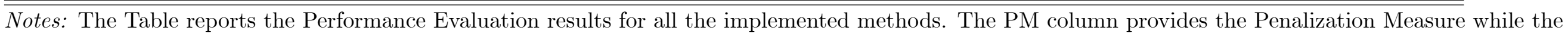
Ratio column provides the corresponding ratio. $R(N R)$ suggests rejection (non-rejection) with respect to the performance equality test. 
Table A9. Performance Evaluation: Second Stage

\begin{tabular}{|c|c|c|c|c|c|c|c|c|c|c|c|c|c|c|c|c|c|c|}
\hline \multirow[t]{2}{*}{ Panel A: 5\% } & \multicolumn{3}{|c|}{4 TC CAPE } & \multicolumn{3}{|c|}{4 4 TC PAN } & \multicolumn{3}{|c|}{ TD3 } & \multicolumn{3}{|c|}{ TD5 } & \multicolumn{3}{|c|}{ TD7 } & \multicolumn{3}{|c|}{ TD9 } \\
\hline & $\overline{\mathrm{PM}}$ & Ratio & & $\overline{\mathrm{PM}}$ & Ratio & & $\overline{\mathrm{PM}}$ & Ratio & & $\overline{\mathrm{PM}}$ & Ratio & & $\overline{\mathrm{PM}}$ & Ratio & & $\overline{\mathrm{PM}}$ & Ratio & \\
\hline Filtered Historical Simulation & 0.046 & 7.700 & $\mathrm{R}$ & 0.014 & 6.869 & NR & 0.033 & 6.670 & NR & 0.053 & 6.348 & $\mathrm{R}$ & 0.056 & 8.067 & $\mathrm{R}$ & 0.059 & 6.809 & NR \\
\hline Historical Simulation-250 & 0.054 & 8.895 & $\mathrm{R}$ & - & - & - & 0.040 & 8.019 & $\mathrm{R}$ & 0.062 & 7.386 & $\mathrm{R}$ & 0.062 & 8.972 & $\mathrm{R}$ & 0.072 & 8.422 & $\mathrm{R}$ \\
\hline Historical Simulation-500 & 0.068 & 11.343 & $\mathrm{R}$ & - & - & - & - & - & - & 0.064 & 7.632 & $\mathrm{R}$ & 0.056 & 8.085 & $\mathrm{R}$ & 0.078 & 9.052 & $\mathrm{R}$ \\
\hline Historical SimulationAll & - & - & - & - & - & - & - & - & - & - & - & - & - & - & - & - & - & - \\
\hline Riskmetrics & 0.048 & 7.953 & NR & 0.021 & 10.646 & $\mathrm{R}$ & 0.038 & 7.605 & $\mathrm{R}$ & 0.052 & 6.253 & NR & 0.038 & 5.455 & NR & 0.044 & 5.062 & NR \\
\hline Variance-Covariance & - & - & - & - & - & - & - & - & - & 0.066 & 7.895 & $\mathrm{R}$ & - & - & - & 0.075 & 8.721 & $\mathrm{R}$ \\
\hline Extreme Value Theory & - & - & - & 0.018 & 9.097 & $\mathrm{R}$ & - & - & - & - & - & - & - & - & - & - & - & - \\
\hline AR-ARCH(1)-N & - & - & - & 0.018 & 9.238 & $\mathrm{R}$ & 0.036 & 7.117 & NR & 0.063 & 7.557 & $\mathrm{R}$ & 0.046 & 6.682 & NR & 0.057 & 6.611 & NR \\
\hline $\operatorname{AR}-\operatorname{ARCH}(1)-\mathrm{t}$ & - & - & - & - & - & - & 0.039 & 7.796 & $\mathrm{R}$ & - & - & - & - & - & - & - & - & - \\
\hline AR-GARCH(1.1)-N & 0.043 & 7.072 & NR & 0.013 & 6.365 & NR & 0.035 & 7.000 & NR & 0.060 & 7.197 & NR & 0.039 & 5.704 & NR & 0.051 & 5.963 & NR \\
\hline AR-GARCH(1.1)-t & 0.044 & 7.230 & $\mathrm{NR}$ & 0.013 & 6.727 & NR & 0.039 & 7.830 & $\mathrm{R}$ & - & - & - & 0.057 & 8.305 & $\mathrm{R}$ & 0.077 & 8.991 & $\mathrm{R}$ \\
\hline AR-EGARCH(1.1)-N & 0.042 & 7.009 & NR & 0.012 & 6.211 & NR & 0.034 & 6.823 & NR & 0.056 & 6.751 & NR & 0.040 & 5.851 & NR & 0.050 & 5.794 & NR \\
\hline $\operatorname{AR}-\operatorname{EGARCH}(1.1)-\mathrm{t}$ & 0.045 & 7.533 & NR & 0.012 & 6.245 & NR & 0.035 & 6.891 & $\mathrm{NR}$ & 0.055 & 6.637 & $\mathrm{R}$ & 0.056 & 8.148 & $\mathrm{R}$ & 0.074 & 8.634 & $\mathrm{R}$ \\
\hline Monte Carlo Simulation & 0.044 & 7.323 & NR & 0.013 & 6.656 & NR & 0.037 & 7.346 & $\mathrm{R}$ & 0.066 & 7.941 & $\mathrm{R}$ & 0.055 & 7.971 & $\mathrm{R}$ & - & - & - \\
\hline Combination Mean & 0.041 & 6.887 & NR & 0.018 & 8.953 & $\mathrm{R}$ & 0.034 & 6.725 & NR & 0.059 & 7.103 & NR & 0.046 & 6.650 & NR & 0.055 & 6.418 & NR \\
\hline Combination Median & 0.043 & 7.128 & $\mathrm{NR}$ & 0.012 & 6.222 & NR & 0.034 & 6.811 & $\mathrm{NR}$ & 0.056 & 6.755 & NR & 0.044 & 6.304 & NR & 0.054 & 6.327 & NR \\
\hline Combination Trim1 & 0.042 & 6.907 & NR & 0.017 & 8.625 & $\mathrm{R}$ & 0.034 & 6.701 & NR & 0.061 & 7.258 & $\mathrm{R}$ & 0.048 & 6.902 & NR & 0.056 & 6.489 & NR \\
\hline Combination Trim2 & 0.042 & 7.020 & NR & 0.016 & 8.145 & $\mathrm{R}$ & 0.033 & 6.666 & NR & 0.061 & 7.286 & $\mathrm{R}$ & 0.048 & 6.904 & NR & 0.058 & 6.707 & NR \\
\hline \multirow[t]{2}{*}{ Panel B: 1\% } & \multicolumn{3}{|c|}{4 TC CAPE } & \multicolumn{3}{|c|}{4 TC PAN } & \multicolumn{3}{|c|}{ TD3 } & \multicolumn{3}{|c|}{ TD5 } & \multicolumn{3}{|c|}{ TD7 } & \multicolumn{3}{|c|}{ TD9 } \\
\hline & PM & Ratio & & PM & Ratio & & PM & Ratio & & PM & Ratio & & $\mathrm{PM}$ & Ratio & & PM & Ratio & \\
\hline Filtered Historical Simulation & 0.007 & 5.305 & NR & 0.002 & 4.223 & $\mathrm{NR}$ & 0.004 & 5.666 & NR & 0.006 & 5.438 & NR & 0.005 & 5.379 & NR & 0.006 & 5.970 & NR \\
\hline Historical Simulation-250 & 0.015 & 11.840 & $\mathrm{R}$ & 0.005 & 11.956 & $\mathrm{R}$ & 0.006 & 7.745 & $\mathrm{R}$ & 0.012 & 11.747 & $\mathrm{R}$ & - & - & - & 0.010 & 9.018 & $\mathrm{R}$ \\
\hline Historical Simulation-500 & 0.012 & 9.347 & $\mathrm{R}$ & - & - & - & 0.006 & 7.551 & $\mathrm{R}$ & 0.012 & 11.778 & NR & 0.006 & 6.393 & NR & - & - & - \\
\hline Historical SimulationAll & - & - & - & - & - & - & 0.005 & 7.053 & NR & 0.012 & 11.136 & NR & 0.008 & 8.441 & $\mathrm{R}$ & - & - & - \\
\hline Riskmetrics & - & - & - & 0.004 & 8.657 & $\mathrm{R}$ & 0.007 & 8.576 & $\mathrm{R}$ & 0.010 & 9.093 & $\mathrm{R}$ & 0.007 & 7.608 & $\mathrm{R}$ & 0.010 & 9.189 & $\mathrm{R}$ \\
\hline Variance-Covariance & - & - & - & - & - & - & - & - & - & - & - & - & - & - & - & - & - & - \\
\hline Extreme Value Theory & 0.009 & 7.098 & $\mathrm{R}$ & 0.002 & 4.328 & NR & 0.006 & 7.227 & $\mathrm{R}$ & 0.005 & 4.801 & NR & 0.005 & 5.580 & NR & 0.005 & 4.793 & NR \\
\hline AR-ARCH(1)-N & - & - & - & 0.005 & 11.139 & $\mathrm{R}$ & - & - & - & - & - & - & - & - & - & - & - & - \\
\hline $\mathrm{AR}-\mathrm{ARCH}(1)-\mathrm{t}$ & 0.011 & 8.770 & $\mathrm{R}$ & 0.003 & 7.466 & $\mathrm{R}$ & 0.004 & 5.737 & NR & 0.008 & 7.582 & $\mathrm{R}$ & 0.008 & 9.084 & $\mathrm{R}$ & 0.006 & 5.913 & NR \\
\hline AR-GARCH(1.1)-N & 0.011 & 8.755 & $\mathrm{R}$ & 0.002 & 5.614 & NR & - & - & - & - & - & - & 0.008 & 8.671 & $\mathrm{R}$ & 0.011 & 10.008 & $\mathrm{R}$ \\
\hline AR-GARCH(1.1)-t & 0.006 & 5.134 & NR & 0.002 & 4.133 & NR & 0.006 & 8.282 & $\mathrm{R}$ & 0.005 & 5.171 & NR & 0.005 & 5.836 & NR & 0.006 & 6.136 & NR \\
\hline AR-EGARCH(1.1)-N & 0.011 & 9.223 & $\mathrm{R}$ & 0.002 & 5.371 & NR & - & - & - & - & - & - & - & - & - & 0.011 & 10.095 & $\mathrm{R}$ \\
\hline AR-EGARCH(1.1)-t & 0.007 & 5.331 & NR & 0.002 & 4.045 & NR & 0.006 & 7.737 & $\mathrm{R}$ & 0.005 & 4.906 & NR & 0.006 & 6.993 & $\mathrm{R}$ & 0.005 & 4.740 & NR \\
\hline Monte Carlo Simulation & 0.006 & 5.028 & NR & 0.002 & 4.102 & NR & 0.006 & 8.335 & $\mathrm{R}$ & 0.006 & 5.685 & NR & 0.006 & 6.990 & NR & 0.006 & 5.904 & NR \\
\hline Combination Mean & 0.008 & 6.381 & NR & 0.004 & 9.397 & $\mathrm{R}$ & 0.005 & 6.388 & $\mathrm{NR}$ & 0.006 & 5.701 & NR & 0.007 & 7.808 & $\mathrm{R}$ & 0.007 & 7.094 & NR \\
\hline Combination Median & 0.008 & 6.469 & NR & 0.002 & 4.450 & NR & 0.005 & 6.958 & NR & 0.006 & 5.939 & NR & 0.007 & 7.490 & $\mathrm{R}$ & 0.008 & 7.486 & $\mathrm{R}$ \\
\hline Combination Trim 1 & 0.006 & 4.797 & $\mathrm{NR}$ & 0.003 & 7.168 & $\mathrm{R}$ & 0.005 & 6.319 & $\mathrm{NR}$ & 0.005 & 5.239 & NR & 0.005 & 5.915 & NR & 0.007 & 6.617 & NR \\
\hline Combination Trim2 & 0.008 & 6.521 & NR & 0.003 & 7.952 & $\mathrm{R}$ & 0.005 & 6.425 & NR & 0.006 & 5.784 & NR & 0.007 & 7.813 & $\mathrm{R}$ & 0.007 & 7.036 & NR \\
\hline
\end{tabular}

Notes: The Table reports the second stage Performance Evaluation results. For each index the worst performing methods are excluded and the analysis is repeated with the remaining methods. The PM column provides the Penalization Measure while the Ratio column provides the corresponding ratio. $R(N R)$ suggests rejection (non-rejection) with respect to the performance equality test. 


\begin{tabular}{|c|c|c|c|c|c|c|}
\hline & 4 TC CAPE & & 4 TC PAN & & TD3 & \\
\hline 1 & AR-EGARCH11-Normal Distibution & NR & "AR-GARCH11-Normal Distibution & NR & "AR-EGARCH11-Student Distribution & 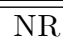 \\
\hline 2 & AR-GARCH11-Normal Distibution & NR & Montecarlo Simulation & NR & Montecarlo Simulation & $\mathrm{R}$ \\
\hline 3 & Combination Median & NR & AR-GARCH11-Student Distribution & NR & AR-ARCH1-Student Distribution & $\mathrm{R}$ \\
\hline 4 & AR-GARCH11-Student Distribution & NR & Filtered Historical Simulation & NR & AR-GARCH11-Student Distribution & $\mathrm{R}$ \\
\hline 5 & Montecarlo Simulation & NR & - & & - & \\
\hline 6 & AR-EGARCH11-Student Distribution & NR & - & & - & \\
\hline 7 & - & & - & & - & \\
\hline 8 & - & & - & & - & \\
\hline 9 & - & & - & & - & \\
\hline 10 & - & & - & & - & \\
\hline 11 & - & & - & & - & \\
\hline 12 & - & & - & & - & \\
\hline 13 & - & & - & & - & \\
\hline 14 & - & & - & & - & \\
\hline \multicolumn{7}{|c|}{ Panel B: 1\% } \\
\hline & 4 TC CAPE & & 4 TC PAN & & TD3 & \\
\hline 1 & Combination Trim1 & NR & AR-EGARCH11-Student Distribution & NR & Filtered Historical Simulation & NR \\
\hline 2 & Montecarlo Simulation & NR & Montecarlo Simulation & NR & AR-ARCH1-Student Distribution & NR \\
\hline 3 & AR-GARCH11-Student Distribution & NR & AR-GARCH11-Student Distribution & NR & Combination Trim 1 & NR \\
\hline 4 & Combination Mean & NR & Filtered Historical Simulation & NR & Combination Mean & NR \\
\hline 5 & Combination Trim2 & NR & Combination Median & NR & Combination Trim2 & NR \\
\hline 6 & - & & AR-EGARCH11-Normal Distibution & NR & Combination Median & NR \\
\hline 7 & - & & AR-GARCH11-Normal Distibution & NR & Extreme Value Theory & $\mathrm{R}$ \\
\hline 8 & - & & AR-ARCH1-Student Distribution & $\mathrm{R}$ & Historical Simulation -Two Year Data & $\mathrm{R}$ \\
\hline 9 & - & & Combination Trim2 & $\mathrm{R}$ & AR-EGARCH11-Student Distribution & $\mathrm{R}$ \\
\hline 10 & - & & Combination Mean & $\mathrm{R}$ & AR-GARCH11-Student Distribution & $\mathrm{R}$ \\
\hline 11 & - & & Historical Simulation -One Year Data & $\mathrm{R}$ & Montecarlo Simulation & $\mathrm{R}$ \\
\hline 12 & - & & - & & - & \\
\hline 13 & - & & - & & - & \\
\hline 14 & - & & - & & - & \\
\hline
\end{tabular}

Notes: The Table reports the ranking of the statistically accepted methods according to the PM measure presented in Table $9 . R(N R)$ suggests rejection (non-rejection) with respect to the performance equality test. 


\begin{tabular}{|c|c|c|c|c|c|c|}
\hline \multicolumn{7}{|c|}{ Panel A: 5\% } \\
\hline & $\overline{\text { TD5 }}$ & & $\overline{\text { TD7 }}$ & & $\overline{\text { TD9 }}$ & \\
\hline$\overline{1} 1$ & Riskmetrics & NR & Combination Median & NR & "AR-EGARCH11-Normal Distibution & NR \\
\hline 2 & Filtered Historical Simulation & $\mathrm{R}$ & Combination Mean & NR & AR-GARCH11-Normal Distibution & NR \\
\hline 3 & AR-EGARCH11-Student Distribution & $\mathrm{R}$ & Combination Trim 1 & NR & Combination Median & NR \\
\hline 4 & AR-EGARCH11-Normal Distibution & NR & Combination Trim2 & NR & Combination Mean & NR \\
\hline 5 & Combination Median & NR & Montecarlo Simulation & $\mathrm{R}$ & Combination Trim1 & NR \\
\hline 6 & Combination Mean & NR & Filtered Historical Simulation & $\mathrm{R}$ & AR-ARCH1-Normal Distribution & NR \\
\hline 7 & AR-GARCH11-Normal Distibution & NR & AR-EGARCH11-Student Distribution & $\mathrm{R}$ & Combination Trim2 & $\mathrm{NR}$ \\
\hline 8 & Combination Trim1 & $\mathrm{R}$ & AR-GARCH11-Student Distribution & $\mathrm{R}$ & Filtered Historical Simulation & NR \\
\hline 9 & Combination Trim2 & $\mathrm{R}$ & - & & - & \\
\hline 10 & Historical Simulation -One Year Data & $\mathrm{R}$ & - & & - & \\
\hline 11 & AR-ARCH1-Normal Distribution & $\mathrm{R}$ & - & & - & \\
\hline 12 & Historical Simulation -Two Year Data & $\mathrm{R}$ & - & & - & \\
\hline 13 & Variance-Covariance & $\mathrm{R}$ & - & & - & \\
\hline 14 & - & & - & & - & \\
\hline \multicolumn{7}{|c|}{ Panel B: 1\% } \\
\hline & TD5 & & TD7 & & TD9 & \\
\hline 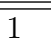 & Extreme Value Theory & $\overline{\mathrm{NR}}$ & Filtered Historical Simulation & $\overline{\mathrm{NR}}$ & " AR-EGARCH11-Student Distribution & $\overline{\mathrm{NR}}$ \\
\hline 2 & AR-EGARCH11-Student Distribution & NR & Extreme Value Theory & NR & Extreme Value Theory & NR \\
\hline 3 & AR-GARCH11-Student Distribution & NR & AR-GARCH11-Student Distribution & NR & Montecarlo Simulation & NR \\
\hline 4 & Combination Trim1 & NR & Combination Trim1 & NR & AR-ARCH1-Student Distribution & NR \\
\hline 5 & Filtered Historical Simulation & NR & Historical Simulation -Two Year Data & NR & Filtered Historical Simulation & NR \\
\hline 6 & Montecarlo Simulation & NR & Montecarlo Simulation & NR & AR-GARCH11-Student Distribution & NR \\
\hline 7 & Combination Mean & NR & AR-EGARCH11-Student Distribution & $\mathrm{R}$ & Combination Trim 1 & NR \\
\hline 8 & Combination Trim2 & NR & Combination Median & $\mathrm{R}$ & Combination Trim2 & NR \\
\hline 9 & Combination Median & NR & Riskmetrics & $\mathrm{R}$ & Combination Mean & NR \\
\hline 10 & AR-ARCH1-Student Distribution & $\mathrm{R}$ & Combination Mean & $\mathrm{R}$ & Combination Median & $\mathrm{R}$ \\
\hline 11 & Riskmetrics & $\mathrm{R}$ & Combination Trim2 & $\mathrm{R}$ & AR-GARCH11-Normal Distibution & $\mathrm{R}$ \\
\hline 12 & Historical Simulation All Sample & NR & Historical Simulation All Sample & $\mathrm{R}$ & AR-EGARCH11-Normal Distibution & $\mathrm{R}$ \\
\hline 13 & Historical Simulation -One Year Data & $\mathrm{R}$ & AR-GARCH11-Normal Distibution & $\mathrm{R}$ & - & \\
\hline 14 & Historical Simulation -Two Year Data & NR & AR-ARCH1-Student Distribution & $\mathrm{R}$ & - & \\
\hline
\end{tabular}

Notes: The Table reports the ranking of the statistically accepted methods according to the PM measure presented in Table $9 . R(N R)$ suggests rejection (non-rejection) with respect to the performance equality test. 


\begin{tabular}{|c|c|c|c|c|c|c|}
\hline \multicolumn{7}{|c|}{ Panel A: 5\% } \\
\hline & $\overline{\text { TD5 }}$ & & $\overline{\text { TD7 }}$ & & $\overline{\text { TD9 }}$ & \\
\hline$\overline{1} 1$ & Riskmetrics & NR & Combination Median & NR & "AR-EGARCH11-Normal Distibution & NR \\
\hline 2 & Filtered Historical Simulation & $\mathrm{R}$ & Combination Mean & NR & AR-GARCH11-Normal Distibution & NR \\
\hline 3 & AR-EGARCH11-Student Distribution & $\mathrm{R}$ & Combination Trim 1 & NR & Combination Median & NR \\
\hline 4 & AR-EGARCH11-Normal Distibution & NR & Combination Trim2 & NR & Combination Mean & NR \\
\hline 5 & Combination Median & NR & Montecarlo Simulation & $\mathrm{R}$ & Combination Trim1 & NR \\
\hline 6 & Combination Mean & NR & Filtered Historical Simulation & $\mathrm{R}$ & AR-ARCH1-Normal Distribution & NR \\
\hline 7 & AR-GARCH11-Normal Distibution & NR & AR-EGARCH11-Student Distribution & $\mathrm{R}$ & Combination Trim2 & $\mathrm{NR}$ \\
\hline 8 & Combination Trim1 & $\mathrm{R}$ & AR-GARCH11-Student Distribution & $\mathrm{R}$ & Filtered Historical Simulation & NR \\
\hline 9 & Combination Trim2 & $\mathrm{R}$ & - & & - & \\
\hline 10 & Historical Simulation -One Year Data & $\mathrm{R}$ & - & & - & \\
\hline 11 & AR-ARCH1-Normal Distribution & $\mathrm{R}$ & - & & - & \\
\hline 12 & Historical Simulation -Two Year Data & $\mathrm{R}$ & - & & - & \\
\hline 13 & Variance-Covariance & $\mathrm{R}$ & - & & - & \\
\hline 14 & - & & - & & - & \\
\hline \multicolumn{7}{|c|}{ Panel B: 1\% } \\
\hline & TD5 & & TD7 & & TD9 & \\
\hline  & Extreme Value Theory & $\overline{\mathrm{NR}}$ & Filtered Historical Simulation & $\overline{\mathrm{NR}}$ & " AR-EGARCH11-Student Distribution & $\overline{\mathrm{NR}}$ \\
\hline 2 & AR-EGARCH11-Student Distribution & NR & Extreme Value Theory & NR & Extreme Value Theory & NR \\
\hline 3 & AR-GARCH11-Student Distribution & NR & AR-GARCH11-Student Distribution & NR & Montecarlo Simulation & NR \\
\hline 4 & Combination Trim1 & NR & Combination Trim1 & NR & AR-ARCH1-Student Distribution & NR \\
\hline 5 & Filtered Historical Simulation & NR & Historical Simulation -Two Year Data & NR & Filtered Historical Simulation & NR \\
\hline 6 & Montecarlo Simulation & NR & Montecarlo Simulation & NR & AR-GARCH11-Student Distribution & NR \\
\hline 7 & Combination Mean & NR & AR-EGARCH11-Student Distribution & $\mathrm{R}$ & Combination Trim 1 & NR \\
\hline 8 & Combination Trim2 & NR & Combination Median & $\mathrm{R}$ & Combination Trim2 & NR \\
\hline 9 & Combination Median & NR & Riskmetrics & $\mathrm{R}$ & Combination Mean & NR \\
\hline 10 & AR-ARCH1-Student Distribution & $\mathrm{R}$ & Combination Mean & $\mathrm{R}$ & Combination Median & $\mathrm{R}$ \\
\hline 11 & Riskmetrics & $\mathrm{R}$ & Combination Trim2 & $\mathrm{R}$ & AR-GARCH11-Normal Distibution & $\mathrm{R}$ \\
\hline 12 & Historical Simulation All Sample & NR & Historical Simulation All Sample & $\mathrm{R}$ & AR-EGARCH11-Normal Distibution & $\mathrm{R}$ \\
\hline 13 & Historical Simulation -One Year Data & $\mathrm{R}$ & AR-GARCH11-Normal Distibution & $\mathrm{R}$ & - & \\
\hline 14 & Historical Simulation -Two Year Data & NR & AR-ARCH1-Student Distribution & $\mathrm{R}$ & - & \\
\hline
\end{tabular}

Notes: The Table reports the ranking of the statistically accepted methods according to the PM measure presented in Table $9 . R(N R)$ suggests rejection (non-rejection) with respect to the performance equality test. 